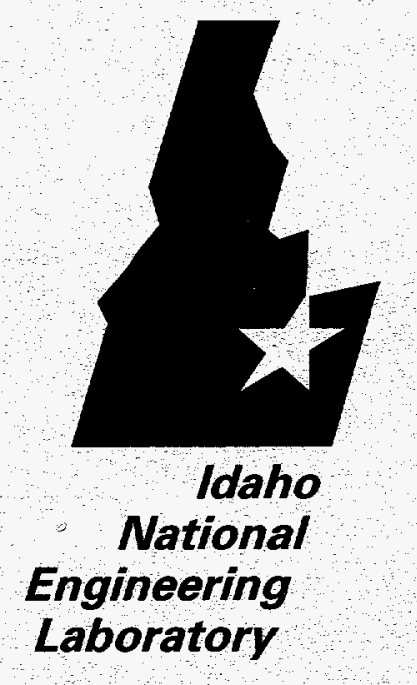

INEL-96/0343

October 1996

\title{
Uncertainty Analysis of the SWEPP PAN Assay System for Glass Waste (Content Codes 440, 441 and 442)
}

\section{areser \\ DEC 191900 \\ OSTI}

\author{
Larry G. Blackwood \\ Yale D. Harker \\ Teresa R. Meachum \\ Woo Y. Yoon
}

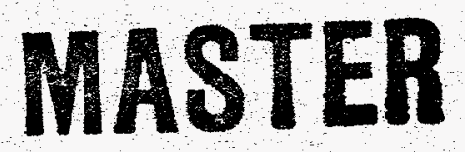

DISTRIBUTION OF THIS DOCUMENT IS UNLIMITEO

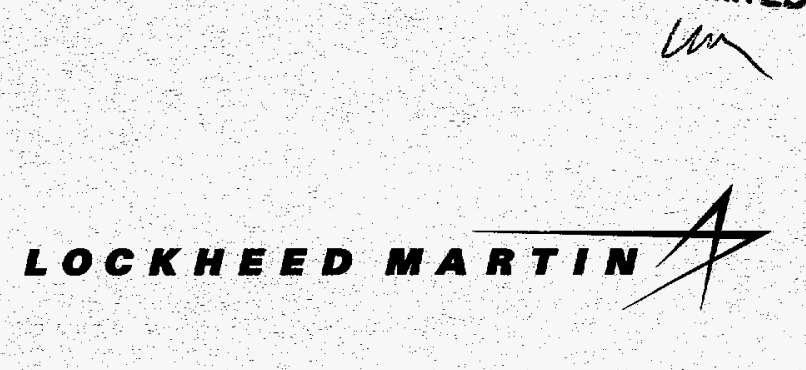




\section{DISCLAMMER}

Portions of this document may be illegible in electronic image products. Images are produced from the best available original document. 


\section{DISCLAIMER}

This report was prepared as an account of work sponsored by an agency of the United States Government. Neither the United States Government nor any agency thereof, nor any of their employees, makes any wartanty, express or implied, or assumes any legal liability or responsibility for the accuracy, completeness, or usefulness of any information, apparatus, product, or process disclosed, or represents that its use would not infringe privately owned rights. Reference herein to any specific commercial product, process, or service by trade name, trademark, manufacturer, or otherwise does not necessarily constitute or imply its endorsement, recommendation, or favoring by the United States Government or any agency thereof. The views and opinions of authors expressed herein do not necessarily state or reflect those of the United States Government or any agency thereof. 


\title{
Uncertainty Analysis of the SWEPP PAN Drum Assay System for Glass Waste (Content Codes 440, 441, and 442)
}

\author{
Larry G. Blackwood \\ Yale D. Harker \\ Teresa R. Meachum \\ Woo Y. Yoon
}

Published October 1996

Idaho National Engineering Laboratory

TRU Waste Programs

Lockheed Martin Idaho Technologies

Idaho Falls, Idaho 83415

Prepared for the

U. S. Department of Energy

Office of Environmental Restoration and Waste Management

Under DOE Idaho Operations Office

Contract DE-AC07-94ID13223 


\begin{abstract}
The Idaho National Engineering Laboratory is being used as a temporary storage facility for transuranic waste generated by the U. S. Nuclear Weapons program at the Rocky Flats Plant (RFP) in Golden, Colorado. Currently, there is a large effort in progress to prepare to ship this waste to the Waste Isolation Pilot Plant (WIPP) in Carlsbad, New Mexico. In order to meet the TRU Waste Characterization Quality Assurance Program Plan nondestructive assay compliance requirements and quality assurance objectives, it is necessary to determine the total uncertainty of the radioassay results produced by the Stored Waste Examination Pilot Plant (SWEPP) Passive Active Neutron (PAN) radioassay system. This paper discusses a modified statistical sampling and verification approach used to determine the total uncertainty of SWEPP PAN measurements for glass waste (content codes 440,441 , and 442) contained in 208 liter drums.

In the modified statistical sampling and verification approach, the total performance of the SWEPP PAN nondestructive assay system for specifically selected waste conditions is simulated using computer models. A set of 100 cases covering the known conditions exhibited in glass waste was compiled using a combined statistical sampling and factorial experimental design approach. Parameter values assigned in each simulation were derived from reviews of approximately 100 real-time radiography video tapes of RFP glass waste drums, results from previous SWEPP PAN measurements on glass waste drums, and shipping data from RFP where the glass waste was generated. The data in the 100 selected cases form the multi-parameter input to the simulation model. The reported plutonium masses from the simulation model are compared with corresponding input masses. From these comparisons, the bias and total uncertainty associated with SWEPP PAN measurements on glass waste drums are estimated. The validity of the simulation approach is verified by comparing simulated output against results from calibration measurements using known plutonium sources and two glass waste calibration drums.
\end{abstract}




\section{SUMMARY}

This report describes a methodology for determining the total uncertainty of the SWEPP PAN drum assay system and applies this methodology to the measurement of glass waste (content codes 440,441 , and 442). In order to perform this evaluation, a complete assessment of the physical and radiological characteristics of content code 440,441 , and 442 waste drums was completed by reviewing real time radiography (RTR) tapes, data from past measurements using the PAN assay system, and shipping records from Rocky Flats Plant. Based on the data analysis, a set of 100 computer simulated glass waste drums were prepared for input into a computer simulation of the assay system response and analysis routines. Parameters which could alter the response of the assay system were identified and modeled in the simulated waste drums. The list of parameters includes mass of weapons grade $\mathrm{Pu}, \mathrm{Pu}$ contained in fines vs. chunks, mass fraction of Pu-240, fill height of the drum, matrix density, elemental composition of the matrix, absorber index, background singles counts, and background coincidence counts. The elemental composition of the simulated drums was modeled to match as closely as possible that found in the actual drums selected for RTR review. The remaining parameters were assigned based on either random sampling from statistical distributions (derived from the data analysis) or a factorial experimental design.

The computer simulation of the PAN assay system response involved a neutron transport calculation using the Monte Carlo Neutron Photon (MCNP) code and a follow-up program which uses the MCNP results along with other parameters in the statistical model to determine the singles and coincidence rate responses. These data were then analyzed to yield $\mathrm{Pu}$ mass using exactly the same algorithms as contained in the PAN analysis program. The accuracy of the combined simulation routine was verified using data taken from measurements involving two glass waste calibration drums and known $\mathrm{Pu}$ sources. From these comparisons it was determined that small amounts of bias were occurring in the simulation results. Correction factors for the bias were determined using linear regression. These correction factors were included in the computer simulation routine in all analyses involving the simulated waste drums so that there was no bias in the simulation results relative to the expected PAN results.

The results from the analysis of the simulated waste drums indicate that for glass waste the PAN system measurements are biased low by $24 \%$ and the standard deviation varies quadratically from about $2 \mathrm{~g}$ at a nominal $1 \mathrm{~g}$ of weapons grade $\mathrm{Pu}$ to $36 \mathrm{~g}$ at $200 \mathrm{~g}$.

Using the results of this evaluation one can make comparisons with performance criteria as established in Table 9-1 of the TRU Waste Characterization Quality Assurance Program Plan (QAPP) (U. S. Department of Energy, 1995). The parameter of interest in this table is the "Total Uncertainty" quality assurance objective. The performance criteria are given in terms of total alpha activity, but we can make a limited comparisons based on Pu mass. The data show that the performance criteria (based on Pu mass only) can be met for PAN measurements on drums containing glass waste for measured mass values in the range $8 \mathrm{~g}$ to $50 \mathrm{~g}$. However, recently proposed changes in the QAPP requirements (in which only the bias component of the total uncertainty has limits imposed) can be met for all measured values.

In order to be critically safe there is a safety limit that the measured Pu mass plus $2 s_{\mathrm{\varepsilon}}$ (i.e., the measured $\mathrm{Pu}$ mass plus 2 times the standard deviation of the measurement) must not exceed $200 \mathrm{~g}$. The results indicate that the measured mass for drums containing glass waste must be below $77 \mathrm{~g}$ in order to meet this criterion. 


\section{ACKNOWLEDGMENTS}

The authors would like to acknowledge the support of others who have made significant contributions to the production of this report. Dr. L. V. East developed the data files and data listings containing the results of previous PAN measurements pertaining to the glass waste and background measurements. P. J. Taylor reviewed the real time radiography video tapes and assessed the physical condition of the glass waste forms. G. K. Tedford and the SWEPP assay staff provided technical support related to the RTR tapes review. G. K. Becker and Dr. L. V. East provided technical insight based on their experience with the PAN and other assay measurements. Dr. M. J. Connolly is the project sponsor and the authors would like to thank him for his support and encouragement during the course of this evaluation. 


\section{CONTENTS}

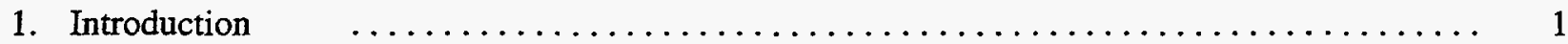

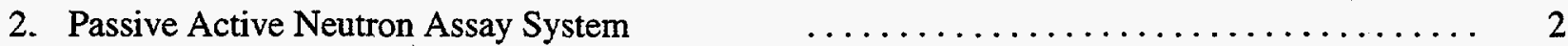

3. Calibration of the PAN Assay System $\quad \ldots \ldots \ldots \ldots \ldots \ldots \ldots \ldots \ldots \ldots \ldots \ldots \ldots$

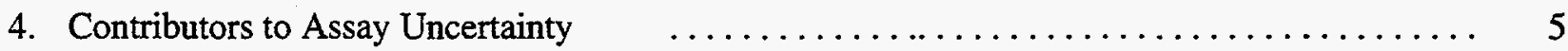

5. Uncertainty Analysis Approach $\quad \ldots \ldots \ldots \ldots \ldots \ldots \ldots \ldots \ldots \ldots \ldots \ldots \ldots \ldots \ldots \ldots \ldots$

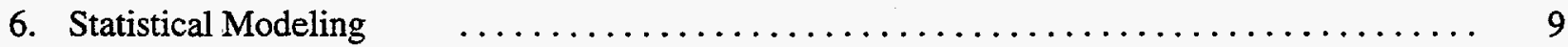

7. Simulation of the PAN Passive Mode Response $\quad \ldots \ldots \ldots \ldots \ldots \ldots \ldots \ldots \ldots \ldots \ldots \ldots$

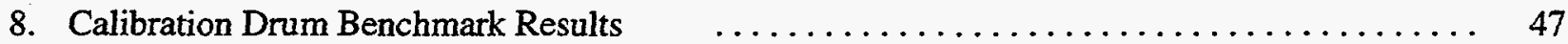

9. Uncertainty Analysis Using the Simulated Waste Drum Sample Results $\ldots \ldots \ldots \ldots \ldots$

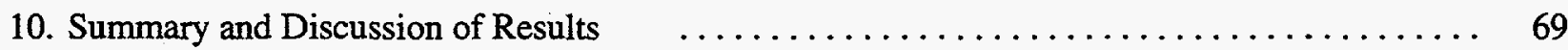

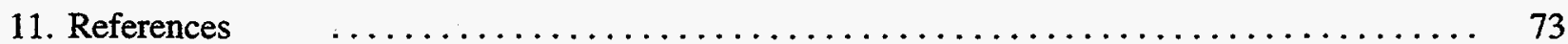

Appendix A-Computer Programs for PAN Assay System Calculations $\quad \ldots \ldots \ldots \ldots \ldots \ldots$

Appendix B-Differences Between the Statistical Modeling for the Glass Waste $\quad \ldots \ldots \ldots \ldots .92$ vs. Combustible Waste Uncertainty Analyses

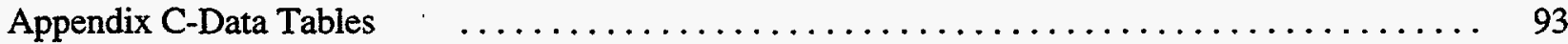




\section{Uncertainty Analysis of the SWEPP PAN Assay System for Glass Waste (Content Codes 440, 441, and 442)}

\section{INTRODUCTION}

The Passive Active Neutron (PAN) radioassay assay system at the Stored Waste Examination Pilot Plant (SWEPP) is used to certify waste drums in terms of quantifying plutonium ( $\mathrm{Pu}$ ) and other transuranic element activities. At this time, assay reports produced by the system provide uncertainty values which are based solely on the counting statistics of the assay technique. It is recognized that, depending on the waste form involved, there can be significant additional systematic and random errors that need to be quantified before the total assay uncertainty can be quoted. This report presents a generalized uncertainty analysis of the PAN assay system measured plutonium mass for 208 liter waste drums containing plutonium contaminated glass waste. Glass wastes comprise content codes 440 (glass vials), 441 (unleached raschig rings), and 442 (leached raschig rings).

Ideally this uncertainty evaluation would be carried out experimentally by systematically varying each parameter to determine its effect on the measured plutonium mass. However, because there are a large number of parameters which can affect the assay results and some of these parameters are correlated, it is not practical to carry out a full experimental uncertainty evaluation. Therefore, a calculation/simulation approach was devised to achieve the same goal. While the general process applies equally to active interrogation and passive coincidence counting, it has only been applied to passive coincidence counting at this time.

The structure of this report will focus on stepping through the uncertainty analysis process for glass waste. As background information, a brief review of the PAN assay system is presented in Section 2 . This is followed in Section 3 by a review of the original calibration of the PAN assay system performed by Los Alamos National Laboratory (LANL). Section 4 identifies the major contributors to assay uncertainties. In Section 5 the basic approach used to evaluate the PAN assay system uncertainty is described. Section 6 describes the statistical modeling involved in setting up the simulated glass waste assay cases. Section 7 steps through the process of simulating the PAN passive mode response. To address the nuances associated with coincidence counting, the theory of coincidence counting as applied in the PAN assay system is also presented. The uncertainty analysis results are given in Section 8 where the simulation approach is benchmarked against measured data taken with two glass waste calibration drums and Section 9 which presents the findings from the analysis of 100 simulated waste drums. Section 10 summarizes the results and presents the final uncertainty assessment with regard to plutonium assay for drums containing glass waste. 


\section{PASSIVE ACTIVE NEUTRON ASSAY SYSTEM}

The SWEPP PAN system is a second generation passive active neutron system developed in the early 1980 's by Los Alamos National Laboratory for the U. S. Department of Energy (DOE) and delivered to the Idaho National Engineering Laboratory (INEL) in 1983. This system was designed to assay drums containing transuranic contaminated waste. Later a similar system was built by LANL and delivered to the INEL for the purpose of assaying boxes containing transuranic waste. Even though much of the formalism will apply to both the drum and box assay systems, the specifics in this report will address only the drum system. The SWEPP PAN assay system is described in an INEL internal document (Becker, 1993a); for more details the reader is referred to that document. A summary description is given below.

The PAN assay system consists of a shielding housing which surrounds the drum on all four sides, top and bottom. Each side of the housing contains moderator (i.e., graphite, polyethylene), thermal and lowenergy neutron shielding (i.e., cadmium, boron) and He-3 neutron detectors. There are two types of detector assemblies contained in each side of the assay system; i.e., bare detectors and shielded detectors.

The shielded detectors are grouped into detector packages where each package is surrounded by thermal and low-energy neutron shield consisting of cadmium and borated rubber. Inside the cadmium and borated rubber are three or four He-3 neutron detectors surrounded by polyethylene. This type of detector assembly is sensitive to fast neutrons and insensitive to thermal and low-energy neutrons. The bare detectors are also He-3 detectors surrounded by polyethylene but are not shielded by cadmium or borated rubber. In this configuration they are sensitive to all neutrons.

The assay system operates in two modes, passive and active. In the passive mode the detector assemblies (bare and shielded) are detecting neutrons produced by spontaneous fission and $(\alpha, n)$ interactions in the waste matrix. Differentiation between the fission neutrons and the $(\alpha, n)$ neutrons is accomplished by coincidence event counting. In this type of counting, a coincidence event is recorded when two neutrons are detected by the system within a specified time window. There are two coincidence windows used: one is $35 \mu$ s long and looks for coincidence events from the shielded detectors in the enclosure and the other is $250 \mu \mathrm{s}$ long and looks for coincidence events from all detectors (shielded and bare) in the enclosure.

In addition to the coincidence counting, single event counting is also accumulated during the passive mode. The single event counting data are used to derive chance coincidence corrections to the coincidence data and also to arrive at a Moderator Index (MI).

In the active mode the shielded detectors are used to detect neutrons produced by stimulated fission resulting from thermal neutron interrogation. The interrogation neutron source for the active mode is a Zetatron $14 \mathrm{MeV}$ neutron generator located at one corner inside the system shield enclosure. The high energy neutrons are moderated to thermal via the graphite moderator in the enclosure walls and varying amounts of moderator in the waste matrix. For the active mode the signal of interest is taken from a gated count of the shielded detectors for the time window from $700 \mu \mathrm{sec}$ to $2700 \mu \mathrm{sec}$ following each neutron burst from the neutron generator. This time window was selected to allow the fast neutrons from the generator to thermalize in the enclosure and thereby have a higher probability to stimulate fission in the Pu-239 and at the same time the thermalized interrogation neutrons are not detectable by the shielded detectors. To account for background, another count window is opened from $5.7 \mathrm{msec}$ to $15.7 \mathrm{msec}$ after 
each neutron burst. It is expected that during this time window only background neutrons will be present.

Also during the active mode two monitors are used to monitor the interrogation neutron flux and the effective transmission of interrogation neutrons through the contents of the drum. The first monitor, called the cavity monitor, consists of one bare $\mathrm{He}-3$ detector mounted inside the cavity along an upper corner. The second monitor, called the barrel flux monitor, is a single $\mathrm{He}-3$ detector mounted at the center of the back wall of the assay system enclosure inside a cadmium collimator so that the detector's field of view is the center of the drum. These two monitors are gated with the same time window as the shielded detectors during the active mode. The ratio of the cavity monitor count during active mode to the barrel monitor count during active mode is referred to as the Absorber Index (AI).

The Moderator Index (from the passive mode count) and the Absorber Index (from the active mode count) are used in the analysis algorithm to arrive at correction factors which are intended to correct for moderator and absorber effects on the measured responses (both active and passive responses). The corrected responses are used to determine the measured plutonium mass. Therefore, both the active and passive counts must be completed to obtain the needed correction factors. Three measured mass values are obtained by the system for each measurement sequence (passive count + active count); i.e., a mass value determined from the active mode count, a mass value determined from the passive short-gate coincidence count and a mass value determined from passive long-gate coincidence count. However, not all three values are valid over the mass range and waste forms covered in SWEPP waste. A set of selection algorithms is included in the system software to determine which of the three assay values should be used in the waste certification documentation. For most waste, only the two passive mass values are considered for selection as the reported mass. The selection criterion for the reported passive mass is based on which of the short-gate and long-gate coincidence mass values has the smaller relative error. 


\section{CALIBRATION OF THE PAN ASSAY SYSTEM}

The basic calibration of the PAN system is performed using standard sealed neutron sources in an empty waste drum. The original calibration was performed by LANL prior to delivery of the PAN system to the INEL. It has been checked repeatedly since as part of the SWEPP operational quality control program.

Listed below are the basic equations used by the PAN assay system to determine the mass assay values.

Mass $_{A}=C_{A}(\text { Net Shielded Count })_{A} C F_{A}$

Mass $_{\mathrm{lg}}=\mathrm{C}_{\mathrm{lg}}(\text { Long-Gate Coincidence Rate })_{\mathrm{P}} \mathrm{CF}_{\mathrm{lg}}$

Mass $_{\mathrm{sg}}=\mathrm{C}_{\mathrm{sg}}(\text { Short-Gate Coincidence Rate })_{\mathrm{P}} \mathrm{CF}_{\mathrm{sg}}$

where:

Mass $_{\mathrm{A}}=\mathrm{Pu}$ mass as determined from the active mode,

Mass $_{\mathrm{lg}}=\mathrm{Pu}$ mass as determined from the passive mode and the long-gate coincidence method, Mass $_{\mathrm{sg}}=\mathrm{Pu}$ mass as determined from the passive mode and the short-gate coincidence method, $\mathrm{C}_{\mathrm{A}}, \mathrm{C}_{\mathrm{lg}}, \mathrm{C}_{\mathrm{sg}}$ are the base calibration coefficients for the active, long-gate coincidence, and shortgate coincidence modes, respectively, and

$\mathrm{CF}_{\mathrm{A}}, \mathrm{CF}_{\mathrm{lg}}, \mathrm{CF}_{\mathrm{sg}}$ are the matrix correction factors for the active, long-gate coincidence, and shortgate coincidence modes, respectively.

The effects of waste matrix, etc. on the system response were estimated during the original calibration series and an algorithm for determining the correction factors was developed by LANL. The correction factors were determined empirically using simulated waste drums in which generic materials (e.g., vermiculite, boric acid, sand, and metal scraps) were used to simulate the waste matrix. The basic assumption in the development of the simulated waste was that the matrix was uniform, the source distribution was uniform and that each waste drum was filled to near the volume capacity of the drum. Over the years, there have been small changes made to the correction factor algorithm, but the basic premises; i.e., uniform matrix and uniform source distributions, have not changed. 


\section{CONTRIBUTORS TO ASSAY UNCERTAINTY}

The PAN system itself reports a counting statistics error based on the detector response values and an assumed Poisson distribution for counts. Beyond the counting statistics errors, there are various other contributors to the total uncertainty of a measurement. Properly designed, the total uncertainty analysis will include the effects of all these factors in addition to the counting statistics.

\subsection{Base Calibration}

In the base calibration, the system response is measured for a well characterized neutron source (i.e., known neutron strength and elemental and chemical composition) at specified positions in an empty waste drum. There are three primary uncertainties associated with the base calibration. The first is the uncertainty in the source strength which includes any decay corrections which are applied and the number of neutrons produced per decay. The second is the uncertainty about the elemental and chemical composition of the neutron source material. The elemental and chemical composition can significantly affect the reported neutron source strength by producing an unknown number of neutrons from $(\alpha, n)$ interactions in the source. The third uncertainty is due to the counting statistics associated with the base calibration data acquisition.

\subsection{Matrix and Source Effects}

The PAN algorithm assumes a uniform matrix and uniform source distribution in the material being assayed. To the degree to which this assumption is violated in a particular waste form, matrix and source configuration effects can be a major contributor to total uncertainty. Listed below are some specific ways that real waste may differ from the uniform matrix and uniform source premise.

1. Source isotopic/chemical composition variability.

2. Non-uniform matrix absorption.

3. Non-uniform matrix moderation.

4. Non-uniform source distribution.

5. Variations in source particle size.

6. Significant voids in the matrix.

7. Shadow shielding of one region by high neutron absorption in another region.

8. Waste elemental composition not addressed by the calibration routine.

\section{$4.3(\alpha, n)$ Source Interference}

In addition to the errors due to nonuniformity in the matrix and source configurations, there are also uncompensated effects resulting from $(\alpha, n)$ reactions occurring in the waste. Since the $(\alpha, n)$ reactions only produce one neutron per reaction, the coincidence counting method in the passive mode should differentiate between neutrons produced by fission (more than one neutron per fission) and neutrons produced by $(\alpha, n)$ reactions. However, the coincidence counting method will have a contribution due to accidental or chance coincidences. There are standard techniques to correct the coincidence counting data for these spurious events and these techniques work well when the corrections for chance coincidence is small compared to the real coincidence rate. In those cases where the $(\alpha, n)$ source strength is clearly dominant over the fissile neutron source strength (i.e., the chance coincidence rate is dominant over the true coincidence rate) there is a very large uncertainty associated with the correction for chance coincidence events. 
In addition, high count rates will also lead to counting losses which are not compensated for in the simple correction applied in the assay system analysis routine. For example, the standard corrections applied for counting losses are based on the assumption of random events and are not applicable to correlated events as is the case in coincidence counting. The random event based corrections are valid when the correction is small but not when the counting loss is the same order of magnitude as the basic count rate. There are drums at the Radioactive Waste Management Complex (RWMC) where the neutron count rate is high enough that this circumstance applies. Under these situations the corrections are considered suspect and contribute significantly to the overall uncertainty of the measurement.

Thus $(\alpha, n)$ interference and counting losses can be sources of significant uncertainties in the assay results. In fact, recent experience has indicated that these effects can be the dominant contribution to the uncertainty of the passive assay results. 


\section{UNCERTAINTY ANALYSIS APPROACH}

The large number of parameters with potential significant effects on assay quality precludes evaluating the PAN system's uncertainty by actual physical experimentation. Instead a calculation/simulation method was used. This computer intensive approach involves three main components: an input data set consisting of statistically generated waste drum and related measurement parameters, a computer model for the physical processes associated with each PAN assay measurement (neutron transport and temporal response), and a set of computer programs duplicating the PAN system's data processing routines (from which counting data and final mass values are obtained).

The statistical modeling process is described in Section 6 of this report. The neutron transport and temporal response calculations are performed using a benchmarked neutron transport model based on the Monte Carlo Neutron Photon (MCNP) transport code (Briesmeiser, 1986). The computer programs used to process the final data are described in Appendix A.

The analysis approach described below follows the same general approach as that previously developed and-reported for combustible waste (Harker et al., 1996). Some modifications to the previous methods were made either to accommodate differences in the matrix configurations encountered with glass waste or to enhance the realism of the calculations involved in the simulation process. Descriptions of these changes can be found in either Appendix A (for computer programs) or Appendix B (for statistical modeling).

\subsection{Basic Steps in Performing the Uncertainty Analysis}

The basic steps in performing the uncertainty analysis are as follows:

1. Perform a content code specific review of probable causes of error in the PAN measurements to identify those factors which need to be included in the uncertainty analysis.

2. Review real-time radiography (RTR) video tapes, a data base of previous PAN assay results, and information provided by the waste shipper to establish either statistical distributions or appropriate values for factorial design settings for the parameters considered to have an effect on the assay results.

3. Develop a composite statistical model incorporating the variances and correlations of the distribution-based parameters identified in (1) and (2).

4. From the statistical model developed in (3), generate sets of simulated values for the distributionbased parameters by random sampling. Combine these values with assigned values for the factorial design variables to produce a complete set of parameter settings for each of 100 simulated waste drums.

5. For each simulated waste drum in the set from (4), apply the MCNP model of the PAN assay system to determine the basic efficiencies and temporal responses of the assay system.

6. For each case in the set from (4), input the results from the MCNP model produced in (5) and other parameters from the statistical model into the simulation routine of the assay system response to determine the basic counting data that would be produced by the PAN assay system under the conditions specified.

7. Apply the PAN assay system analysis algorithm to the set of counting data produced in (6) to determine the measured plutonium mass. Compare that mass to the mass used as input to the simulation process (i.e., the true plutonium mass).

8. Analyze the results from all the specified cases to arrive at the total uncertainty which can be assigned to the measured plutonium mass for the waste form being studied. 
As a check on the validity of the simulation approach for a particular content code, the simulation process is benchmarked against actual measurements on calibration drums. Each calibration drum is an actual drum built of known contents to approximate the matrix configuration found in a typical drum of the waste form under study. Measurements of the calibration drum using the PAN system are obtained with $\mathrm{Pu}$ sources with known properties placed in the drum. The quantities and locations of the Pu sources are varied from run to run. The calibration runs are also computer modeled using the same basic approach as that for the simulated waste drums. Comparison of the simulated PAN results to the known PAN results for the calibration runs thus provides a check on the validity of the simulation process. 


\section{STATISTICAL MODELING}

By modeling the relevant parameters affecting measurement system response, we are effectively creating simulated waste drums and associated conditions (e.g., background measurements) for input into the MCNP code and subsequent calculations. This chapter describes how the values for the various parameters for each simulated waste drum were obtained. Parameters are characterized into two types (primary and secondary) within which values may be assigned in either of two basic ways (modeled based on a random sample from a probability distribution, or determined by a factorial experimental design). These distinctions are discussed further in the next two sections.

Table 6-1 shows a list of the parameters determined to be important in a review of the PAN measurement process for glass waste. The parameters are broken down according to type and assignment method.

Table 6-1. Breakdown of parameters by type and assignment method.

\begin{tabular}{|l|l|l|}
\hline \multirow{2}{*}{ Assignment method } & \multicolumn{2}{|c|}{ Parameter type } \\
\cline { 2 - 3 } & \multicolumn{1}{|c|}{ Primary } & \multicolumn{1}{|c|}{ Secondary } \\
\hline \multirow{2}{*}{ Modeled } & Matrix density & Pu spatial distribution \\
& Elemental matrix composition & Shielded $(\alpha, n)$ singles rate $\left(\mathrm{A}_{\mathrm{n}}\right)_{\mathrm{sh}}$ \\
& Fill height & System $(\alpha, \mathrm{n})$ singles rate $\left(\mathrm{A}_{\mathrm{n}}\right)_{\mathrm{sy}}$ \\
& Pu-240 mass fraction & Waste drum background measures: \\
& Absorber index & Short gate coincidence rate $\left(\mathrm{B}_{\mathrm{c}}\right)_{\mathrm{sg}}$ \\
& Daily background drum measures: & Long gate coincidence rate $\left(\mathrm{B}_{\mathrm{c}}\right)_{\mathrm{lg}}$ \\
& Short gate coincidence rate $\left(\mathrm{B}_{\mathrm{c}}\right)_{\mathrm{sg}}$ & Shielded singles rate $\left(\mathrm{B}_{\mathrm{s}}\right)_{\mathrm{sh}}$ \\
& Long gate coincidence rate $\left(\mathrm{B}_{\mathrm{c}}\right)_{\mathrm{lg}}$ & System singles rate $\left(\mathrm{B}_{\mathrm{s}}\right)_{\mathrm{sy}}$ \\
& Shielded singles rate $\left(\mathrm{B}_{\mathrm{s}}\right)_{\mathrm{sh}}$ & Daily background drum measures: \\
& System singles rate $\left(\mathrm{B}_{\mathrm{s}}\right)_{\mathrm{sy}}$ & Short gate coincidence rate error \\
& & $\left(\sigma_{\mathrm{bc}}\right)_{\mathrm{sg}}$ \\
& & Long gate coincidence rate error \\
& & $\left(\sigma_{\mathrm{bc}}\right)_{\mathrm{g}}$ \\
\hline Design & Pu mass in chunks vs. fines \\
\hline
\end{tabular}

\subsection{Parameter Types}

For the purpose of generating the simulated waste drums, parameters can be considered to be of two basic types, primary and secondary. Primary parameters are characteristics such as fill height, density, plutonium quantity, and certain background measurements whose determinations do not depend on other characteristics (although they may be correlated with one another). Secondary parameters are those which are in some way dependent on the nature of the primary parameters. This includes such characteristics as the distribution of Pu chunks within the drum which cannot be specified until fill height, the number of chunks, etc. are determined, and shielded $(\alpha, n)$ singles rate which is closely related to total $\mathrm{Pu}$ quantity. The dependence of the secondary parameters on the primary parameter values requires that the statistical analysis and sampling be accomplished in two stages. First empirical data relative to the primary parameters are analyzed and appropriate values for the simulated drums determined. Once these values are obtained, they are incorporated as input into the analysis of the secondary parameter information so that the secondary simulated values can be calculated. (Note, that the secondary parameters generally have a random component in addition to their dependence on the primary parameters.) 


\subsection{Methods of Assigning Parameter Values}

Parameter values for the simulated waste drums were assigned either based on statistical modeling followed by random sampling or in a configuration specified by a factorial experimental design. To distinguish between parameters whose values are assigned using the modeling approach vs. the experimental design approach, we use the terms modeled parameters and design parameters respectively. In most cases we prefer to use the modeling approach whenever possible to keep the calculated uncertainty to the most realistic minimum value possible. By modeling both the individual parameter distributions and the correlation between parameters, a rather precise estimate of how the measurement system can be expected to perform over the range of drum configurations expected to be encountered is obtained. It also insures that the matrix configurations derived are realistic.

The experimental design approach is used when more control is desired over the parameter values chosen, or when the underlying distribution can not be modeled (e.g., when appropriated data for detailed modeling purposes are not available). This approach works best when the direction of the effect of the parameter is known (e.g., increasing moisture leads to increasing negative bias), the effects are monotonic, and upper and lower bounds on the likely values for the parameters can be specified. By assigning each of the upper and lower bound values to a portion (usually $1 / 2$ ) of the simulated drums, the parameter's effect on the resulting total uncertainty estimate is maximized. Hence the calculated uncertainty will be conservative (in that true uncertainty will in fact be less than the calculated quantities).

Random samples for most of the modeled parameters values were obtained from probability distributions determined by analysis of available data for the population of interest. In the case of the more complex parameters related to density and elemental makeup of the matrix, the empirical data from a random sample of the existing waste drums were modeled directly, as described below. Pu quantity was treated as a design parameter (although it could have been modeled) for reasons described below.

\subsection{Sample Size}

Selecting the appropriate number of simulated waste drums for a study such as this is not an exact calculation because of both the varied ways in which the data are generated and the varied uses to which the data will be put. Sampling just to obtain a good estimate of the overall bias and variability of the measurement system does not require as many samples as would sampling for sensitivity analysis or other types of analysis that in some way break down the data into smaller components. Other than the uses to which the data are intended, the primary determinant for sample size is the number of parameters being considered. The larger the number of parameters, the greater the sample size needs to be to get the same degree of representation of the population being sampled. For Latin hypercube samples (used for some of the primary parameters as described below) Iman and Helton (1985) suggest a minimum sample size of $n=4 / 3 q$, where $q$ is the number of parameters, but that a value of $n$ between $2 q$ and $5 q$ may be beneficial if the model is not too expensive to run. For the glass waste, it is hard to determine the appropriate reference number of parameters. Table 6-1 lists 10 primary parameters, although the effective number is larger since elemental composition of the matrix is really composed of different combinations of various elements. On the other hand, only a few parameters were determined by Latin hypercube sampling. 
For the glass waste we somewhat arbitrarily chose to simulate $n=100$ drums. It was felt this number would give a good deal of information on many dimensions and the preparation and calculation time for this many simulated cases were reasonable.

\subsection{Primary Modeled Parameters}

Modeled primary parameters for simulated glass waste drum configurations include: mass fraction of $\mathrm{Pu}$ 240 , fill height of drum, matrix density in the drum, elemental composition of the matrix, absorber index, background singles rates (system and shielded), and background coincidence rates (long gate and short gate).

Due to the complexity of the glass matrix, developing a theoretical multivariate statistical distribution from which random samples for which fill height, matrix density, and elemental composition could be obtained was not feasible. Instead, these characteristics were modeled based on empirical sampling. That is, the simulated drums were modeled to match the matrix configurations of a random sample of 100 actual glass waste drums. The random sample was obtained from the population of glass drums at the INEL for which real-time radiography (RTR) tapes exist.

For the other modeled primary parameters (Pu-240 mass fraction, absorber index, and the daily background drum background measurements) values for the simulated drums were obtained by first assigning probability distributions to each parameter. These distributions were determined by analyzing appropriate sources of available empirical data. The statistical distribution for the random component of the absorber index values was derived from the analysis of various sets of replicate PAN measurements. Data on the distribution of background measurements were obtained from records for the daily background drum measurements in the existing PAN data base. Finally, shipping records from Rocky Flats were reviewed to obtain Pu-240 mass fraction information, which varies from production lot to production lot.

Once the probability distributions were assigned, random sampling from those distributions produces representative values for the simulated drums. Details of the analysis processes involved and specific primary parameter results for the glass waste drums are given below.

\subsubsection{Matrix Composition}

To model intra-drum spatial density and material composition variations, the RTR image for each drum in the random sample of 100 drums and its corresponding simulated drum was divided into 24 zones ( 3 radial x 8 axial). The density was determined within each of these zones by using a zone averaged X-ray transmission estimate (i.e., gray scale assessment). These zonal densities were normalized to yield the same overall drum density as determined by dividing the drum net matrix mass by the overall volume occupied by the waste form. The elemental composition for each zone was arrived by identifying specific items (e.g., glass vials, raschig rings, different types of packing material and absorbant, etc.) in the RTR image. Each item identified in a zone was given an elemental composition based on its nominal chemical form. The item-by-item elemental compositions were then combined to produce a zonal elemental inventory.

Many different types of items were identified in the review of the RTR tapes. A list of the item types found appears in Table 6-2. An assessment of the material composition of these items resulted in the 
specification of 27 different atomic elements (excluding plutonium) that can contribute to the composition of a particular drum. These elements are also listed in Table 6-2.

Table 6-2. Material types and atomic elements found in glass waste drums.

\begin{tabular}{|l|l|}
\hline \multicolumn{1}{|c|}{ Material types } & \multicolumn{1}{|c|}{ Atomic elements } \\
\hline Cardboard & Aluminum \\
Clasps & Boron \\
Fiber pack & Barium \\
Glanges & Carbon \\
Light bulb & Calcium \\
Metal pieces, nuts, bolts & Chlorine \\
Oil, dry & Chromium \\
Paint can, metal pieces & Copper \\
Particulate, floor sweeping & Fluorine \\
Polypropylene, polyethylene & Iron \\
Polystyrene, o-ring & Hydrogen \\
Portland cement & Mercury \\
Raschig rings & Potassium \\
Screen & Magnesium \\
Security rings & Manganese \\
Water & Molybdenum \\
Wire & Nitrogen \\
& Sodium \\
& Nickel \\
& Oxygen \\
& Lead \\
& Sulfur \\
& Silicon \\
& Tin \\
& Tungsten \\
& Zinc \\
& Zirconium \\
\hline
\end{tabular}

\subsubsection{Absorber Index}

Absorber index is normally a measured parameter determined in the active phase of the PAN assay. However, the active phase was not simulated in the glass waste case, so the absorber index is treated as a primary parameter. The simulation of absorber index values was based on the assumption that since it is a characteristic of a drum's matrix, its measured value should be very repeatable. Whatever variability that might be observed on repeat measurements of the same drum taken under the same measurement conditions reflects random measurement error (e.g., counting statistics error). Hence information necessary for simulating absorber index values can be obtained by analyzing repeat measurement data. Analysis of repeat data on various drums (calibration drums, background drums, PDP drums, etc.) has born out this assumption. The repeat measurements appear to tightly group around stable means.

Normal probability plots for repeat absorber index data suggests that the data can be approximated adequately by a normal distribution either on the original scale or with log transformed data. The $\log$ transformation has very little effect on the data distributions because the data typically fall in a very small range (e.g., the largest and smallest values in a set of replicates generally differ by much less than a 
factor of two). Fitting normal distributions to the $\log$ transformed data (referred to as log(ai) data from here on) was chosen for the simulation process for two reasons. First, the log transformation tends to stabilize the variance of absorber index measurements across groups of replicate data. Second, it is the $\log$ (ai) values that are actually used in the PAN system calculations, making modeling the log transformed data more directly applicable.

Calibration drum simulations. As described in Section 8, PAN measurements on two glass calibration drums, one for code 440 (glass vials) and one for code 442 (leached raschig rings), were performed and subsequently simulated to benchmark the modeling process. Certain groups of these measurements can be considered to be replicates. Analysis of these replicate data sets provided the mean and variance estimates necessary to generate simulated absorber index values for modeling the calibration drums.

The calibration measurements were divided into replicate groups according to factors which had appreciable effects on $\log ($ ai) means or standard deviations. The means and standard deviations of the $\log ($ ai) values obtained from the content code 440 and 442 calibration drums were considerably different, so separate replicate groups were formed for each. Within the measurements for each content code, the only factors that varied from one measurement to the next were source configuration and positioning of the drum in the chamber (facing the left, right, back, or front of the chamber, or rotated during the measurement). Previous analysis indicated that calibration drum absorber index values do not differ significantly with source quantity or placement, so those factors can be ignored for the purpose of distribution fitting. However, differences in $\log$ (ai) means by drum position are evident in the glass calibration data. Hence different distribution means were calculated for each position. The standard deviation of the data did not differ by position within a content code, so pooled standard deviation estimates were obtained within the two content codes.

The means and standard deviations used for assigning simulated $\log ($ ai) values for the calibration data are given in Table 6-3. For simulating the measurement of a calibration drum from content code $\mathrm{i}$ measured in position $\mathrm{p}$, the simulated absorber index value was generated by the equation

$a i_{S D M}=\exp \left(\mu_{\mathrm{ip}}+\varepsilon_{\mathrm{i}}\right)$

where $\varepsilon_{\mathrm{i}}$ is a random number from a normal distribution with mean 0 and standard deviation $\sigma_{\mathrm{i}}$, and $\mu_{\mathrm{ip}}$ and $\sigma_{\mathrm{i}}$ are taken from Table 6-3.

Position data were missing for one calibration case (run 6 for code 440 ). For that case the overall mean $\log$ (ai) value, 3.47 , was used as the mean for the distribution.

Waste drum simulations. Replicate measurements typically do not exist for actual waste drums. Hence, a slightly different approach was required to obtain the mean and standard deviation values used to generate $\log$ (ai) values for the simulated waste drums. Since the waste drum simulations are based on modeling the matrix characteristics of specific actual waste drums, the mean value for the distribution of the $\log (\mathrm{ai})$ values for each simulated waste drum is set to the measured $\log$ (ai) value obtained by the PAN system for the actual drum being modeled. This insures that the simulated $\log (a i)$ assigned will in fact be reasonable for the type of matrix configuration being modeled. Standard deviation values are assigned as a function of the mean value:

standard deviation $(\log ($ ai $))=.0392 * \log ($ ai $)$. 
Table 6-3. Means and standard deviations for simulating log(absorber index) values for calibration drums.

\begin{tabular}{|c|c|c|c|c|}
\hline Content code & Position & $\begin{array}{l}\text { Sample size } \\
\text { (n) }\end{array}$ & $\begin{array}{c}\text { Mean of } \log (a i) \\
\text { data } \\
\left(\mu_{\mathrm{ip}}\right)\end{array}$ & $\begin{array}{c}\text { Standard deviation } \\
\text { of } \log (\mathrm{ai}) \text { data } \\
\left(\sigma_{\mathrm{i}}\right)\end{array}$ \\
\hline \multirow[t]{5}{*}{440} & front & 5 & 3.48 & \multirow{5}{*}{$\begin{array}{c}.164 \\
\text { (pooled over all } \\
440 \text { positions) }\end{array}$} \\
\hline & left & 7 & 3.55 & \\
\hline & back & 5 & 3.22 & \\
\hline & right & 6 & 3.53 & \\
\hline & rotate & 21 & 3.49 & \\
\hline \multirow[t]{5}{*}{442} & front & 1 & 1.79 & \multirow{5}{*}{$\begin{array}{c}.0335 \\
\text { (pooled over all } \\
442 \text { positions) }\end{array}$} \\
\hline & left & 1 & 1.93 & \\
\hline & back & 29 & 1.88 & \\
\hline & right & 1 & 1.83 & \\
\hline & rotate & - & $-\infty$ & \\
\hline
\end{tabular}

The appropriate function form and coefficients were determined by a regression analysis. For the regression analysis, consideration of data in addition to the glass calibration data was necessitated by the fact that the $\log$ (ai) values for the actual glass waste drums covers a considerably larger range of values than that for the calibration data. The larger range of values is to be expected because the actual waste drums cover many different matrix configurations while there were only two different glass calibration drum matrices. The additional data included in the regression analysis consisted of all readily available absorber index data from PAN system measurements that were repeats or pseudo repeats. Some true repeats were obtained from repeat measurements (with no sources present) of a background drum and the content code 480 (mixed metals) and 440 (glass vials) calibration drums. Additional sets of true repeat measurements were obtained from two Performance Demonstration Plan drums (one empty and one filled with Ethafoam) with fixed matrix and source configurations. Additional pseudo replicates were obtained from the calibration drums runs (with various sources installed) for content codes 300 (graphite), and 330 (combustibles). Each set of replicates is limited to measurements of a specific drum taken in a specific position in the PAN chamber. The number of replicates or pseudo replicates in each of these data sets varied from 2 to 29.

Initial examination of this broader range of data indicated that variability in $\log$ (ai) for replicate measures increased with increasing mean $\log ($ ai) value, but was reasonably constant across content codes and positions (as long as the means were at approximately the same mean level). This lead to the regression analysis to quantify the relationship between the mean $\log (\mathrm{ai})$ and its standard deviation. Because the number of cases going into each replicate data set varied, a weighted least squares analysis was performed (weighting by the square root of the sample size for each replicate set). The original regression model for the data considered intercept, linear, and quadratic terms. The intercept and quadratic terms were not found to be significant in the analysis, hence the final model chosen included only the linear term.

Based on this analysis, the absorber index value for a simulated waste drum is obtained from the model

$a i_{S I M}=\exp \left(\log \left(a i_{P A N}\right)+\varepsilon\right)$ 
where $a i_{\text {PAN }}$ is the actual PAN measured absorber index value for the drum whose matrix is being modeled and $\varepsilon$ is a random number from a normal distribution with mean 0 and standard deviation obtained by substituting $a_{\text {PAN }}$ into Equation 6.2 .

Note that the presence of a significant relationship between mean values and standard deviation seems to contradict the finding that standard deviations did not vary by position for the glass calibration data (since significant mean differences by position were observed). However, in spite of their significance, the mean differences by position were quite small relative to that found over the larger regression data set. Hence over that small range of mean differences, assuming a single standard deviation value for the calibration drum simulations is still appropriate.

\subsubsection{Pu-240 Mass Fraction and Daily Background Drum Background Measurements}

Appropriate probability distributions for $\mathrm{Pu}-240$ mass fraction and the daily background drum background measurements were obtained by the following approach. First, histograms and/or probability plots of the relevant empirical data were reviewed to determine the general shape of the distribution. Guided by this graphical assessment, normal, lognormal, or other standard distributions were fit to the data. Goodness of fit for each distribution was evaluated using the Shapiro-Wilk (SW) statistic (Madansky, 1988) for distributions that transform to normal distributions and chi-square or KolmogorovSmirnov tests for others. For these tests, assessment of goodness of fit is based on the test of the null hypothesis that the data came from the specified distribution. In this case, failure to reject the null hypothesis leads to the conclusion that the data are consistent with the specified distribution. Thus, for example, when testing for a normal distribution, a high $\mathrm{p}$-value suggests the data may be normally distributed, while a low p-value leads to rejection of the hypothesis that the data came from a normal distribution. Generally a p-value of .05 or greater was considered an acceptable fit. The best fitting standard distribution was generally chosen for each parameter. If no satisfactory fit was obtained using the standard distributions, an exponential or other transformation to normality was considered. In one case, that of Pu-240 mass fraction, the empirical distribution itself was used.

To restrict consideration of drum configurations to those combinations of parameters likely to occur in actual glass waste drums, correlations among the daily background drum parameter values were also considered. Correlations indicate the degree of association between two sets of values. There are several ways of calculating correlation. In this case, they were assessed using the Spearman's Rho correlation coefficient (Hogg and Craig, 1978). This correlation value is more appropriate than the usual Pearson's $r$ in cases where the relationships are monotonic but not strictly linear. (Pearson's $r$ is however used in some subsequent comparisons below.) For each correlation, a significance test of the null hypothesis that the true correlation is zero was performed. For a given number of observations, the higher the observed correlation the less likely the true correlation is zero and the lower the probability will be for the significance test. Thus, low p-values, (i.e, $\mathrm{p}<.05$ ) indicate correlations that are statistically significant. Note that it is not necessary to consider the correlations between drum fill height, density, Pu mass, etc. since these parameters are either obtained from direct modeling of actual waste drums (in which case the proper correlations are automatically maintained), or are considered design parameters. Mass fraction is assumed to be uncorrelated with the other measures.

Results from the probability distribution and correlation analyses for mass fraction and daily background drum parameters are summarized in Table 6-4, Table 6-5, and Figure 6-1. Table 6-4 gives the data source, the distribution chosen, and other relevant information for each parameter. Table 6-5 lists pairs of parameters found to have significant $(\mathrm{p}<.05)$ rank order (Spearman's Rho) correlation values. (The results for the background measures apply only to the daily background drum parameters. The waste 
drum background measures are secondary parameters whose generation is described later.)

Figure 6-1 contains histograms of the parameter data along with plots of the fitted distributions. Except for the background singles rate values, which required a non-standard transformation to obtain normality, the histograms are plotted on the original value scale for the parameters. For the background singles rates, the values after the normality transformation was applied are plotted.

For the distributions plotted which are normal distributions or have simple transformation to normality, another indicator of the goodness of fit of the distribution to the data is obtained by considering a normal probability plot for the data (after the appropriate transformation, if any, is applied). Probability plots are inset into the histograms in Figure 6-1.

Table 6-4. Probability distributions for simulated waste drum mass fraction and daily background drum primary parameters.

\begin{tabular}{|c|c|c|c|c|c|c|}
\hline Parameter $^{1}$ & Data source $^{2}$ & $\mathbf{n}$ & $\begin{array}{l}\text { Distribution } \\
\text { selected }\end{array}$ & Parameter 1 & Parameter 2 & $\begin{array}{l}\text { Goodness } \\
\text { of fit }\end{array}$ \\
\hline Pu-240 mass fraction & $\begin{array}{l}\text { Rocky Flats } \\
\text { data }\end{array}$ & 38 & empirical & $\mathrm{NA}$ & NA & NA \\
\hline $\begin{array}{l}\text { Background long gate } \\
\text { coincidence rate }\end{array}$ & $\begin{array}{c}\text { background } \\
\text { drum data } \\
\text { base }\end{array}$ & 106 & normal & mean $=1.01$ & std. dev. $=.134$ & $\begin{array}{c}S W p- \\
\text { value }=.63\end{array}$ \\
\hline $\begin{array}{l}\text { Background short gate } \\
\text { coincidence rate }\end{array}$ & $\begin{array}{l}\text { background } \\
\text { drum data } \\
\text { base }\end{array}$ & 106 & normal & mean $=.0840$ & std. dev. $=.022$ & $\begin{array}{c}\text { SW p- } \\
\text { value }=.18\end{array}$ \\
\hline $\begin{array}{l}\text { Background system } \\
\text { singles rate }\end{array}$ & $\begin{array}{c}\text { background } \\
\text { drum data } \\
\text { base }\end{array}$ & 106 & $\begin{array}{c}y=-3 x^{-3} \\
\text { transforms to } \\
\text { normal }\end{array}$ & $\begin{array}{c}\text { normalized } \\
\text { mean }= \\
-.000127\end{array}$ & $\begin{array}{l}\text { normalized std. } \\
\text { dev. }=.000065\end{array}$ & $\begin{array}{c}\mathrm{SW} \mathrm{p}- \\
\text { value }=.09\end{array}$ \\
\hline $\begin{array}{l}\text { Background shielded } \\
\text { singles rate }\end{array}$ & $\begin{array}{c}\text { background } \\
\text { drum data } \\
\text { base }\end{array}$ & 106 & $\begin{array}{c}y=-3 x^{-3} \\
\text { transforms to } \\
\text { normal }\end{array}$ & $\begin{array}{c}\text { normalized } \\
\text { mean }= \\
-.0194\end{array}$ & $\begin{array}{l}\text { normalized std. } \\
\text { dev. }=.00751\end{array}$ & $\begin{array}{c}\mathrm{SW} \mathrm{p}^{-} \\
\text {value }=.11\end{array}$ \\
\hline
\end{tabular}

1. Background rates refer to the daily background drum measurements only.

2. Rocky Flats data $=$ tabulation of shipping manifests from Rocky Flats. Background drum data base $=$ all existing PAN system data for daily background drum measurements.

Table 6-5. Significant $(p<.05)$ Spearman's Rho correlations in the daily background drum data. ${ }^{3}$

\begin{tabular}{|l|l|c|}
\hline \multicolumn{1}{|c|}{ Parameter 1 } & \multicolumn{1}{|c|}{ Parameter 2 } & Spearman's Rho \\
\hline Background system singles rate & Background shielded singles rate & .88 \\
\hline Background system singles rate & Background long gate coincidence rate & .29 \\
\hline Background shielded singles rate & Background long gate coincidence rate & .38 \\
\hline Background long gate coincidence rate & Background short gate coincidence rate & .37 \\
\hline
\end{tabular}

1. Background rates refer to the daily background drum measurements only.

Sampling plan. Once the probability distributions and correlation structure for the parameters were determined, randomly drawn samples from these distributions produced specific simulated parameter values for the simulated glass waste drums. Various types of random sampling plans can be implemented, including simple random (i.e., Monte Carlo) sampling, stratified random sampling, and Latin hypercube sampling. 
Latin hypercube sampling was used for the $\mathrm{Pu} 240$ mass fraction and daily background drum measurements. Latin hypercube sampling became a part of the general PAN uncertainty analysis approach during the evaluation of the first waste category, graphite. For the graphite case the elemental composition parameters were few and simple enough to be modeled by statistical distributions rather than modeling specific drums as is currently the case. Utilizing Latin hypercube sampling for elemental matrix parameter assignment based on statistical distributions assures the best coverage of the range of waste matrices likely to be encountered. In the current application where, with the exception of Pu 240 mass fraction, waste matrix elemental parameters are assigned by directly modeling matrix characteristics of specific actual waste drums, the use of Latin hypercube sampling is less advantageous. The variability of background measures, absorber index values, etc. about their mean levels are adequately modeled using simple random sampling. For the glass waste analysis, Latin hypercube sampling was maintained primarily for pragmatic reasons. Specifically, it maintains consistency with the previous content code analyses and the software used provided an easy method of imposing the desired correlation structure on the daily background measures.

For this analysis, the Latin hypercube sampling was performed using a computer program developed by Iman and Shortencarier (1984). This FORTRAN 77 program allows the formation of samples from parameters following a number of standard distributions (e.g., normal, lognormal, uniform, loguniform, triangular, and beta) as well as user specified empirical distributions. Furthermore, the program allows specifying a rank order correlation matrix for the input parameters. The correlation structure from this matrix is then imposed on the Latin hypercube sample using the restricted pairing procedure of Iman and Conover (1982).

\subsection{Primary Design Parameters}

\subsubsection{Pu Quantity}

$\mathrm{Pu}$ quantity was the only primary design parameter in the glass waste analysis. Pu quantity data are available in the PAN data base, so could have been modeled. However, the distribution of the Pu quantities in the glass waste drums is concentrated among the smaller quantities. As a result, creating simulated drums with $\mathrm{Pu}$ quantities following the actual distribution would not yield enough information to produce uncertainty estimates for larger $\mathrm{Pu}$ values. To alleviate this problem and to provide better control over how well uncertainty is estimated for crucial quantities such as those given in the Department of Energy's Transuranic Waste Characterization Quality Assurance Program Plan (DOE, 1995), Pu quantity was assigned as part of the experimental design. Specifically, the design specifies 10 levels of Pu quantity, with 10 replicates at each level. The quantities chosen were $1,3,5,10,20,30,40$, 60,80 , and $100 \mathrm{~g}$. Smaller gram quantities were emphasized to provide better uncertainty estimates in the range where most of the actual drum values occur. Quantities less than $1 \mathrm{~g}$ were not considered because that value is approaching the detection limit of the PAN's passive measurement system. The upper limit of $100 \mathrm{~g}$ was chosen to bracket the vast majority of the glass waste drums to be processed at SWEPP. (99.8\% of the measured mass values for glass waste drums in the existing PAN data base were less than $65 \mathrm{~g}$. So even allowing for a large negative bias, the $100 \mathrm{~g}$ upper limit should be more than sufficient to cover the range of true mass values.) 


\subsection{Secondary Modeled Parameters}

Secondary modeled parameters for the simulated glass waste drums consist of the spatial distribution of $\mathrm{Pu}$ fines (by drum zone), the spatial distribution of Pu chunks (height relative to fill height, distance from drum centerline, and angular position in drum), the $(\alpha, n)$ singles rates (system and shielded), the background measures associated with the waste drum measurement (long and short gate background coincidence rates and system and shielded background singles rates), and the daily background drum coincidence rate errors (long gate and short gate). Due to their dependence on the primary parameter results and other factors, various methods other than simple distribution fitting and sampling were often required for the simulation of secondary parameter values. Thus derivation of simulated values for each of the secondary parameters is addressed separately below.

\subsubsection{Spatial Distribution of Pu Chunks}

The expected distribution of Pu chunks in the glass waste is uniform. For drums with $\mathrm{Pu}$ chunks, the spatial location of each chunk was determined by randomly assigning each of three coordinate values: $r$, an angular value between 0 and $360^{\circ}$ (measured from an arbitrary starting point on the circumference of the drum); $c$, a value between 0 and 1 which, when multiplied by $27.87 \mathrm{~cm}$ (the drum radius) for drums without fiber packs or 19.685 for drums with fiber packs (which restrict the diameter of the enclosed waste matrix), indicates the distance from the center axis of the drum; and $\mathrm{z}$, a value between 0 and 1 which is multiplied by the drum fill height to get the vertical distance of the chunk from the bottom of the drum. The values $r$ and $z$ were obtained by sampling from the appropriate random uniform distributions. The value $c$ was obtained by taking the square root of a number generated from a random uniform distribution. (Taking the square root of the number is necessary to obtain uniform spacing over the cylindrical drum volume.)

\subsubsection{Spatial Distribution of Pu Fines}

For drums with $\mathrm{Pu}$ in fines, the distribution within the drum was determined by a random process assuming a lognormal distribution. In the assignment process, it was assumed that the distribution of Pu fines is independent of the total mass distribution in the drum.

To insure a variable but smooth distribution of the fines across the drum, Pu fine quantities were assigned to small subregions of the drum. The fine quantities were then summed up over these small subregions to yield the total fines in each of the 24 modeled drum zones. The subregion size was chosen such that a full drum would have 1,000 subregions. This produced a subregion volume of approximately $207 \mathrm{~cm}^{3}$. The total number of subregions in a simulated drum varies depending on matrix fill height, drum voids, etc. Similarly the number of subregions in each of the 24 modeled drum zones varies according to the zone size and the proportion of the zone filled with waste.

Within a drum, Pu quantity for each subregion was assigned at random from a lognormal distribution with a mean equal to the specified drum total $\mathrm{Pu}$ quantity divided by the number of subregions in the drum, and a variance consistent with drum to drum variability in the PAN database for glass content codes. (That is, the variance of the sum of the subregions for a drum is the same as the between drum variance calculated from the PAN glass database.) Once the subregion values were assigned and summed to obtain the total fines for each of the 24 modeled drum zones, the drum zone values were normalized to insure that the total Pu quantity matched that specified for the drum. 


\subsubsection{System and Shielded $(\alpha, n)$ Singles Rates}

System and shielded $(\alpha, n)$ singles rates have strong dependencies on Pu mass and on each other. For these parameters, simulated waste drum values were obtained by using empirical data for glass drums in the PAN data base to derive regression models for the relationships and then applying those models to the new simulated drum configuration data to obtain appropriate values.

The singles counts are modeled as a function of passive mass. All analysis is done on logarithmic transformations of both the mass and singles count data due to high skewness and because the variability in the data increases dramatically as mass increases. The relationship to mass was estimated from the data base of existing glass waste drums. Only the data for those drums with at least $.5 \mathrm{~g} \mathrm{Pu}$ recorded by the PAN system were used in the analysis. Of the 682 drums in the glass data base, 591 drums met this criteria. Prior to the actual modeling, the data for the content code 440 and 442 were compared to determine if they were similar enough to be combined for the analysis. (Code 441 was not included in the comparison because there were only four such drums in the database.) Regression of the $\log (\alpha, n)$ singles rates on $\log$ (passive mass) generally showed similar results for both waste codes. Tests of significance for differences in slopes and intercepts between the two waste codes did not show significant differences. Also, the correlations of the $\log (\alpha, n)$ singles rates with $\log$ (passive mass) were similar for the two content codes (although slightly stronger for the code 442 drums). As a result it was decided the data could be combined for the analysis.

System and shielded $(\alpha, n)$ counts are highly correlated. To maintain this correlation in the simulated data, the following approach was used. First, a model for the relationship between the $\log (\operatorname{shielded}(\alpha, n)$ singles rate) and $\log$ (passive mass) was developed based on the data base of 591 existing glass waste drums. Using the same data base, a model was also developed for the relationship between the shielded and system rates. Simulated shielded $(\alpha, n)$ singles rates were obtained by randomly sampling from the first distribution. Given these simulated shielded $(\alpha, n)$ singles rates, the corresponding system rates were obtained by random sampling from the second model. This produced simulated rates that are representative of both the marginal distributions of the true $(\alpha, n)$ singles rates as well as their joint distribution (i.e., correlation).

Modeling shielded $(\alpha, n)$ singles rate as a function of mass. Figure 6-2 shows a plot of the $\log ($ shielded $(\alpha, n)$ singles rate) as a function of $\log$ (passive mass).

A general equation for these data that can be used to generate simulated values is of the form:

$\log ($ shielded $(\alpha, n)$ singles rate $)=\alpha+\beta(\log ($ passive mass $))+\varepsilon$

where the parameters $\alpha$ and $\beta$ are the usual regression line parameters and $\varepsilon$ is a random term that produces the variability about the regression line.

The basic regression line equation for these data is:

$\log ($ shielded $(\alpha, \mathrm{n})$ singles rate $)=3.19+.882(\log ($ passive mass $))$

A model for the variability, i.e. the random term $\varepsilon$, is obtained by considering the distribution of the residuals from Equation 6.5. (Residuals are calculated for each point in the data set by subtracting the 
actual value from the predicted values.) These residual values are plotted as a function of $\log ($ passive mass) in Figure 6-3.

Except for a small but potentially important group of negative outliers $(n=14$ cases with values less than -3), the variance of the residuals appears to be fairly constant. (The value of -3 was chosen to define the negative outliers because 3 is approximately the maximum positive residual.) Also, except for the outliers the residuals appeared reasonably normally distributed in a normal probability plot. The standard deviation of the non-outlier residuals is .68. Thus, non-outlier residual values can be simulated using a normal distribution with mean zero and standard deviation .68. A more realistic simulation is obtained by also allowing for the possibility of negative outliers. Hence a compound generation process was employed. Residuals were generated from a normal distribution with mean zero and standard deviation .68 with probability $575 / 589=.976$ (the proportion of non-outliers in the PAN data). With probability $14 / 589=.024$ residuals were randomly selected with replacement from the empirical distribution of the 14 identified outliers. In this manner, outliers should occur in the simulated cases with approximately the same probability and distribution as observed in the actual PAN data.

The complete weighted least squares model for generating $\log ($ shielded $(\alpha, \mathbf{n})$ singles rate) values is then:

$\log ($ shielded $(\alpha, n)$ singles rate $)=3.19+.882(\log ($ passive mass $))+\varepsilon$

where $\varepsilon$ is a randomly generated value obtained as just described. Shielded $(\alpha, n)$ singles rates on the original scale are obtained by exponentiating the $\log ($ shielded $(\alpha, n)$ singles rate) values.

Modeling system $(\alpha, n)$ singles rate as a function of shielded $(\alpha, n)$ singles rate. Figure $6-4$ plots the relationship between shielded and system $(\alpha, n)$ singles rates. Because of the skewness in the distribution of both variables, a log-log transformation, as described above, was considered for the data. However, in this case the data on the original scale exhibited greater linearity and variance homogeneity than the transformed data, so all modeling was done on the original scale.

Following the same approach as the model for predicting shielded $(\alpha, n)$ singles rates from mass, a general equation for these data that can be used to generate simulated values is of the form:

system $(\alpha, n)$ singles rate $=\alpha+\beta($ shielded $(\alpha, n)$ singles rate $)+\varepsilon$

The regression line equation for these data is:

systems $(\alpha, n)$ singles rate $=2.54+4.52($ shielded $(\alpha, n)$ singles rate $)$

The residuals from this regression model proved to vary considerably with shielded $(\alpha, n)$ singles rate. Hence a weighted least squares analysis was performed. The residuals from the final weighted least squares model are plotted in Figure 6-5. A model for the standard deviation of the residuals as a function of shielded $(\alpha, n)$ singles rate was derived by fitting a regression line to the absolute values of the residuals. This yields:

$\sigma_{(\text {stielded })}=.142($ shielded $(\alpha, n)$ singles rate $)$.

(The intercept in the regression model was negative, but not statistically significant. Since standard deviations cannot take on negative values, the intercept was set to zero.) 
The complete model for generating system $(\alpha, n)$ singles rate values is then:

system $(\alpha, n)$ singles rate $=2.54+4.52($ shielded $(\alpha, n)$ singles rate $)+\varepsilon_{\mathrm{N}\left(0_{\sigma \sigma}(\text { (shielded })\right)}$

where $\varepsilon$ is a random value obtained from a normal distribution with mean 0 and standard deviation that depends on the shielded $(\alpha, \mathbf{n})$ singles rate value as given in Equation 6.9.

Bias correction. The relationship between the $(\alpha, n)$ singles rates and mass were modeled based on the measured mass values in the glass data base. However, $(\alpha, n)$ singles values must be assigned to the simulated waste drums based on the true mass values for the drums. Thus, to the extent that the measured mass values are biased, then the assigned $(\alpha, n)$ singles rates will be consistently too high or too low. To account for this potential bias in the assigned $(\alpha, n)$ singles rates, an iterative process was used. Preliminary values were assigned based on the true mass values and the waste drum simulations were evaluated. This indicated a bias in the measurements of approximately $-22 \%$. In the second iteration $(\alpha, n)$ singles rates were then assigned based on $78 \%$ of the true mass value, and the simulations rerun. Since the waste drum simulations are not particularly sensitive to small changes in the $(\alpha, n)$ singles values, applying the bias correction produced little change in the final results.

\subsubsection{Waste Drum Background Measures}

The data in the daily background drum data base were collected over a long period of time, so the variability obtained from sampling from distributions representing these data is characteristic of long term variations in background. To produce variability more consistent with the typical elapsed time between a waste drum measurement and its associated daily background drum measurement (usually a few hours at most), the waste drum background measures are generated based on deviations from the given daily background values.

Obtaining waste drum background counts from daily background values. Background coincidence rates occurring during a waste drum measurement are obtained by sampling at random from a normal distribution with a mean equal to the associated daily background drum value (after applying a correction--see below) and a standard deviation representative of the expected intra-day background variability. The standard deviation was estimated by obtaining six sets of repeated measurement data on specific background and empty calibration drums. Within each of the six data sets, all data were collected on the same day. Hence the data are appropriate for examining intra-day correlations.

The intra-day variance in background counts was calculated for each of the six data sets. The results were then pooled across the data sets to obtain a final variance estimate for the intra-day variability. The square root of this variance is the pooled standard deviation value. Since the data collected for the calculation of these variance values were based on $600 \mathrm{sec}$. measurement times but waste drums are measured for only $200 \mathrm{sec}$., the standard deviations were multiplied by the square root of 3 to get the appropriate standard deviation for use with a simulated waste drum measurement. No multiplier was used in simulating code 440 calibration drum measurements as those measurements were run for $600 \mathrm{sec}$. The code 442 calibration drum measurements were run for $400 \mathrm{sec}$., so the standard deviations were multiplied by the square root of 1.5 for those simulations. The standard deviation values used are listed in Table 6-6. 
Background count rates come from sources outside the detector enclosure (cosmic neutrons, neutrons from within the facility, and natural sources in the soil, etc.) or from cosmic interactions in the materials of the detector enclosure. If the cavity is either empty or has an empty drum in it there is less material in the enclosure to interact (compared to when a waste drum is being measured). The primary interaction for background sources outside the enclosure is neutron scattering and with a matrix inside the drum there is a greater probability to scatter background neutrons and thereby alter their probability of detection by the system detectors. For cosmic sources producing neutrons by interactions in the enclosure walls and enclosure contents, the presence of a matrix will also increase the probability of interaction. Therefore, it can be expected that there will be a difference between a background measurement taken with an empty drum and a background taken with a filled drum. Thus, it is appropriate to apply corrections to the mean when using daily background data from an empty drum to generate values for drums filled with glass materials. These corrections, given in Table 6-6, are multipliers based on the ratio of the means for the code 440 and 442 glass calibration drum measurements to that for the empty drum using the intra-day data just described. Since no code 441 calibration drum was available, the multipliers for code 442 were applied to the code 441 waste drums. (Code 441 wastes are essentially the same as 442 wastes, except that the raschig rings are unleached rather than leached.)

Table 6-6. Mean adjustments and standard deviations for simulating glass waste drum background measures from daily background measures.

\begin{tabular}{|c|c|c|c|c|c|}
\hline \multirow[b]{3}{*}{ Background measure } & \multirow{2}{*}{\multicolumn{2}{|c|}{ Mean adjustment }} & \multicolumn{3}{|c|}{ Standard deviation } \\
\hline & & & \multicolumn{2}{|c|}{ Calibration drums } & \multirow[b]{2}{*}{ Waste drums } \\
\hline & Code 440 & Code 441,442 & Code 440 & Code 442 & \\
\hline Shielded singles rate & .9704 & 1.0013 & .106 & .130 & .184 \\
\hline System singles rate & .9379 & .9654 & .257 & .315 & .445 \\
\hline Short gate coincidence rate & .8868 & 1.0200 & .0131 & .0160 & .0227 \\
\hline Long gate coincidence rate & .9187 & 1.0173 & .0521 & .0638 & .0902 \\
\hline
\end{tabular}

Correlation of intra-day background drum measures. In generating the waste drum background data from the daily background measures, the various background values were assumed to be uncorrelated. This was done in spite of the fact that the background drum data base used to generate the daily background drum measures showed significant correlations between the singles and coincidence rates. However, much of the association is actually due to the difference in background across days and/or drums and is not necessarily applicable to the measures being generated as intra-day deviations from daily background values.

The correlation structure for intra-day background measures was investigated using the same data described above for the pooled variance measures. Rather than test for intra-day correlations separately within each of the six data sets (which would yield very unstable estimates due to the small sample sizes) the data were combined after mean centering each data set. Mean centering removes the effects of the mean differences from the correlations. (Variances were assumed to be equal.) For the centered data, only one significant correlation (either using Pearson's $r$ or Spearman's Rho correlation coefficients) between the various background measures was found, that between the shielded and systems singles rates. Even though significant, that correlation showed an explained variance $\left(R^{2}\right)$ of only $26 \%$. Hence it would seem safe to treat the intra-day background singles and coincidence rates as being independent. Note that a likely explanation for the difference in correlations between daily repeat measurements and longer term time periods is that within a day the variance is mostly due to counting statistics error (i.e., the background really is not changing that much) while for the longer term the background does in fact change. 


\subsubsection{Daily Background Drum Coincidence Rate Errors}

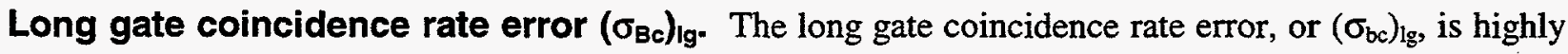
correlated with the long gate coincidence rate, as might be expected. However, it also exhibits significant correlations with the system and shielded background singles rates. Hence, the system and shielded background singles rates where included along with the long gate coincidence rate in a multiple regression analysis to develop a simulation equation for $\left(\sigma_{\mathrm{bc}}\right)_{\mathrm{lg}}$. To account for potential nonlinearities in the relationship, squared terms for each predictor variable were also considered in the analysis.

A backward stepwise regression modeling approach was used to eliminate from the final model any variables without significant relationships to $\left(\sigma_{\mathrm{bc}}\right)_{\mathrm{lg}}$. The modeling process showed that the effects of the shielded $(\alpha, n)$ singles rate were redundant when the other variables were in the model so they were eliminated. The final model selected was

$$
\left(\sigma_{\mathrm{bc}}\right)_{\mathrm{lg}}=.0304+.0387\left(\mathrm{~B}_{\mathrm{c}}\right)_{\mathrm{lg}}+.000352\left(\mathrm{~B}_{\mathrm{s}}\right)_{\mathrm{sy}}-.00416\left(\left(\mathrm{~B}_{\mathrm{c}}\right)_{\mathrm{lg}}\right)^{2}+.00000328\left(\left(\mathrm{~B}_{\mathrm{s}}\right)_{\mathrm{sy}}\right)^{2}
$$

A random term was also included in the prediction, based on the distribution of the residuals from the multiple regression model. A probability plot of the residuals indicated that, except for two outliers, the residual values were approximately normally distributed. It was felt that the outliers were likely due to bad data but were left in the data set to be conservative (i.e., the outliers will produce a larger variance in the generated values).

Thus the final model used for generating the simulated $\left(\sigma_{\mathrm{bc}}\right)_{\mathrm{g}}$ values is Equation 6.11 with an additional random term:

$\left(\sigma_{\mathrm{bc}}\right)_{\mathrm{lg}}=.0304+.0387\left(\mathrm{~B}_{\mathrm{c}}\right)_{\mathrm{lg}}+.000352\left(\mathrm{~B}_{\mathrm{s}}\right)_{\mathrm{sy}}-.00416\left(\left(\mathrm{~B}_{\mathrm{c}}\right)_{\mathrm{gg}}\right)^{2}+.00000328\left(\left(\mathrm{~B}_{\mathrm{s}}\right)_{\mathrm{sy}}\right)^{2}+\varepsilon_{\mathrm{N}(0, .00053)}$

where $\varepsilon_{\mathrm{N}(0, .00053)}$ indicates a randomly generated value from a normal distribution with a mean of zero and a standard deviation of .00053 . The standard deviation value specified was obtained by taking the square root of the mean square error from the multiple regression analysis. (Under an assumption of constant variance, the mean square error is the best estimate of the variance of the residual values.)

Short gate coincidence rate error $\left(\sigma_{\mathrm{Bc}}\right)_{\mathrm{sg}}$. For $\left(\sigma_{\mathrm{bc}}\right)_{\mathrm{sg}}$, no significant correlations other than with $\left(B_{c}\right)_{s g}$ were found. Some improvement in the simulation was obtained by applying a quadratic rather than linear fit to the data. Additionally, a random component was added to simulate the variability about the quadratic fit observed in the data. Figure 6-6 shows the quadratic fit to the data.

A normal probability plot of the residuals for the quadratic fit indicated some lack of fit to a normal distribution, even after eliminating two outliers. The non-normality is due to a slight lack of fit of the quadratic model to the data. In spite of the non-normality, a normal distribution was deemed sufficient for simulation purposes. (Random variability in the error rates isn't a critical issue in the simulations.) Again, leaving in the two outliers in the variance calculation for the normal distribution gives the more conservative results. The simulation model is:

$\left(\sigma_{b c}\right)_{s g}=.00763+.185\left(\mathrm{~B}_{c}\right)_{s g}-.362\left(\left(\mathrm{~B}_{c}\right)_{s g}\right)^{2}+\varepsilon_{N(0, .00012)}$ 
where $\varepsilon_{\mathrm{N}(0, .00012)}$ is a randomly generated value from a normal distribution with mean zero and standard deviation .00012 . (The standard deviation comes from the square root of the mean square error for the quadratic regression.)

\subsection{Secondary Design Parameters}

Pu mass in chunks vs. fines. Initially, RTR tape reviews were used to try and determine the presence of chunks of plutonium in drums and determine their relationship to the total Pu quantity in the drum and their spatial distribution. However, the results of this effort proved to be unsatisfactory (i.e., the presence of chunks, their size and mass, etc., could not be adequately or consistently determined). Hence it was decided to include a parameter in the factorial design part of the simulation simply specifying that Pu quantities in the simulated drums consist of either all fines or all chunks. When all chunks are specified for a drum, each chunk is taken to be $20 \mathrm{~g}$ or the portion thereof required to make up the total quantity of $\mathrm{Pu}$ specified for the drum. For example, drums with $100 \mathrm{~g}$ Pu would have 5 chunks of $20 \mathrm{~g}$ each, while all drums with $20 \mathrm{~g}$ or less total Pu, would have one chunk consisting of the specified quantity (i.e., 1, 3, 5, 10, or $20 \mathrm{~g}$ ). The chunk size configuration was chosen to represent a bounding case. Actual waste drums should fall somewhere between the extreme of all $20 \mathrm{~g}$ chunks and all fines. 
Figure 6-1a. Parameter: Pu-240 mass fraction; Distribution: Empirical

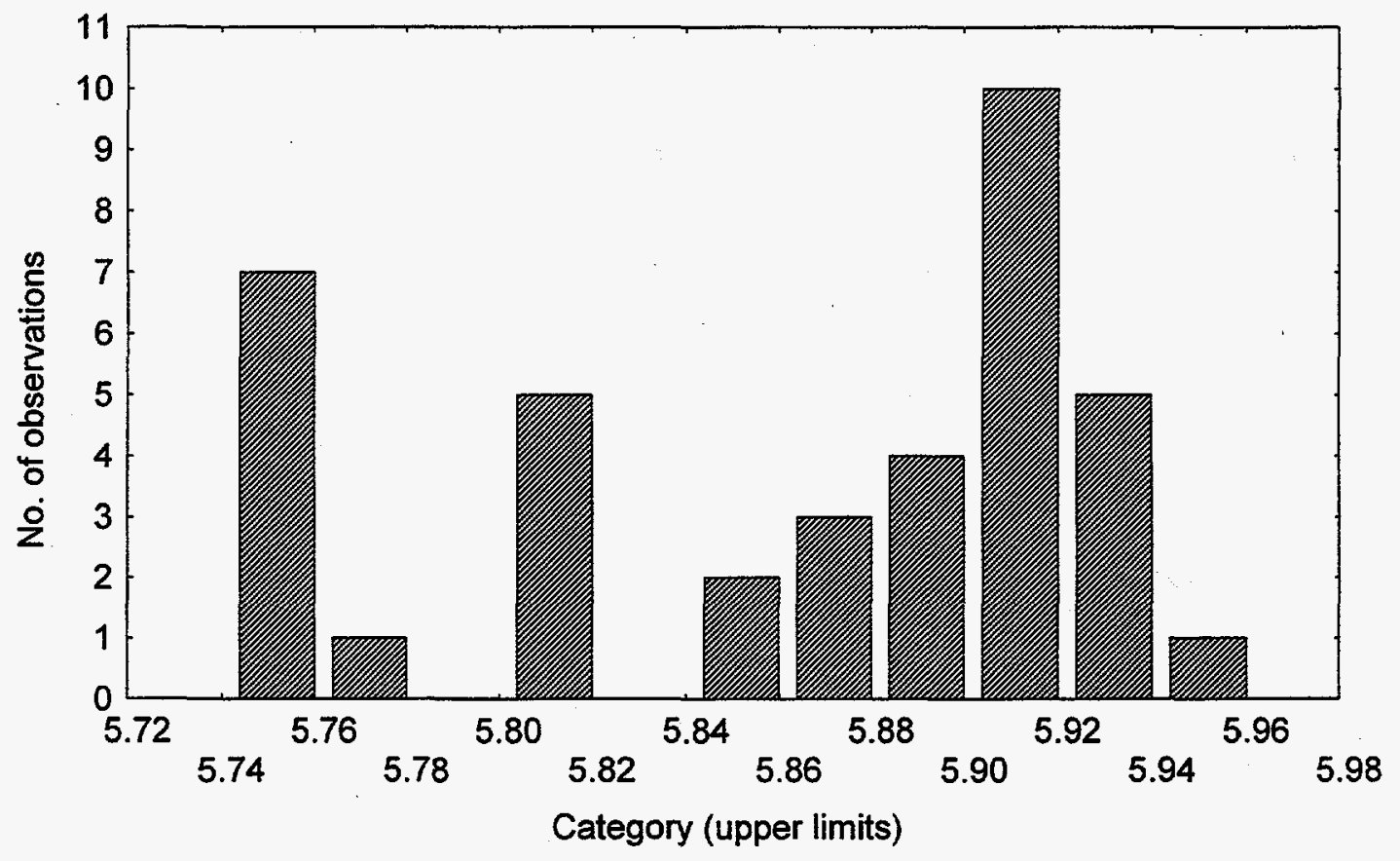


Figure 6-1b. Parameter: Background long-gate coincidence rate Distribution: Normal

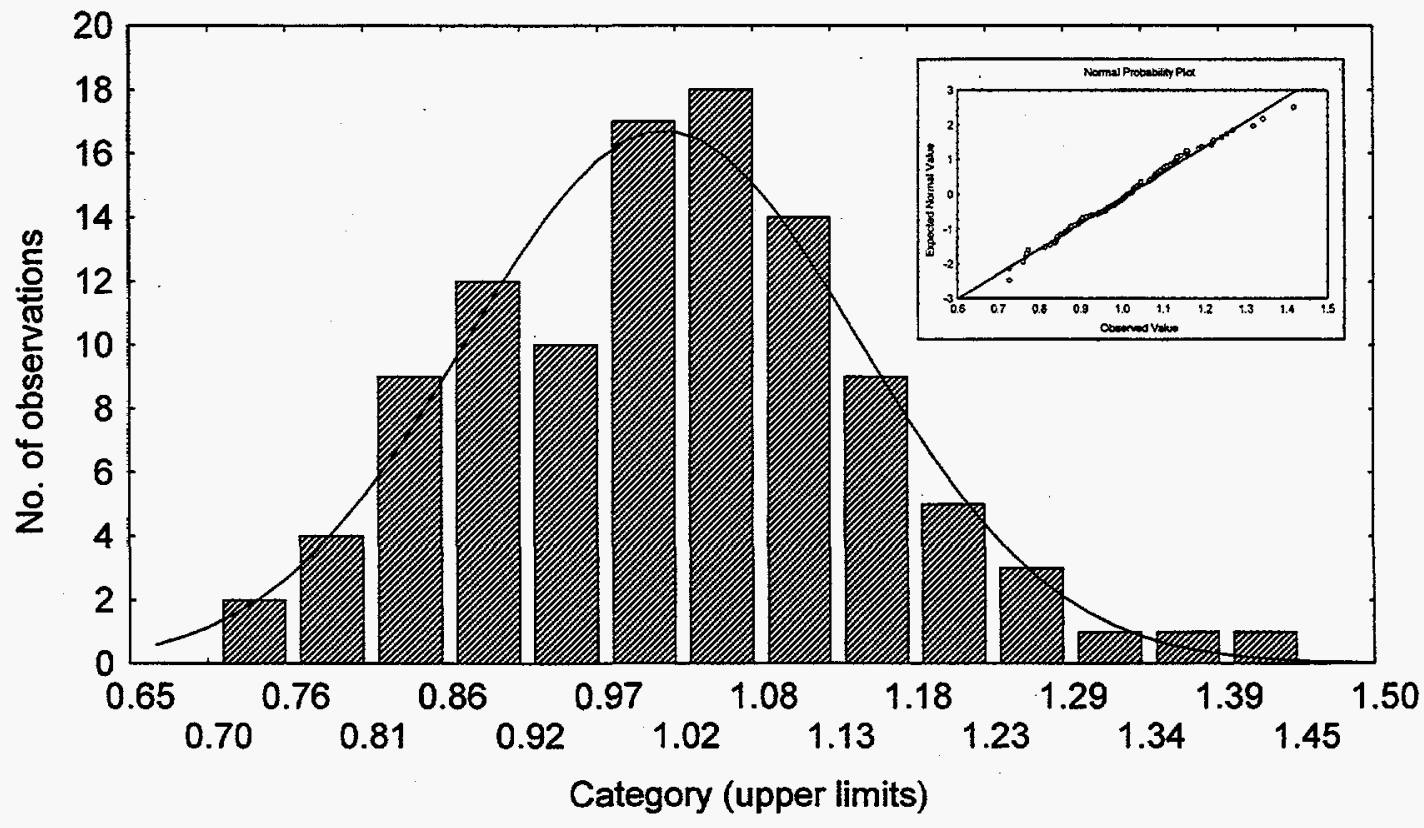

Figure 6-1c. Variable: Background short-gate coincidence rate Distribution: Normal

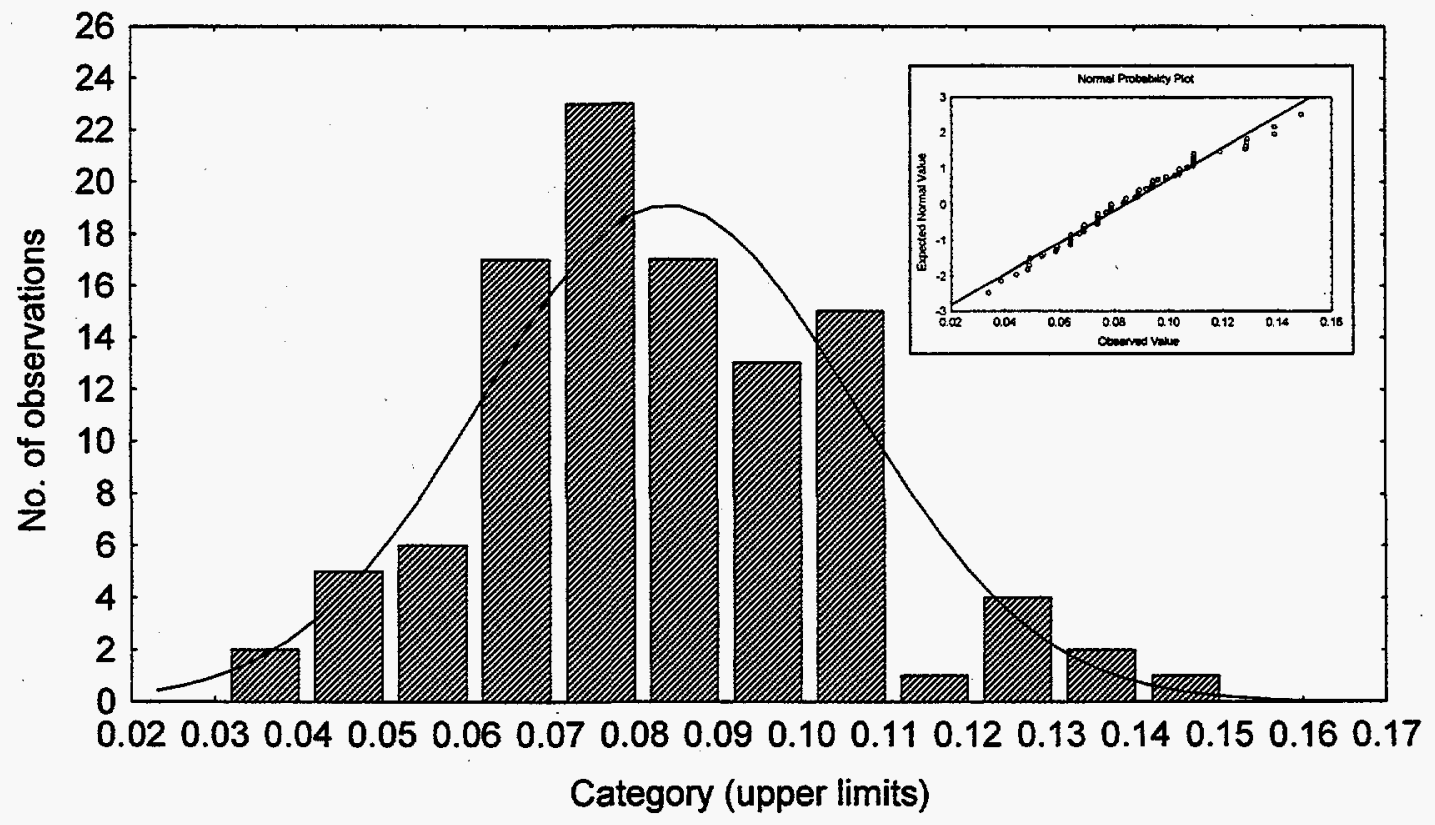


Figure 6-1d. Parameter: Background system singles rate (after transformation)

Distribution: Normal

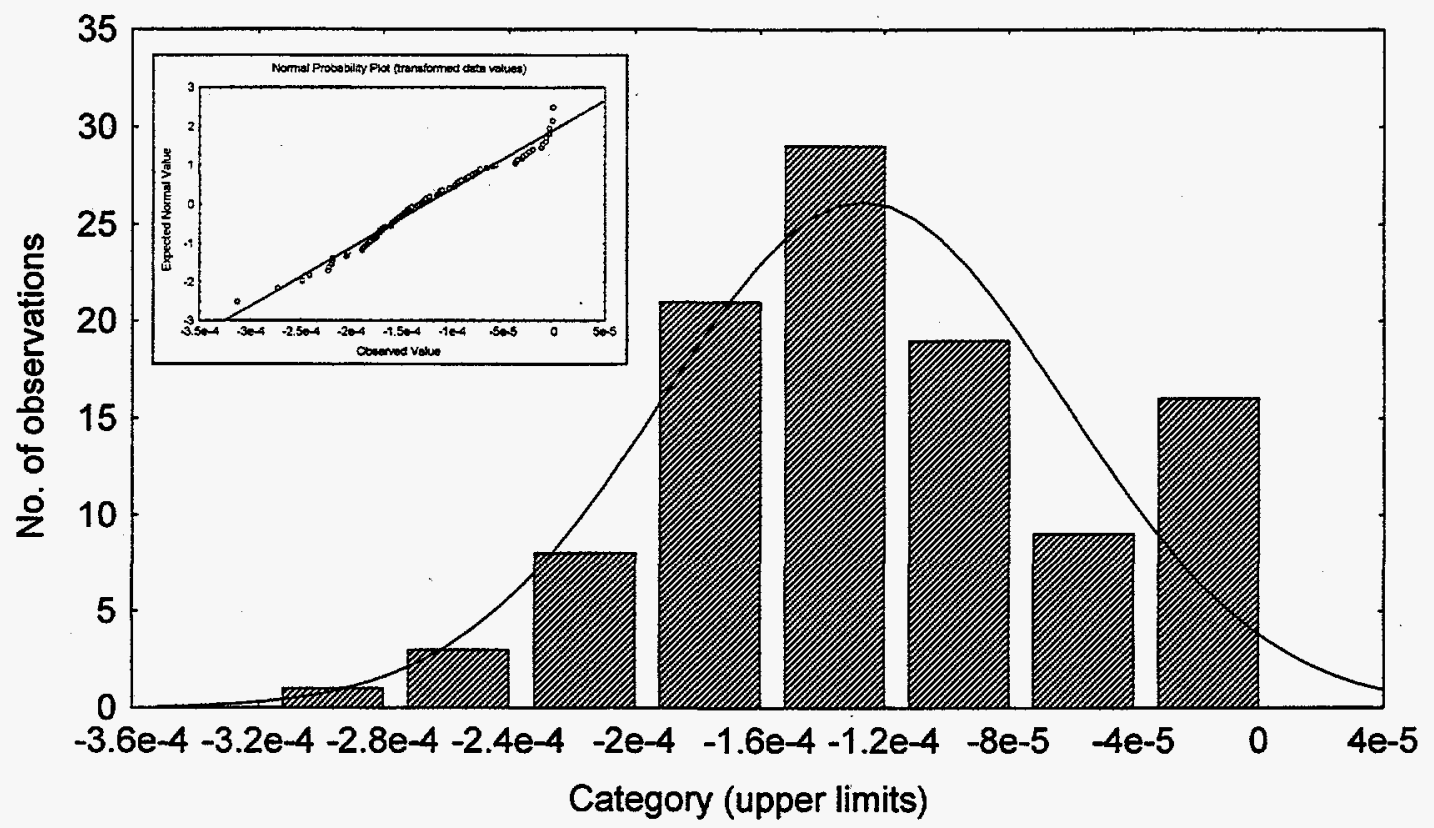

Figure 6-1e. Parameter: Background shielded singles rate (after transformation) Distribution: Normal

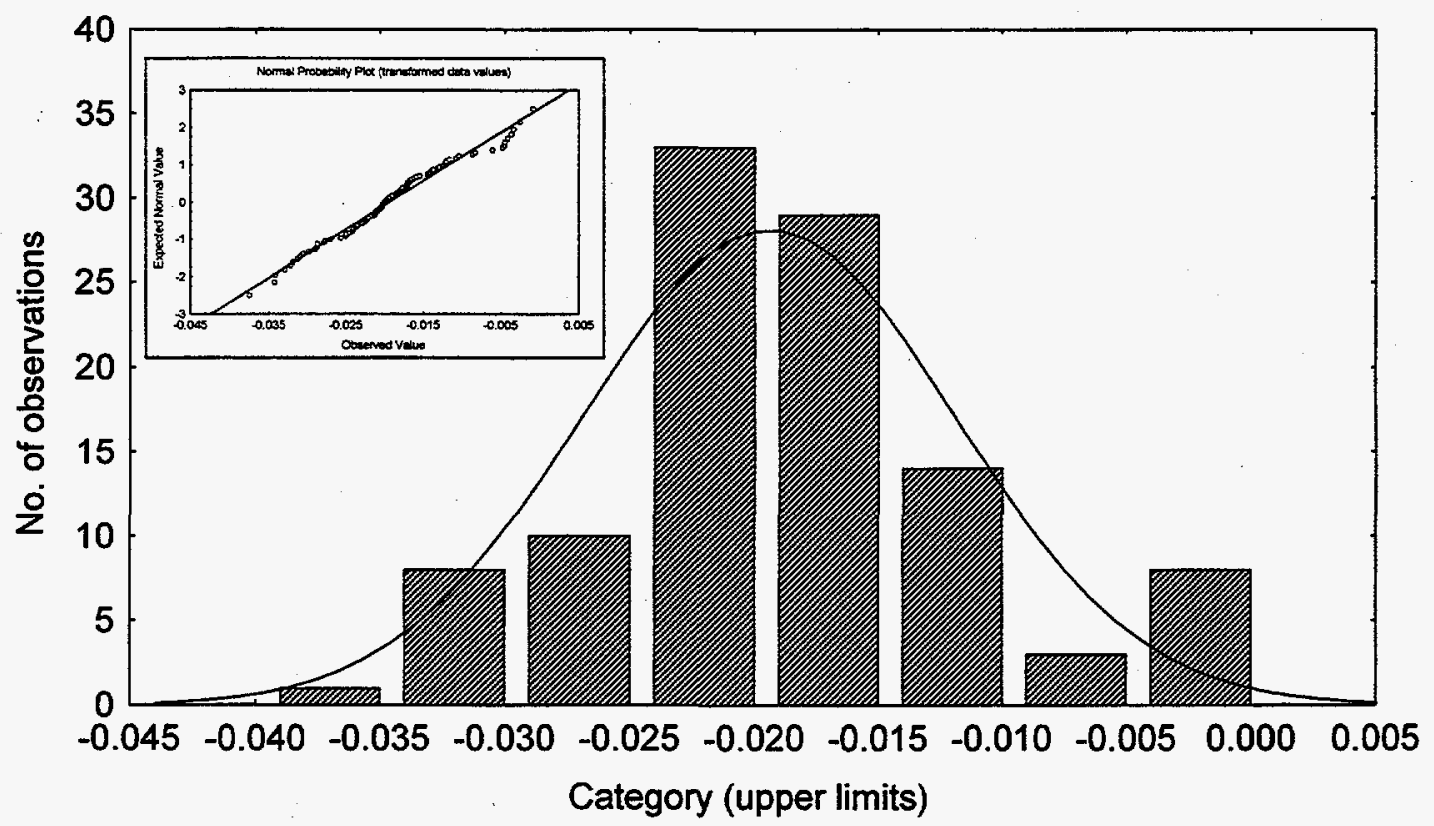




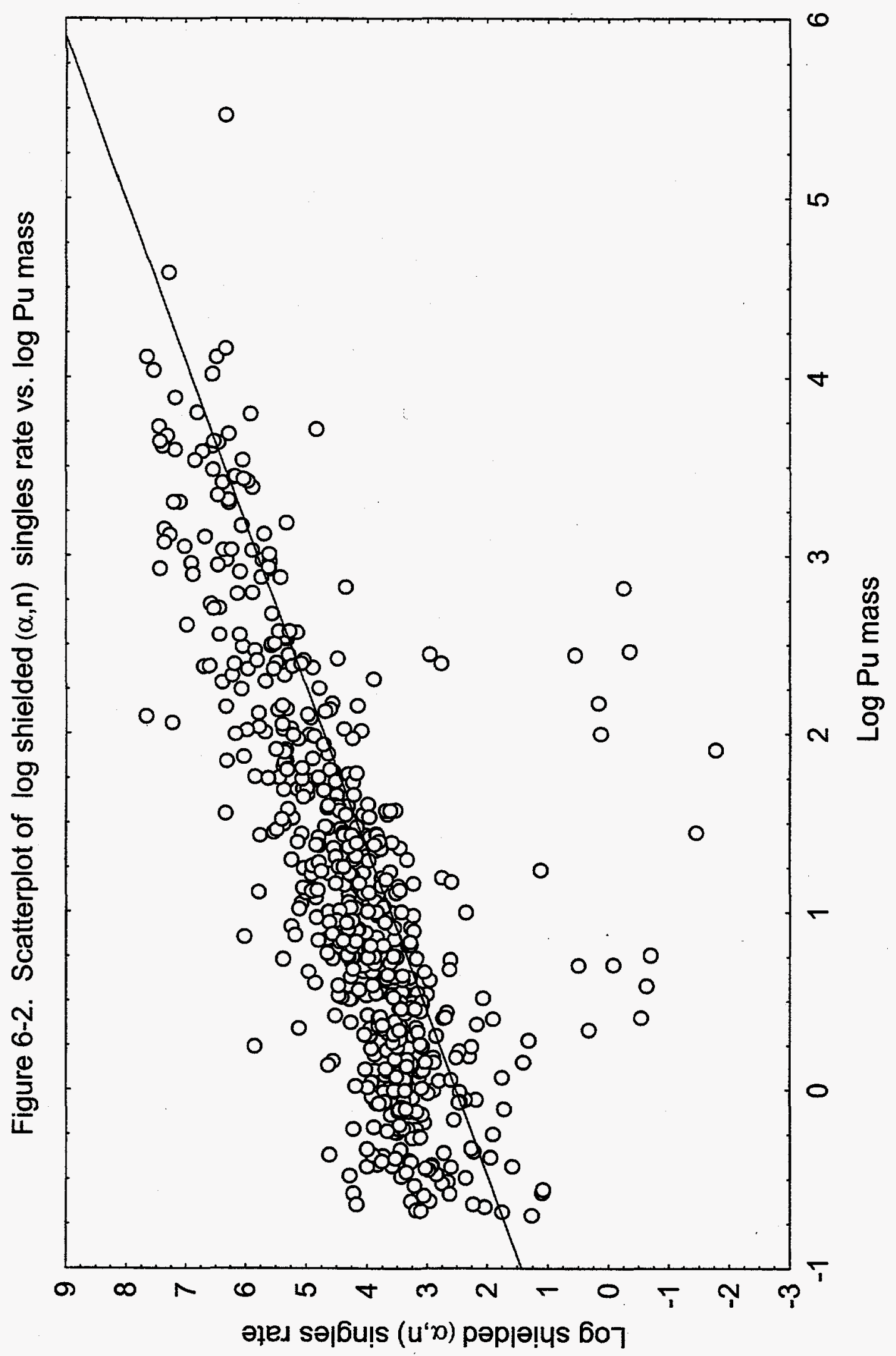


Figure 6-3. Residuals from the regression of $\log$ shielded $(\alpha, n)$ singles rate on log Pu mass

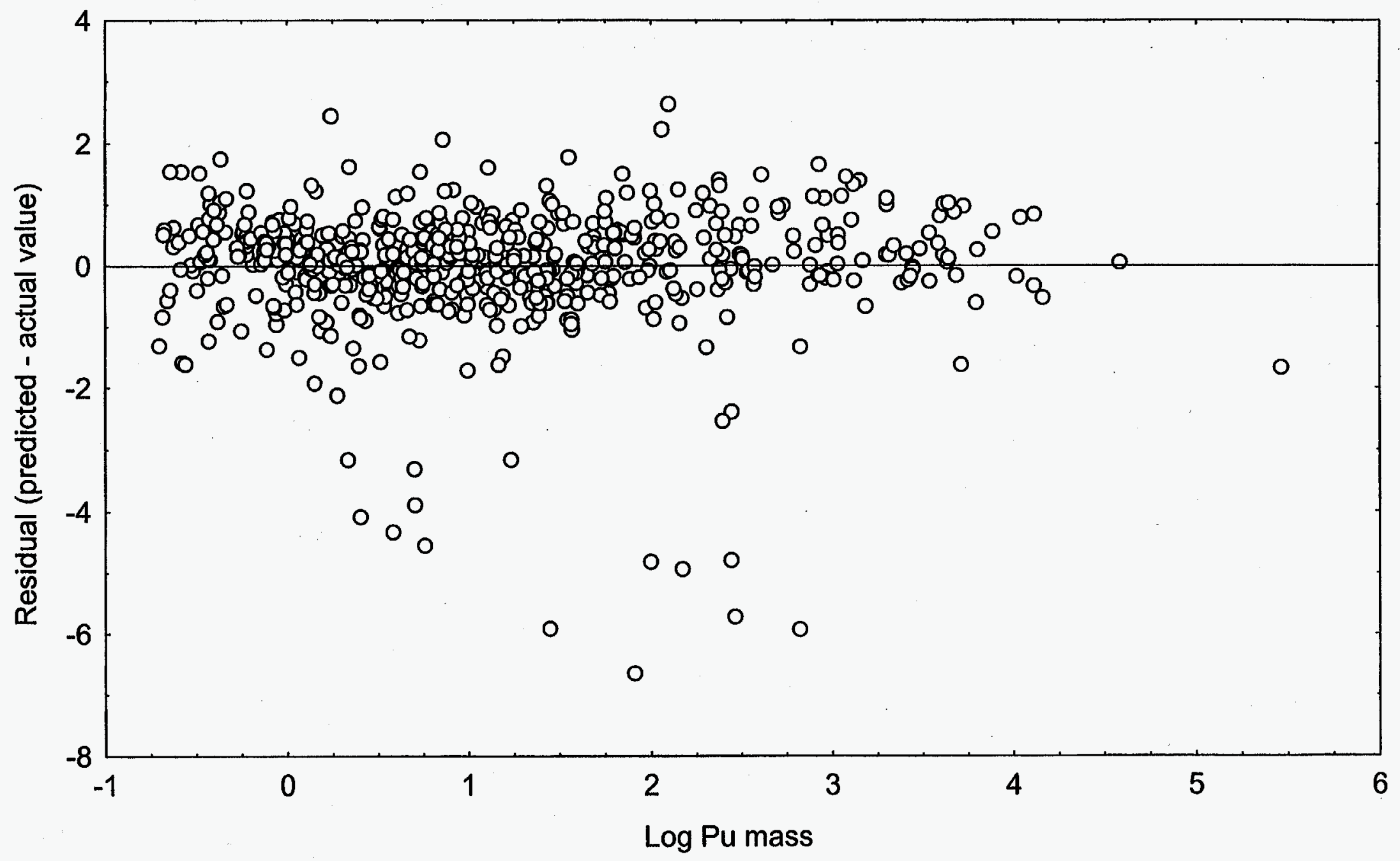


Figure 6-4. Scatterplot of system vs. shielded $(\alpha, n)$ singles rates

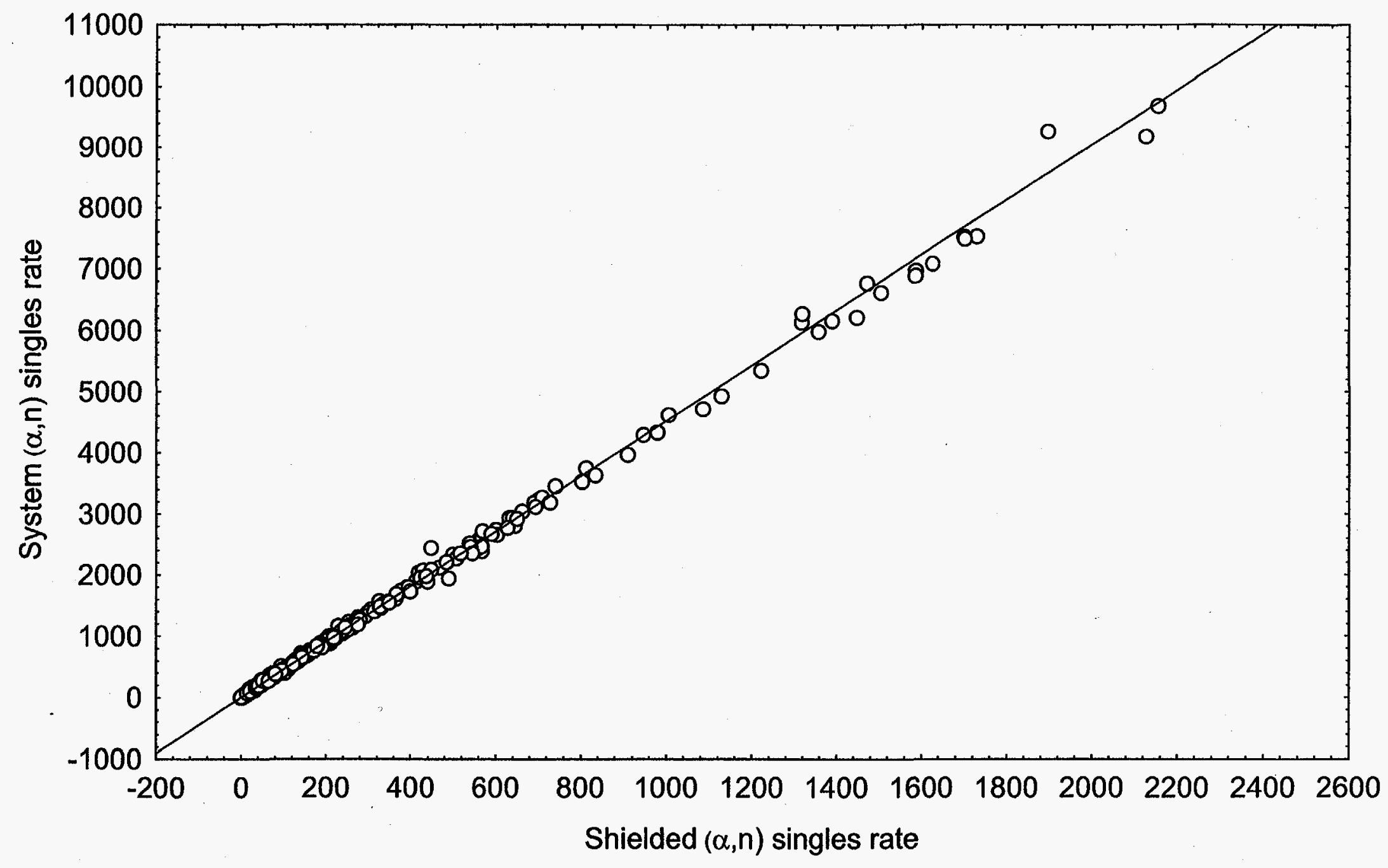




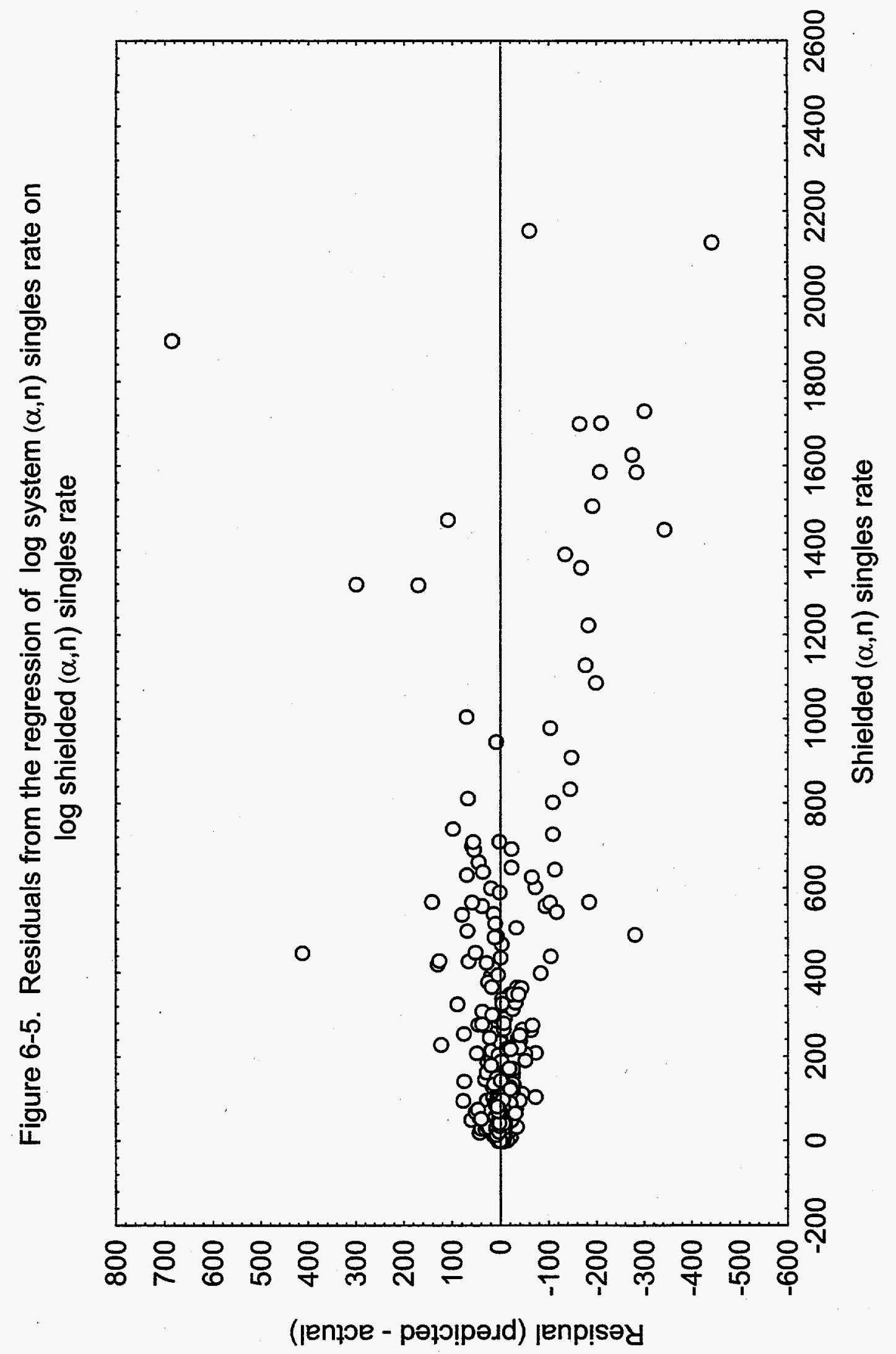




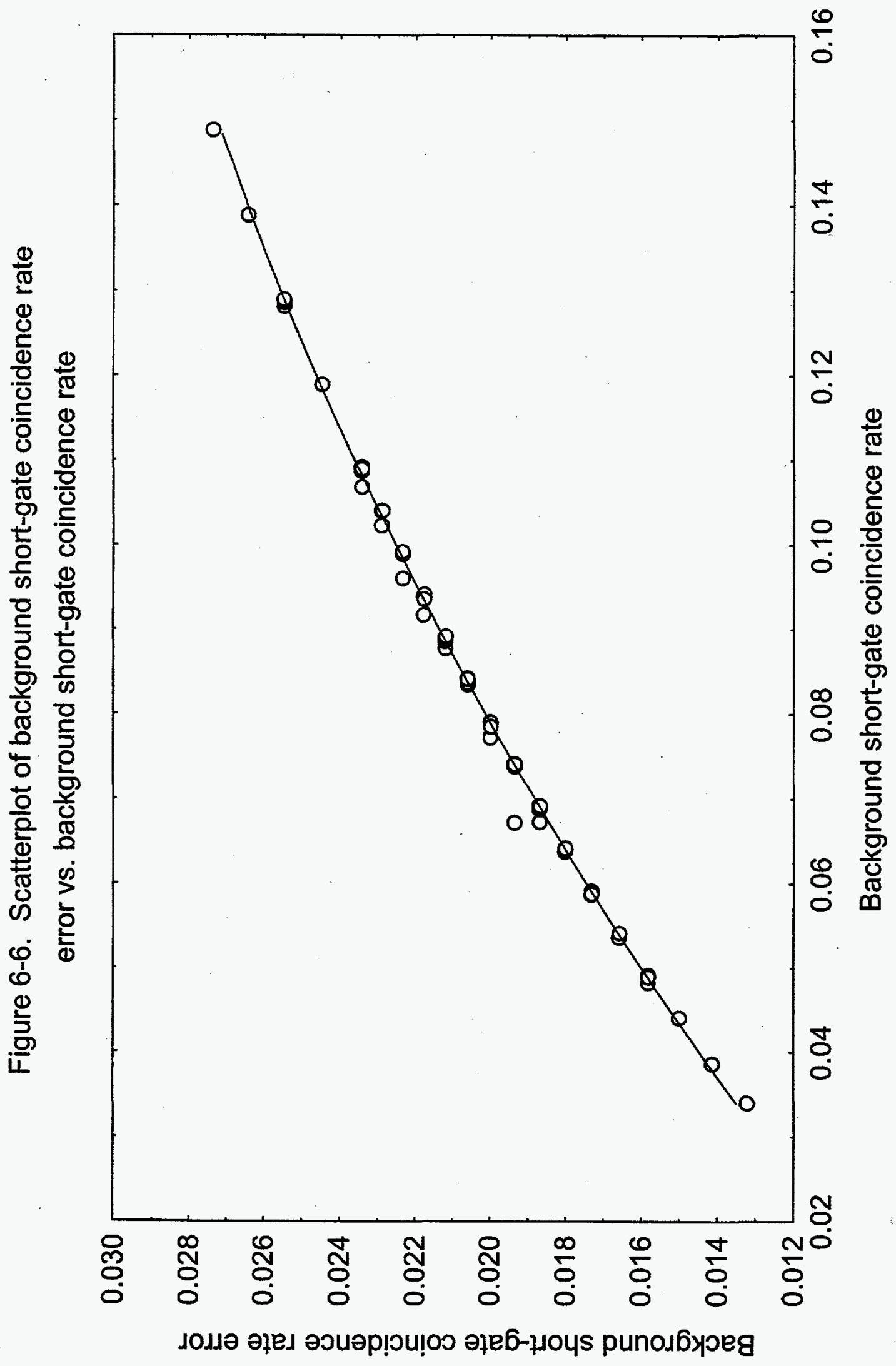




\section{SIMULATION OF THE PAN PASSIVE MODE RESPONSE}

\subsection{Theory of Coincidence Counting}

The PAN assay system passive mode utilizes coincidence counting to differentiate between fission produced events where there are multiple neutrons produced and other nuclear events where only one neutron is produced per event. The type of coincidence system used in the PAN system can best be categorized as a "one-shot coincidence system." In this type of coincidence system a detected pulse opens the coincidence gate and then each pulse detected while the gate is open is counted as one coincidence event. There are two coincidence gate circuits in the PAN system, the first coincidence circuit uses the shielded detectors and is called the short-gate coincidence system. The other coincidence circuit works with all detectors (shielded plus bare detectors) and is called the long-gate coincidence system. For the short-gate coincidence system the gate time is $35 \mu \mathrm{s}$ and for the long-gate coincidence system the gate time is $250 \mu \mathrm{s}$.

\subsubsection{Singles Count Response (Without Counting Loss Included)}

To arrive at the formulae that depict the passive response, it is necessary to start with depicting responses that do not contain counting losses. The basis for the passive response is the spontaneous fission rate of $\mathrm{Pu}-240, \mathrm{~N}_{\mathrm{f}}$.

$\mathrm{N}_{\mathrm{f}}=\lambda_{\mathrm{sf}} \mathrm{N}_{240}$

where

$\lambda_{\mathrm{sf}}$ is the spontaneous fission decay constant for Pu-240,

$\mathrm{N}_{240}$ is the number of atoms of $\mathrm{Pu}-240$ in the drum.

This expression assumes that $\mathrm{Pu}-240$ is the source of the spontaneous fission in the waste drum. There are also small contributions from other even mass plutonium isotopes which can be included in the spontaneous fission effect by adding similar terms or by using $\mathrm{Pu}-240$ effective.

The singles rate (no counting loss) due to fission neutrons, $F$, is determined by:

$\mathrm{F}=\varepsilon \mathrm{N}_{\mathrm{f}} v_{\mathrm{av}}$

where

$\varepsilon$ is the detection efficiency (counts per source neutron), and

$v_{a v}$ is the average number of neutrons produced per spontaneous fission of $\mathrm{Pu}-240$.

The singles rate (no counting loss) due to uncorrelated neutrons is denoted as A. This term contains: (1) counts produced by $(\alpha, n)$ interactions of alpha particles from the decay of plutonium and other transuranics with primarily light nuclei (e.g., oxygen, fluorine, boron) and (2) background counts.

The total singles rate (no counting loss) is denoted as $\mathrm{S}$, where

$\mathrm{S}=\mathrm{F}+\mathrm{A}$. 


\subsubsection{Singles Count Response (With Counting Loss Included)}

The measured singles rate (with counting loss included), denoted as $\mathrm{n}$, is the true count rate, $\mathrm{S}$, multiplied by the fraction of time available for counting, which for a non-paralyzable detector system is:

$$
\mathrm{S}=\frac{\mathrm{n}}{1-\mathrm{n} \delta \mathrm{t}} \quad \text { or } \quad \mathrm{n}=\frac{\mathrm{S}}{1+\mathrm{S} \delta \mathrm{t}}
$$

where $\delta t$ is the dead time of the detector system. (For the PAN system $\delta t$ is $\approx 5 \mu$ s.)

\subsubsection{Coincidence Count Response (Without Counting Loss Included)}

As was the case for the singles response it is instructive to first look at the coincidence response where no counting losses have been included.

The true coincidence rate from fission (with no counting loss), denoted as C, is given in Equation 7.5 for a PAN type coincidence system assuming that the neutron levels decrease with a single die-away constant. Also in the PAN system there is a set delay time between the trigger and gate opening, $t_{d}$.

$$
C=\lambda_{s f} N_{240} \varepsilon^{2} e^{-t_{d} / \tau}\left(1-e^{-T / \tau}\right) \frac{1}{2} \sum_{v=2} G_{v} v(v-1)
$$

where

$v$ is the number of neutrons produced per fission,

$\mathrm{G}_{v}$ is the statistical probability of $v$ neutrons being produced per fission,

$T$ is the coincidence gate time,

$\tau$ is the die-away time of the combined (detector plus drum) system, and

$t_{d}$ is the gate delay time; i.e., the time between the generation of the coincidence gate signal and the opening of the coincidence gate. This delay has been set to be $6 \mu$ s for the PAN assay system.

For Pu-240 spontaneous fission:

$$
\sum_{v=2} \mathrm{G}_{v} v(v-1)=4.102
$$

Equation (7.5) is a familiar expression for a "one-shot" coincidence response for the case of a simple one component die-away system with no counting loss.

\subsubsection{Coincidence Count Response (With Counting Loss Included)}

In order to account for counting losses in the coincidence response it is necessary to start with the number of coincidence gates opened per second and account for how that rate is affected by the dead time of the coincidence system.

The measured coincidence gate rate, $\mathbf{n}_{\mathrm{c}}$, (with counting losses included) is given by: 
$\mathrm{n}_{\mathrm{c}}=\mathrm{n}\left[1-\mathrm{n}_{\mathrm{c}}\left(\mathrm{T}+\mathrm{t}_{\mathrm{d}}\right)\right]$

Substituting Equation (7.4) for $\mathrm{n}$ in Equation (7.7), $\mathrm{n}_{\mathrm{c}}$ can be rewritten as:

$\mathrm{n}_{\mathrm{c}}=\frac{\mathrm{S}\left[1-\mathrm{n}_{\mathrm{c}}\left(\mathrm{T}+\mathrm{t}_{\mathrm{d}}\right)\right]}{1+\mathrm{S} \delta \mathrm{t}}$

Equation (7.8) can be rearranged to determine $n_{\mathrm{c}}$ :

$\mathrm{n}_{\mathrm{c}}=\frac{\mathrm{S}}{1+\mathrm{ST}}$

where:

$$
T^{*}=T+\delta t+t_{d}
$$

There are two types of events counted when the coincidence window is opened. First, there is the "real event" where trigger count which opens the gate and a count during the gate are from the same fission event. Second, there is the "accidental event" where the trigger count which opens the gate and a count during the gate are from different nuclear events occurring at random times. The real coincidence event rate is denoted as " $r$ " and the accidental coincidence event rate is denoted as " $a$ ". The accidental coincidence rate is simply the coincidence gate rate, $n_{c}$ times the average number of counts occurring within a gate window, $T$. This is given by:

$\mathrm{a}=\mathrm{n}_{\mathrm{c}}(\mathbf{n T})=\frac{\mathrm{S}}{1+S T^{*}} \frac{\mathrm{ST}}{1+\mathrm{S} \delta \mathrm{t}}$

If one assumes that $S \delta t<1$, then Equation (7.10) can be approximated by:

$$
\mathrm{a} \approx \frac{\mathrm{S}^{2} \mathrm{~T}(1-\mathrm{S} \delta \mathrm{t})}{1+\mathrm{ST}^{*}}
$$

In order to calculate the measured real coincidence rate, $r$, (with counting losses included), consider that at $t=0$ a fission event occurs producing $v$ neutrons. At a later time, $t$, one of the fission neutrons is detected producing a gate signal which following a delay, $t_{d}$, opens the coincidence gate for a open time, $\mathrm{T}$.

The probability, $P_{c}$, that a coincidence gate signal is generated between $t$ and $t+d t$ is given by:

$\mathbf{P}_{\mathrm{c}}=\mathbf{n}_{\mathrm{c}} \mathrm{dt}$

The probability that the coincidence gate above will be opened by a neutron from the fission at $t=0$ is equal to the ratio of the probability that a neutron from the fission will be detected between $t$ and $t+d t$, $v \varepsilon \eta(t) d t$, to the total probability that any neutron will be detected between $t$ and $t+d t, S d t$, (where $\eta(t)$ is the normalized die-away function for neutrons detected by the detection system). In this case we have written the die away as a general function of $t$ rather than assuming that it follows a simple exponential decay with a die-away constant, $\tau$. The probability that a neutron from the fission at $t=0$ produces a 
coincidence gate between $t$ and $t+d t$, denoted as $P_{g}(t) d t$, would be the product of the probability that a coincidence gate is opened times the probability that the coincidence gate was opened by a neutron from the fission.

$$
P_{g}(t) d t=\frac{v \varepsilon \eta(t) d t}{S d t} n_{c} d t=\frac{v \varepsilon \eta(t)}{1+S T^{*}} d t
$$

For a coincidence gate with an open time of $T$ and a start delay time, $t_{d}$, the number of counts in a gate triggered by a neutron from the fission at time $t$ following the fission with $v$ neutrons produced is denoted as $\mathrm{N}_{\mathrm{v}}$.

$$
N_{v}=\int_{t+t_{d}}^{t+t_{d}+T} \frac{S\left(t^{\prime}\right)}{1+S\left(t^{\prime}\right) \delta t} d t^{\prime}
$$

If $S\left(t^{\prime}\right) \delta t<1$ then Equation (7.14a) can be approximated by:

$$
N_{v}=\int_{t+t_{d}}^{t+t_{d}+T} S\left(t^{\prime}\right)\left[1-S\left(t^{\prime}\right) \delta t\right] d t^{\prime}
$$

$S\left(t^{\prime}\right)$ is the time dependent singles count rate:

$S\left(t^{\prime}\right)=\varepsilon(v-1) \eta\left(t^{\prime}\right)+S$

where $S$ is the time averaged singles rate given by Equation (7.3). Combining Equation (7.15) with Equation (7.14b) and keeping only zero and first order terms in $\varepsilon$, the coincidence count per gate is given by:

$$
N_{v}(t) \approx \varepsilon(v-1)(1-2 S \delta t)\left(\int_{t+t_{d}}^{t+t_{d}+T} \eta\left(t^{\prime}\right) d t^{\prime}\right)+S[1-S \delta t] T
$$

The additive term on the right of Equation (7.16) is the accidental count during this gate window. The product of $P_{g}(t) d t$ times $N_{v}$ is the number of coincidence counts in a gate triggered by a neutron count between $\mathrm{t}$ and $\mathrm{dt}$ due to a fission at $\mathrm{t}=0$.

To obtain the total number of coincidence counts for each fission with $v$ neutrons produced, one must integrate $N_{v} P_{g}(t) d t$ from $t=0$ to $t=\infty$. The integral of the first term in Equation (7.16) is the real coincidence counts per fission, $R_{v}$, and integral of the second term in Equation (7.16) is the accidental counts per fission, $A_{v}$.

The real coincidence counts per fission is

$$
\mathrm{R}_{v}=\frac{v(v-1) \varepsilon^{2}}{\left(1+\mathrm{ST}^{*}\right)(1+2 \mathrm{~S} \delta \mathrm{t})} \int_{0}^{\infty} \mathrm{dt} \eta(\mathrm{t}) \int_{\mathrm{t}+\mathrm{t}_{\mathrm{d}}}^{\mathrm{t}+\mathrm{t}_{\mathrm{d}}+\mathrm{T}} \mathrm{dt} \mathrm{t}^{\prime} \eta\left(\mathrm{t}^{\prime}\right)
$$


and the accidental coincidence counts per fission is:

$$
A_{v}=\frac{\varepsilon v S T}{\left(1+S T^{*}\right)(1+S \delta t)} \int_{0}^{\infty} \eta(t) d t
$$

where the terms $(1-2 S \delta t)$ and $(1-S \delta t)$ in the numerator of Equation 7.17 have been replaced by (1+ $2 S \delta t)$ and $(1+S \delta t)$, respectively, in the denominator. Since $\eta(t)$ is normalized, the integral in Equation (7.18) is equal to 1 and the accidental coincidence count per fission becomes:

$$
\mathrm{A}_{v}=\frac{\varepsilon v S T}{\left(1+S T^{*}\right)(1+\mathrm{S} \delta \mathrm{t})}
$$

Equations 7.17, 7.18, and 7.19 are more appropriate if $S \delta t$ is approaching unity. The average coincidence counts per fission is the weighted sum over the statistical distribution of $v$ neutrons per fission times the coincidence counts per $v$ fission. The real coincidence count per fission, $R_{c f}$ is:

$$
\mathrm{R}_{\mathrm{cf}}=\sum_{v=1} \mathrm{G}_{v} \mathrm{R}_{v}
$$

The accidental coincidence count per fission, $\mathrm{A}_{\mathrm{cf}}$, is:

$$
A_{c f}=\sum_{v=1} G_{v} A_{v}
$$

where $G_{v}$ is the normalized probability that a fission will yield $v$ neutrons.

Substituting the expressions for $R_{v}$ and $A_{v}$ from Equations (7.17) and (7.19), respectively, into Equations (7.20a) and (7.20b) yields:

$$
\begin{aligned}
& \mathrm{R}_{\mathrm{cf}}=\frac{\varepsilon^{2}}{\left(1+\mathrm{ST}^{*}\right)(1+2 \mathrm{~S} \delta \mathrm{t})} \sum_{v=1} \mathrm{G}_{v} v(v-1) \int_{0}^{\infty} \mathrm{dt} \eta(\mathrm{t}) \int_{\mathrm{t}+\mathrm{t}_{\mathrm{d}}}^{\mathrm{t}+\mathrm{t}_{\mathrm{d}}+\mathrm{T}} \mathrm{d} \mathrm{t}^{\prime} \eta\left(\mathrm{t}^{\prime}\right) \\
& \mathrm{A}_{\mathrm{cf}}=\frac{\varepsilon S T}{\left(1+\mathrm{ST}^{*}\right)(1+\mathrm{S} \delta \mathrm{t})} \sum_{v=1} \mathrm{G}_{v} v .
\end{aligned}
$$

The sum in Equation (7.22a) is equal to the average number of neutrons per fission, $v_{a v}$, therefore, Equation (7.22a) reduces to:

$$
A_{c f}=\frac{\varepsilon v_{\mathrm{av}} S T}{\left(1+S T^{*}\right)(1+S \delta \mathrm{t})}
$$

The real coincidence rate (with counting loss included), $r$, is equal to the product of the fission rate, $N_{f}$, given in Equation (7.1) with the real coincidence count per fission, $R_{c f}$, given in Equation (7.21). 


$$
\mathrm{r}=\frac{\lambda_{\mathrm{sf}} \mathrm{N}_{240} \varepsilon^{2}}{\left(1+\mathrm{ST}^{*}\right)(1+2 \mathrm{~S} \delta \mathrm{t})} \sum_{v=1} \mathrm{G}_{v} v(v-1) \int_{0}^{\infty} \mathrm{dt} \eta(\mathrm{t}) \int_{\mathrm{t}+\mathrm{t}_{\mathrm{d}}}^{\mathrm{t} \mathrm{t}_{\mathrm{d}}+\mathrm{T}} \mathrm{dt}^{\prime} \eta\left(\mathrm{t}^{\prime}\right)
$$

The accidental coincidence rate (with counting loss), $\mathrm{a}_{\mathrm{cf}}$, due to gates triggered by fission neutrons is equal to the product of the fission rate, $\mathrm{N}_{\mathrm{f}}$, given in Equation (7.1) with the accidental coincidence count per fission, $\mathrm{A}_{\mathrm{cf}}$, given in Equation (7.22b).

$$
a_{\mathrm{cf}}=\frac{\left.\lambda_{\mathrm{sf}} \mathrm{N}_{240} \varepsilon v_{\mathrm{av}} \mathrm{ST}\right)}{\left(1+\mathrm{ST}^{*}\right)(1+\mathrm{S} \delta \mathrm{t})}
$$

Substituting the F from Equation (7.2) into Equation (7.24a) yields:

$$
\mathrm{a}_{\mathrm{cf}}=\frac{\mathrm{FST}}{\left(1+\mathrm{ST}^{*}\right)(1+\mathrm{S} \delta \mathrm{t})}
$$

In a similar fashion the accidental coincidence rate (with counting loss) due to non-fission triggered gates, $a_{n f}$, can be derived by determining the rate of non-fission triggered coincidence gates times the total coincidence count per gate. The result is:

$$
a_{n f}=\frac{A S T}{\left(1+S T^{*}\right)(1+S \delta t)}
$$

The sum of Equations (7.24) and (7.25) is the total accidental coincidence rate, a:

$$
a=\frac{S^{2} T}{\left(1+S T^{*}\right)(1+S \delta t)}
$$

In Equation (7.26), $\mathrm{F}+\mathrm{A}$ has been replaced by $\mathrm{S}$.

\subsection{Calculating the PAN Response}

There are two phases to calculating the PAN response. First, the measured count data as would be produced during the acquisition phase of a PAN passive measurement on a drum must be obtained. This process is covered in Sections 7.2.1 - 7.2.3 below. Second, the count data must be processed through the sarne analysis procedures as used in the data analysis phase of the PAN analysis program. This is covered in Section 7.2.4.

\subsubsection{Input Parameters for Calculating PAN Response}

Table 7-1 lists the parameters required to calculate the PAN response using the equations presented in Section 7.1. Most of the parameters come directly from the simulated drum measurement data set described in Section 6. The last two parameters, detector efficiency, $\varepsilon$, and normalized die away function, $\eta(t)$, are produced by the MCNP simulation code.

As explained in Section 2, the PAN passive method has two coincidence counting modes. One mode uses the counts coming from the shielded counters and is called the short-gate coincidence mode. The 
other coincidence mode uses counts coming from all detectors (called system or system total) and this coincidence mode is called the long-gate coincidence mode. Parameters al-a11 below apply equally to both coincidence modes, but parameters a12-a15 are specific to whichever mode is being calculated. The approach described in the discussions which follow is equally applicable to both coincidence modes and for the sake of brevity the equations have been written with subscripts indicating mode implied rather than explicitly written.

Table 7-1. Input parameters for calculating the PAN response.

\begin{tabular}{|c|l|}
\hline Parameter & \\
\hline $\mathrm{a} 1$ & Count time, $\mathrm{t}_{\mathrm{c}}$ \\
\hline $\mathrm{a} 2$ & Mass of weapons grade $\mathrm{Pu}, \mathrm{M}_{\mathrm{wg}}$ \\
\hline $\mathrm{a} 3$ & Mass fraction of $\mathrm{Pu}-240, \mathrm{MF}_{40}$ \\
\hline $\mathrm{a} 4$ & Mass fraction of $\mathrm{Pu}$ in chunks \\
\hline $\mathrm{a} 5$ & Mass fraction of Pu in fines \\
\hline $\mathrm{a} 6$ & Spatial distribution of Pu chunks \\
\hline $\mathrm{a} 7$ & Spatial distribution of Pu fines \\
\hline $\mathrm{a} 8$ & Fill height \\
\hline $\mathrm{a} 9$ & Average matrix density in the drum \\
\hline $\mathrm{a} 10$ & Spatial distribution of matrix density \\
\hline $\mathrm{a} 11$ & Elemental composition of matrix \\
\hline $\mathrm{a} 12$ & Uncorrelated neutron; i.e., $(\alpha, \mathrm{n})$, singles count rate, $\left(\mathrm{A}_{\mathrm{n}}\right)_{\mathrm{sh}},\left(\mathrm{A}_{\mathrm{n}}\right)_{\mathrm{sy}}$ \\
\hline $\mathrm{a} 13$ & Background singles count rate, $\left(\mathrm{B}_{\mathrm{s}}\right)_{\mathrm{sh}},\left(\mathrm{B}_{\mathrm{s}}\right)_{\mathrm{sy}}$ \\
\hline $\mathrm{a} 14$ & Background coincidence count rate, $\left(\mathrm{B}_{\mathrm{c}}\right)_{\mathrm{sg}},\left(\mathrm{B}_{\mathrm{c}}\right)_{\mathrm{gs}}$ \\
\hline $\mathrm{a} 15$ & Detector efficiency, $\varepsilon_{\mathrm{sh}}, \varepsilon_{\mathrm{sy}}{ }^{1}$ \\
\hline $\mathrm{a} 16$ & Normalized die-away function, $\eta_{\mathrm{sh}}(\mathrm{t}), \eta_{\mathrm{sy}}(\mathrm{t})$ \\
\hline
\end{tabular}

1. For each case identified in the statistical set, items a2-a11 are used to calculate, using MCNP (see Section 7.2.2), the detector efficiency (number of counts per source neutron) for the shielded detectors, $\varepsilon_{\mathrm{sh}}$, and for the shielded plus bare (system) detectors, $\varepsilon_{\text {sy }}$

\subsubsection{Monte Carlo Neutron Photon Calculation}

The calculation approach used in this evaluation involves the use of the Monte Carlo Neutron Photon (MCNP) transport code developed at Los Alamos National Laboratory (Briesmeiser, 1986) and a model of the SWEPP PAN assay system developed by Yoon (1993). The code and model have been verified by comparisons of the calculated responses with measurements using the PAN assay system and calibration drums designed to represent actual waste forms. Section 8 of this report provides comparisons of measured and simulated results for the glass waste content codes.

The MCNP model for this application is set up to provide detector efficiencies for the shielded and system (shielded plus bare) detector banks; i.e., the number of neutron events in the detector bank per source neutron. In addition it also provides a time history of the neutron events in each detector bank following the introduction of a source neutron. However, the time history requires considerably longer computer running times and was not performed for all cases. Nevertheless, enough time histories were generated to determine that, at least for glass waste, the normalized coincidence response integrals as contained in Equation 7.23 are not sensitive to the variations in the model. 
The model of the detector enclosure, detector packages, etc. is the result of a dedicated effort to duplicate the system exactly in the "as built" configuration. In order to do this the PAN system was partially dismantled to identify the precise configuration of this particular system.

Two models were used in the MCNP calculations: one for the glass waste calibration drum simulations and the other for the cases simulating actual glass waste drums. The PAN detector enclosure, detectors, drum and 90 mil polyethylene liner were modeled exactly the same in both the calibration model and waste drum model. The differences between the two models are in the drum contents.

The model for the calibration drum was divided into 3 elevation levels, each containing four angular subzones. The zonal composition and density are varied to represent the heterogeneous nature of glass waste in the calibration drum. The sample access tubes (used for placing sources in the drum--see Section 8) are also modeled. The model for the simulated waste drums is very similar to the calibration drum except that there are no sample access tubes and the zones are divided into 8 elevations with 3 radial zones per elevation. For each simulated waste drum, the elemental inventory for each zone is modeled to approximate those of a real waste drum from the SWEPP inventory using the methods described in Section 6.

In actual measurements the drum would be rotating. To simulate rotation, four computer runs were completed for each simulated drum. For each of the four runs, the drum contents are rotated $90^{\circ}$ from the orientation of the previous run. The final results for each case is the average of the four runs. An exception to this procedure occurs for cases where there are no chunks of plutonium specified. In this situation, because the fines component of the $\mathrm{Pu}$ source and the matrix specification are already cylindrically symmetric, only one computer run is necessary. The length of a computer run is set so that a Monte Carlo statistical error of $2 \%$ is achieved for the shielded detector bank efficiency. The corresponding statistical error for the system detector bank efficiency is less than $1 \%$.

\subsubsection{Calculation Procedure for PAN Passive Response}

The following parameters constitute the output data from acquisition phase of a PAN passive measurement. These data constitute the input to the SWEPP PAN data analysis program. This program is referred to as "NEUT2" (old analysis program) or as "SWEPP Assay System" (new analysis program).

Count Time. Typically the count time is $200 \mathrm{sec}$ for waste drum assay; however for the calibration measurements using the glass waste calibration drum, the count time is either $600 \mathrm{sec}$ (for content code 440 ) or $400 \mathrm{sec}$ (for content code 442). The PAN acquisition monitors the count time by a scalar count of the pulses from a $1-\mathrm{kHz}$ oscillator and so count time monitor data are the number of $1-\mathrm{kHz}$ counts and the count time is determined by dividing the $1-\mathrm{kHz}$ counts by the frequency of the oscillator. Typically the measured count time deviates from the set time by less than a second. In the simulation the set count time is used to avoid having to simulate the count time measurement.

Raw Singles Count. The raw singles count and the standard deviation based on counting statistics for the shielded, $\left(\mathrm{S}_{\mathrm{r}}\right)_{\mathrm{sh}}$, and the system, $\left(\mathrm{S}_{\mathrm{r}}\right)_{s y}$, are measurements calculated using the following steps. To achieve a simulated singles count with deviations consistent with counting statistics, the terms true singles count and standard deviation are calculated in steps 1-7 and the simulated measured singles count is calculated in step 8 below. (Note: These steps are the same for both the shielded and system responses and the subscripts "sh" and "sy" are implied depending on the response being calculated.) 
1. Use Equation (7.27), $\mathrm{M}_{\mathrm{wg}}$ (a2) and $\mathrm{MF}_{40}$ (a3) to calculate $\mathrm{N}_{240}$.

$\mathrm{N}_{240}=\frac{\mathrm{MF}_{40} \mathrm{M}_{\mathrm{wg}}(\mathrm{g}) 6.022 \times 10^{23}}{240.054}$

$v_{a v}=2.16$ neutrons per spontaneous fission of $\mathrm{Pu}-240$

$$
\lambda_{\mathrm{sf}}\left(\mathrm{s}^{-1}\right)=\frac{0.69315}{\left(1.16 \times 10^{11} \mathrm{yr}\right)\left(3.156 \times 10^{7} \mathrm{~s} / \mathrm{yr}\right)}=1.893 \times 10^{-19} \mathrm{~s}^{-1}
$$

The spontaneous fission half life and the average number of neutrons per spontaneous fission for Pu-240 are from NUREG-CR-5550. Using these constants the fission neutron emission rate per gram of $\mathrm{Pu}-240$ is $1026 \mathrm{n} / \mathrm{s}$.

2. Use Equation (7.2), $\mathrm{N}_{240}, \lambda_{\mathrm{sf}}, \mathrm{v}_{\mathrm{av}}$, and $\varepsilon$ (a15) to calculate $\mathrm{F}$.

3. Use Equation (7.28), $A_{n}(a 12)$, and $B_{s}(a 13)$ to calculate $A$.

$$
A=A_{n}-F+B_{s}
$$

4. Use Equation (7.3), $\mathrm{F}$ and $\mathrm{A}$ to calculate $\mathrm{S}$.

5. Assume $\delta t=5 \mu \mathrm{s}$, use Equation (7.4) and $S$ to calculate $n$.

6. Use Equation (7.29), $\mathrm{t}_{\mathrm{c}}$ (a1) and $\mathrm{n}$ to calculate the true raw singles count (no counting statistics), $\left(\mathrm{S}_{\mathrm{r}}\right)^{*}$.

$\left(\mathrm{S}_{\mathrm{r}}\right)^{*}=\mathrm{nt}_{\mathrm{c}}$

7. Use Equation (7.30), to calculate the standard deviation, $\left(\sigma_{\mathrm{sr}}\right)^{*}$, of the true raw singles count, $\left(\mathrm{S}_{\mathrm{r}}\right)^{*}$.

$$
\left(\sigma_{\mathrm{sr}}\right)^{*}=\sqrt{\left(\mathrm{S}_{\mathrm{r}}\right)^{*}}
$$

8. Use Equation (7.31), to calculate the simulated measured raw singles count (with counting statistics), $\mathbf{S}_{\mathbf{r}}$.

$S_{\mathrm{r}}=\left(\mathrm{S}_{\mathrm{r}}\right)^{*}+\delta_{\mathrm{s}}$

where: $\delta_{\mathrm{s}}$ is a statistical variant based on a Poisson distribution with a standard deviation $\left(\sigma_{\mathrm{sr}}\right)^{*}$.

Coincidence Gate Time. For the short-gate coincidence, the gate time, $T_{\text {sg }}$, is set in the PAN system at $35 \mu \mathrm{s}$ and for the long-gate coincidence, the gate time, $T_{1 g}$, is set in the PAN system at $250 \mu$ s. These gate times are also monitored by the PAN acquisition system counting pulses in a short-gate scalar and in a long-gate scalar from a $1 \mathrm{MHz}$ oscillator during the time the corresponding gate is opened for coincidence counting. The measured gate times have been shown to be very consistent with the specified 
values and variations in gate times are not significant in determining the deviations in the system response. Therefore, in this simulation the specified gate times have been used.

Number of Coincidence Gates. The delay following a detector pulse before the opening of the corresponding coincidence gate, $t_{d}$, has been set in the PAN system at $6 \mu$ s for both the short-gate and long-gate coincidence modes. It is assumed that the typical detector dead time for processing a pulse (i.e., $5 \mu$ s) can be used to determine the small counting loss in single pulse acquisition. The following steps are used to calculate the number of coincidence gates for the short gate, $\left(\mathrm{N}_{\mathrm{c}}\right)_{\mathrm{sg}}$, and for the long gate, $\left(\mathrm{N}_{\mathrm{c}}\right)_{\mathrm{lg}}$. Once again the subscripts "sg" and "lg" are implied.

1. Use Equation (7.9), $\mathrm{T}, \mathrm{t}_{\mathrm{d}}, \delta \mathrm{t}$, and $\mathrm{S}$ to calculate $\mathrm{n}_{\mathrm{c}}$.

2. Use Equation (7.32), $\mathbf{n}_{\mathrm{c}}$, and $\mathrm{t}_{\mathrm{c}}(\mathrm{a} 1)$ to calculate number of coincidence gates, $\mathbf{N}_{\mathrm{c}}$.

$$
\mathrm{N}_{\mathrm{c}}=\mathrm{n}_{\mathrm{c}} \mathrm{t}_{\mathrm{c}}
$$

Raw Coincidence Count and Standard Deviation. In the simulation, to achieve the raw coincidence count with counting statistical deviations included the terms true raw coincidence count and standard deviation are calculated in steps 1-4 below. Then the true values are used to calculate the simulated measured raw coincidence count and standard deviation (step 5).

1. Use Equation (7.23), Equation (7.6), $\lambda_{s f}, N_{240}, \varepsilon(a 15), S, T, T^{*}, \delta t, t_{d}$ to calculate the real coincidence rate, $r$, where for the short-gate coincidence mode:

$$
\int_{0}^{\infty} d t \eta(t) \int_{t+t_{d}}^{t+t_{d}+T} d t^{\prime} \eta\left(t^{\prime}\right)=.2853 \text { for content codes } 440 \text { and } 441 \text { and } .2815 \text { for content code } 442 \text {. }
$$

Similarly for the long-gate coincidence mode:

$$
\int_{0}^{\infty} d t \eta(t) \int_{t+t_{d}}^{t+t_{d}+T} d t^{\prime} \eta\left(t^{\prime}\right)=.3746 \text { for content codes } 440 \text { and } 441 \text { and } .3786 \text { for content code } 442 .
$$

2. Use Equation (7.26), S, T, $\mathrm{T}^{*}$, and $\delta \mathrm{t}$ to calculate the accidental rate, a.

3. Use Equation (7.33), $r$ (Equation 7.23), a (Equation 7.26), $t_{c}(a 1)$, and $B_{c}$ (a13) to calculate true raw coincidence count (without counting statistics included), $\left(\mathrm{C}_{\mathrm{r}}\right)^{*}$.

$$
\left(C_{r}\right)^{*}=\left(r+a+B_{c}\right) t_{c}
$$

4. Use Equation (7.34), to calculate the true standard deviation $\left(\sigma_{\mathrm{cr}}\right)^{*}$.

$$
\left(\sigma_{\mathrm{cr}}\right)^{*}=\sqrt{\left(\mathrm{C}_{\mathrm{r}}\right)^{*}}
$$

5. Use Equation (7.35), the true raw coincidence rate, $\left(C_{\mathrm{r}}\right)^{*}$, and its standard deviation, $\left(\sigma_{\mathrm{cr}}\right)^{*}$, to calculate the simulated measured raw coincidence rate, $\mathrm{C}_{\mathrm{r}}$. 


$$
\mathrm{C}_{\mathrm{r}}=\left(\mathrm{C}_{\mathrm{r}}\right)^{*}+\delta_{\mathrm{c}}
$$

where $\delta_{c}$ is a statistical variant based on the Poisson distribution with a standard deviation, $\left(\sigma_{\mathrm{cr}}\right)^{*}$.

\subsubsection{Simulation of PAN Data Analysis Procedure}

The equations listed below are from the SWEPP Assay System analysis program description report (Matthews, 1993) and are the same as used in the NEUT2 analysis program with the exception that the calculation of live time in the SWEPP Assay System program correctly accounts for lost time due to the coincidence gate delay, $t_{d}$, whereas there was a mistake in the NEUT2 algorithm. A computer program was written to implement these calculations for the simulated glass waste drum measurements. The program is described in detail in Appendix A.

PAN Analysis Procedure Input Parameters. The parameters required for the PAN analysis calculations are listed in Table 7-2.

Table 7-2. Parameters required for PAN analysis calculations.

\begin{tabular}{|c|c|}
\hline Parameter & Description \\
\hline b1 & Count time, $t_{c}$ \\
\hline b2 & Raw singles counts, $\left(\mathrm{S}_{\mathrm{r}}\right)_{\mathrm{sh}},\left(\mathrm{S}_{\mathrm{r}}\right)_{\mathrm{sv}}$ \\
\hline b3 & Coincidence gate times, $T_{s g}, T_{l g}$ \\
\hline b4 & Number of coincidence gates, $\left(\mathrm{N}_{\mathrm{c}}\right)_{\mathrm{sg}},\left(\mathrm{N}_{\mathrm{c}}\right)_{\mathrm{gg}}$ \\
\hline b5 & Raw coincidence counts, $\left(C_{r}\right)_{s g},\left(C_{r}\right)_{l g}$ \\
\hline b6 & Raw coincidence count standard deviations, $\left(\sigma_{\mathrm{c}}\right)_{\mathrm{sg}},\left(\sigma_{\mathrm{c}}\right)_{\mathrm{gg}}$ \\
\hline b7 & Mass fraction of $\mathrm{Pu}-240, \mathrm{MF}_{40}$ (set equal to 0.0582 ) \\
\hline b8 & Background singles count rate, $\left(\mathrm{B}_{\mathrm{s}}\right)_{\mathrm{sh}},\left(\mathrm{B}_{\mathrm{s}}\right)_{\mathrm{sv}}$ (for the daily background drum) \\
\hline b9 & Background coincidence count rate, $\left(B_{c}\right)_{s h},\left(B_{c}\right)_{s v}$ (for the daily background drum) \\
\hline $\mathrm{b} 10$ & Background coincidence rate error, $\left(\sigma_{b c}\right)_{s g},\left(\sigma_{b c}\right)_{l g}$ (for the daily background drum) \\
\hline b11 & Absorber index, AI \\
\hline
\end{tabular}

Simulation of the PAN Analysis of the Passive Response Data. The general steps in the PAN analysis simulation are:

1. Using the input parameters from Table 7-2, the Pu mass as determined from the short-gate coincidence data (referred to as the short-gate Pu mass) and the Pu mass as determined from the long-gate coincidence data (referred to as the long-gate Pu mass) are calculated separately using exact duplicate algorithms of those found in the current version of the PAN data analysis program.

2. Based on the selection criteria that exists in the current version the PAN data analysis program, the "passive plutonium mass" is selected from either the short-gate $\mathrm{Pu}$ mass or the long-gate $\mathrm{Pu}$ mass. For the glass waste and many other content codes the selection criterion is based on which of the two Pu masses has the smallest relative error based on counting statistics alone.

As has been the case in determining the simulated PAN response data above, the approach shown below is the same for both the shielded singles (short-gate) coincidence data and the system singles (long-gate) coincidence data and therefore the subscripts "sh", "sg", "sy", "lg" are implied in the equations shown. 
1. Use Equation (7.36) to compute total gate times, $\left(\mathrm{T}_{\mathrm{g}}\right)_{\mathrm{sg}},\left(\mathrm{T}_{\mathrm{g}}\right)_{\mathrm{gg}}$. Each of these gate times is the total time that the respective coincidence gate is opened over the course of the measurement and is determined from the number of coincidence gates, $\mathrm{N}_{c}$, and the open time per gate, $\mathrm{T}$.

$\mathrm{T}_{\mathrm{g}}=\mathrm{TN}_{\mathrm{c}}$

2. Use Equation (7.37) to compute total live times, $\left(T_{1}\right)_{s g},\left(T_{1}\right)_{\mathrm{lg}}$. Each of these live times is calculated from the total count time, $t_{c}$, the total gate time, $T_{g}$, for the respective coincidence unit and the delay time per gate, $t_{d}$.

$T_{1}=t_{c}-T_{g}-N_{c} t_{d}$

3. Use Equation (7.38) to compute the gross coincidence rates, $\left(\mathrm{C}_{\mathrm{g}}\right)_{\mathrm{sg}},\left(\mathrm{C}_{\mathrm{g}}\right)_{\mathrm{lg}}$. Each of these coincidence rates is calculated from the respective raw coincidence count, $\mathrm{C}_{\mathrm{r}}$, the respective singles count rate, $n$, the respective total gate time, $T_{g}$, and the respective live time, $T_{1}$. The result is a coincidence rate which has been corrected for accidental coincidence events.

$$
\mathrm{C}_{\mathrm{g}}=\frac{\mathrm{C}_{\mathrm{r}}-\mathrm{nT}_{\mathrm{g}}}{\mathrm{T}_{\mathrm{l}}}
$$

4. Use Equation (7.39) to compute the measured coincidence rates, $\left(C_{m}\right)_{s g},\left(C_{m}\right)_{\mathrm{lg}}$. Each of these rates is calculated from the respective gross coincidence rate, $C_{g}$, and the respective background coincidence rate, $B_{c}$, (b9). The result is the measured coincidence rate which has now been corrected for both accidental coincidence events and background coincidence events.

$C_{m}=C_{g}-B_{c}$

5. Use Equation (7.40), $S_{r}, t_{c}$, and $B_{s}(b 8)$ to compute the net singles count rates, $\left(S_{m}\right)_{s h},\left(S_{m}\right)_{s y}$.

$$
S_{m}=\frac{S_{r}}{t_{c}}-B_{s}
$$

6. Use Equation (7.41), $\mathrm{S}_{\mathrm{r}}$, and $\mathrm{t}_{\mathrm{c}}$ to compute the raw singles rate standard deviations, $\left(\sigma_{\mathrm{n}}\right)_{\mathrm{sh}},\left(\sigma_{\mathrm{n}}\right)_{\mathrm{sy}}$. As calculated here, these values are based on counting statistics only.

$$
\sigma_{\mathrm{n}}=\frac{1}{\mathrm{t}_{\mathrm{c}}} \sqrt{\mathrm{S}_{\mathrm{r}}}
$$

7. Use Equation (7.42), $T_{1}, C_{r}, \sigma_{n}$, and $T_{g}$ to compute the gross coincidence rate standard deviations, $\left(\sigma_{\mathrm{cg}}\right)_{\mathrm{sg}},\left(\sigma_{\mathrm{cg}}\right)_{\mathrm{lg}}$.

$$
\sigma_{c g}=\frac{1}{T_{1}} \sqrt{C_{r}+\left(\sigma_{n} T_{g}\right)^{2}}
$$

8. Use Equation (7.43) to compute the measured coincidence rate standard deviations, $\left(\sigma_{\mathrm{cm}}\right)_{\mathrm{sg}}$, $\left(\sigma_{\mathrm{cm}}\right)_{\mathrm{lg}}$. 
$\sigma_{\mathrm{cm}}=\frac{1}{\mathrm{~T}_{1}} \sqrt{\left(\sigma_{\mathrm{cg}}\right)^{2}+\left(\sigma_{\mathrm{bc}}\right)^{2}}$

9. Compute the moderator index, MI. The moderator index is the same for both the short-gate and long-gate coincidence units.

If the net shielded singles rate, $\left(S_{\mathrm{m}}\right)_{\mathrm{sh}}$, is less than or equal to 10 counts per second, set the Moderator Index, MI, to 0.15 .

If the net system singles rate, $\left(\mathrm{S}_{\mathrm{m}}\right)_{\mathrm{sy}}$, is less than or equal to 0.0 counts per second, set the Moderator Index, MI, to 0.0 .

Otherwise use Equation (7.44):

$\mathrm{MI}=\left[1-4.42 \frac{\left(\mathrm{S}_{\mathrm{m}}\right)_{\mathrm{sh}}}{\left(\mathrm{S}_{\mathrm{m}}\right)_{\mathrm{sy}}}\right][1.04+0.2924 \ln (\mathrm{AI})]$

10. Compute the isotopic correction factor, $\mathrm{CF}_{\mathrm{I}}$.

If $\mathrm{MF}_{40}$ is less than 0.01 or greater than 0.99 , set $\mathrm{CF}_{\mathrm{I}}$ equal to 1.0 .

Otherwise use Equation (7.45):

$\mathrm{CF}_{\mathrm{I}}=\frac{\left(\mathrm{MF}_{40}\right)_{\mathrm{wgPu}}}{\mathrm{MF}_{40}}$

where the default value for $\left(\mathrm{MF}_{40}\right)_{\mathrm{wgPu}}$ is equal to 0.0582 .

11. Use Equation (7.46) to compute the short-gate coincidence correction factor, $\mathrm{CF}_{\mathrm{sg}}$.

$$
\mathrm{CF}_{\mathrm{sg}}=\left(\frac{0.8092}{1-\mathrm{MI}}+0.2337\right)^{2}
$$

12. Use Equation (7.47) to compute the long-gate coincidence correction factor, $\mathrm{CF}_{\mathrm{lg}}$.

$$
\mathrm{CF}_{\mathrm{lg}}=\left(\frac{0.5967}{1-\mathrm{MI}}+0.4187\right)^{2}
$$

13. Use Equation (7.48) to compute the short-gate Pu mass, $\left(\mathrm{M}_{\mathrm{Pu}}\right)_{\mathrm{sg}}$.

$$
\left(\mathrm{M}_{\mathrm{pu}}\right)_{\mathrm{sg}}=28.1 \mathrm{CF}_{\mathrm{sg}} \mathrm{CF}_{\mathrm{I}}\left(\mathrm{C}_{\mathrm{m}}\right)_{\mathrm{sg}}
$$

14. Use Equation (7.49) to compute the long-gate Pu mass, $\left(\mathbf{M}_{\mathrm{pu}}\right)_{\mathrm{lg}}$.

$$
\left(\mathrm{M}_{\mathrm{pu}}\right)_{\mathrm{lg}}=1.28 \mathrm{CF}_{\mathrm{lg}} \mathrm{CF}_{\mathrm{I}}\left(\mathrm{C}_{\mathrm{m}}\right)_{\mathrm{lg}}
$$


15. Use Equation (7.50) to compute the standard deviation in the short-gate Pu mass, $\left(\sigma_{\mathrm{Pu}}\right)_{\mathrm{sg}}$.

Note: the computed standard deviation includes only the statistical errors due to counting.

$$
\left(\sigma_{\mathrm{Pu}}\right)_{\mathrm{sg}}=\frac{\left(\sigma_{\mathrm{cm}}\right)_{\mathrm{sg}}}{\left(\mathrm{C}_{\mathrm{m}}\right)_{\mathrm{sg}}}\left(\mathrm{M}_{\mathrm{Pu}}\right)_{\mathrm{sg}}
$$

16. Use Equation (7.51) to compute the standard deviation in the long-gate $\mathrm{Pu}$ mass, $\left(\sigma_{\mathrm{Pu}}\right)_{\mathrm{g}}$.

Note: the computed standard deviation includes only the statistical errors due to counting.

$$
\left(\sigma_{\mathrm{Pu}}\right)_{\mathrm{lg}}=\frac{\left(\sigma_{\mathrm{cm}}\right)_{\mathrm{lg}}}{\left(\mathrm{C}_{\mathrm{m}}\right)_{\mathrm{lg}}}\left(\mathrm{M}_{\mathrm{Pu}}\right)_{\mathrm{lg}}
$$

17. Select the passive plutonium mass.

If the absolute value of the relative error for the short-gate coincidence rate is less than the absolute value of the relative error for the long-gate coincidence rate, i.e.

$$
\left|\frac{\left(\sigma_{\mathrm{cm}}\right)_{\mathrm{sg}}}{\left(C_{\mathrm{m}}\right)_{\mathrm{sg}}}\right| \leq\left|\frac{\left(\sigma_{\mathrm{cm}}\right)_{\mathrm{lg}}}{\left(C_{\mathrm{m}}\right)_{\mathrm{lg}}}\right|,
$$

the passive plutonium mass and its error are set equal to the plutonium mass and its error from the short-gate coincidence unit:

$$
\mathbf{M}_{\mathrm{pu}}=\left(\mathrm{M}_{\mathrm{pu}}\right)_{\mathrm{sg}} \quad \text { and } \quad \sigma_{\mathrm{Pu}}=\left(\sigma_{\mathrm{Pu}}\right)_{\mathrm{sg}} .
$$

Otherwise, the passive plutonium mass and its error are set equal to the plutonium mass and its error from the coincidence system:

$$
\mathrm{M}_{\mathrm{pu}}=\left(\mathrm{M}_{\mathrm{pu}}\right)_{\mathrm{lg}} \quad \text { and } \quad \sigma_{\mathrm{Pu}}=\left(\sigma_{\mathrm{Pu}}\right)_{\mathrm{lg}}
$$

The passive plutonium mass and its error as prescribed by either Equation (7.53) or Equation (7.54) are the values presented in the current version of the PAN assay report. These values are used to compared against the known plutonium mass (a2) to assess the total uncertainty of the PAN assay system. Given the plutonium mass (passive) such quantities as total alpha activity, alpha concentration, fissile gram equivalent and thermal power can be derived. The uncertainties for derived quantities can then be propagated using the total uncertainty of the plutonium mass value determined from this method combined with uncertainties assigned to the mass fractions and other parameters. If the SWEPP gamma spectrometer mass ratio values are being used to obtain the derived quantities then the uncertainties associated with the mass ratios would be included in the propagation of the total uncertainties in the derived quantities. 


\section{CALIBRATION DRUM BENCHMARK RESULTS}

To validate the computer modeling process used for simulating the PAN system results, simulations of actual measurements on two glass waste calibration drums were performed. One calibration drum simulated code 440 (glass vials) waste while the other simulated code 442 waste (leached raschig rings). Since the code 441 waste is essentially the same as code 442 waste (except that the raschig rings are unleached) it was not simulated in the calibration runs.

By comparing the actual to simulated measurement results for the controlled calibration configurations, the ability of the simulation to approximate the PAN system performance can be evaluated. The primary analysis of concern is the comparison of the final $\mathrm{Pu}$ gram quantities reported by the actual and simulated PAN measurements. Various statistical significance tests are utilized in the comparisons. Exact p-values are generally given for each test. References in the discussion to effects being statistically significant refer to cases with p-values less than .05 .

Matrix configuration and related measurement parameters for the calibration drum simulations followed the same general approach as that used for the waste drum simulations. A few minor changes were made to accommodate the different assumptions that can reasonably be made about calibration vs. waste drums and the different types of data that are available. One such difference, described in detail in Section 6, involved the distributions used to generate the absorber index values. Another difference occurred in the generation of background count values. For waste drum simulations, it is necessary to generate background counts for both the waste drum measurement and its associated daily background drum measurement. In the simulations of the actual calibration drum runs however, it is only necessary to simulate the background counts associated with the calibration drum measurement since the actual background counts for the daily background drum measurements can be used. Using the actual values for the daily background drum measurements allows for a more accurate assessment of the ability of the simulation process to mimic the PAN system performance by removing a source of variability extraneous to the assessment of the PAN simulation (i.e., variability in the daily background drum values).

\subsection{Description of the Calibration Drums and Source Configurations for the Calibration Drum Runs}

\subsubsection{Content Code 440 Calibration Drum}

The content code 440 calibration drum is described in detail in Becker (1993b). It is composed of a 208liter ( 55 gallon) drum, a 90-mil rigid polyethylene liner, 22 1-gallon polyethylene jars, 2112 Pyrex glass vials (12 gram per vile) and two Styrofoam support rings. These components are arranged in the following configuration (Becker, 1993b):

There are two types of polyethylene jars used in the content code 440 calibration drum; i.e., source jars and non-source jars. The source jars are described below. Each nonsource polyethylene jar is packed with 96 Pyrex glass vials and the jars are arranged in the 90 mil polyethylene liner in three layers. In each of the bottom two layers the jars are positioned vertically with eight around the periphery and one in the center. In the space between the outer jars and the center jar there is a Styrofoam support ring for each layer. In the topmost layer there are only four jars laying on their sides. The 90-mil liner with its contents are placed inside the 208 liter drum. 
In contrast to other calibration drums, the content code 440 calibration drum has no source insertion tubes with which to externally introduce source material into the drum. For use in the content code 440 calibration drum, a number of source jars were constructed. In a source jar there is a center Styrofoam cylinder into which the source material (usually plutonium foils) are placed and held in position. The remainder of the source jar volume is filled with 48 Pyrex glass vials. The source jars are placed in the calibration drum at specified locations such that they replace the non-source jars at those locations and the total number of jars remains fixed at 22 .

A total of 45 runs with the code 440 calibration drum runs were simulated. Source quantities varied from $2 \mathrm{~g}$ to $129 \mathrm{~g} \mathrm{Pu}$. Source positions were varied both radially and vertically between the runs.

\subsubsection{Content Code 442 Calibration Drum}

The content code 442 calibration drum is described in detail in Becker (1992). It is constructed of a 208 liter (55-gallon) drum, 90-mil polyethylene liner, two 4-mil polyethylene drum bags, $6.5 \mathrm{~kg}$ vermiculite, two fiber pack containers, one 30-mil vinyl bag and two 10 -mil polyethylene bags per fiber pack and $20 \mathrm{~kg}$ of raschig rings per fiber pack. These components are arranged in the following configuration (Becker, 1992):

The raschig rings are contained in double wrapped 10-mil polyethylene bags which are contained in a 30-mil heat sealed vinyl bag which is placed inside a fiber pack. There are two fiber packs per 208-liter drum which are stacked one on top of the other. Vermiculite surrounds the two fiber packs to a height which on average extends to the top of the upper fiber pack. The vermiculite and the two fiber packs are subsequently contained in two polyethylene drum bags which line the 90 -mil liner. The 90 -mil liner sets inside the 208-liter drum.

A set of three vertical source insertion parallel pipeds with exterior access via the drum top surface were installed into the drum through the matrix assembly described above. Inside the insertion parallel pipeds are the source insertion assemblies (boxes) into which the prescribed combination of sources and raschig rings are loaded. The source insertion assemblies are located radially at the drum centerline, the drum mid-radius and the drum outer edge.

A total of 27 runs with the code 442 calibration drum runs were simulated. Source quantities varied from approximately $3 \mathrm{~g}$ to $193 \mathrm{~g} \mathrm{Pu}$. Source positions were varied both radially and vertically between the runs.

\subsection{Bias Adjustment}

The initial simulation results for the glass calibration drum measurements showed a high degree of correlation with the actual PAN system results. The Pearson's $r$ correlation coefficient for the long gate mass values was .994 . For the short gate mass values the correlation was .991. However, a regression analysis of the data indicated some bias was present in the simulations. Regressing the actual PAN measurements on the simulated results produced the following equations:

actual PAN measured long gate mass $=-.0784+1.040$ (simulated PAN measured long gate mass)

actual PAN measured short gate mass $=1.671+1.195$ (simulated PAN measured short gate mass). 
The intercepts from the regression equations represents the amount of constant bias in the simulation process while the slopes are indicative of relative bias. (If there were no bias, the intercept would be zero, and the slope 1.0.) To compensate for these bias relationships, the above regression equations were applied to the raw output from the PAN simulations (for both the calibration and waste drum simulations) to produce adjusted results. All analyses in the remainder of this report are based on these adjusted values.

\subsection{Simulated vs. Actual PAN System Reported Pu Quantities}

The simulated and actual PAN system calculated Pu quantities for each of the glass calibration drum simulations are given in Appendix C (Table C-1). Both the long gate and short gate results are shown. In the simulated and actual PAN data for the calibration drums, the long gate mass was the selected mass (i.e., the mass chosen by the PAN algorithm to be the reported mass value) for all cases. However, the short gate mass was chosen in some of the waste drum simulations. So both the long gate and short gate calibration results are relevant as far as establishing the validity of the simulation process.

\subsubsection{Long Gate Measurement Results}

The actual vs. simulated PAN long gate results for the glass waste calibration drums are plotted in Figure 8-1. The line in the graph indicates where the data would fall if there were perfect agreement of the simulated and actual PAN long gate values. Note that because of the bias adjustment described above, the values should fall about this line on average. However, the adjustment does not guarantee that the agreement will be equally good over all ranges of configurations, nor that the range of deviations from the line will be small.

With the exception of a single outlier, all the data in Figure 8-1 fall closely about the line of perfect agreement. This indicates that the long gate mass modeling process works well over the entire range of $\mathrm{Pu}$ quantities considered. (No specific cause for the one outlier was determined.) The absolute deviations from the line of perfect agreement increase with increasing Pu quantity. However, on a relative basis, the trend is reversed as is indicated in Figure 8-2 which plots the relative percent error in the simulated vs. actual long gate values. The variability and magnitude of the relative error shows a decreasing trend as the quantity of Pu increases. This trend, and in fact most of the observed variability, is primarily due to the random error associated with the Poisson counting statistics in the measurement process. This random error exists for both the actual PAN measurements and the simulated results. By appropriately combining the reported counting errors for the simulated and actual measurements, bounds on the amount of variability to be expected due to counting error alone can be estimated. One such set of bounds is plotted as the curved lines in Figure 8-2.

Specifically, the plotted bounds were calculated as follows:

1. For each calibration drum obtain the standard deviation (due to counting statistics) of the actual PAN $\mathrm{Pu}$ reported measurement, $\mathrm{s}_{\mathrm{a}}$, and the standard deviation for the simulated PAN Pu measurement, $\mathrm{s}_{\mathrm{s}}$, as described earlier.

2. Calculate the standard deviation for the difference, $s_{d}$, in the two measurements (assuming independence) as:

$$
s_{d}=\sqrt{s_{a}^{2}+s_{s}^{2}}
$$


3. Calculate the percent relative standard deviation of the difference as:

$$
\% \text { rsd }=100 \frac{s_{d}}{\text { actual PAN Pu }}
$$

4. Calculate approximate $95 \%$ upper and lower relative percent error bounds by taking $\pm(2)(\%$ rsd).

5. Apply a smoothing procedure to the resulting values for the upper and lower bounds to obtain the curves in the plot. The particular smoothing method used is not critical.

Again these bounds indicate the extent to which the simulated vs. actual measurements can be expected to vary simply due to the uncertainty inherent in the Poisson counting processes. The bounds as plotted assume zero bias in the simulation process (i.e., are centered around the zero error line). That most values in the plot fall within or near these bounds indicates that the variations in simulation results are consistent with what was expected due to random variability in the counts. That is, there is little evidence of excess deviation. The pattern of the deviations does indicate that at the lower mass levels there is a slight tendency for the simulation process to overestimate the PAN measurement results. The effect of this positive bias on the overall uncertainty evaluation process is minor, as indicated by the close agreement of uncertainty measures obtained from the simulated and actual PAN measurements. These measures are discussed in the next section.

\subsubsection{Short Gate Measurement Results}

Figure 8-3 plots the actual vs. simulated PAN results for the calibration drum short gate mass measurements. As with the long gate data, the short gate bias adjustment assures the values should fall about the line of perfect agreement on average, but does not guarantee a necessarily adequate or consistent fit to the line. The short gate data show good consistency at all mass values. The amount of variability about the line is somewhat greater than that for the long gate data. This increased variability is consistent with the fact that the short gate measurements are generally more inaccurate, leading to the selection of the long gate results in all calibration cases.

The increased variability between simulated and actual PAN short gate measurements is more evident in Figure 8-4 which shows the relative percent error in each simulation measurement. The error bounds in the figure were calculated in the same manner as described above for the long gate data.

As can be seen from the graph, the relative error in the short gate measurement simulations can be an order of magnitude more than those seen for the long gate data. Note however that only part of these larger errors are attributable to the greater absolute variability observed in Figure 8-3. The relative calculations are also inflated because so many of the short gate measurements are very near zero. The error bounds shown in the graph indicate that the values are still within the range expected and so we still conclude that the short gate simulation process is performing appropriately.

\subsection{Comparison of Uncertainty Results}

While the previous sections compared simulated to actual PAN measurement results, this section compares simulated and actual PAN measurements to the true $\mathrm{Pu}$ quantities known to be in each drum. This provides a comparison of the PAN system uncertainty as estimated by the simulations vs. that obtained from actual drum assays.

Figure 8-5 compares plots of actual and simulated PAN measurements vs. the true Pu quantity in the calibration drums. (A full listing of the data is given in Appendix C, Table C-1.) The plots show that the simulated data mimic the actual PAN data quite closely in terms of their relationship to the true $\mathrm{Pu}$ 
quantities in the calibration drums. The simulation data compare favorably both in terms of the general trend in relation to the line of perfect agreement (which relates to bias) and the variability among repeat measurements at various $\mathrm{Pu}$ quantities (which relates to precision).

The bias and precision components of the simulated and real PAN data are more easily evaluated by considering the difference between the PAN results and the true Pu quantities. These data are shown in Figure 8-6. Absolute rather than relative errors are plotted in the figure because the absolute errors are more constant. As will be done for the full uncertainty analysis, bias and precision can be assessed by considering the fitted regression lines to the data in these plots. If there were no bias in the PAN system, all the difference data would average to zero. Hence, to the extent that the regression line deviates from a horizontal line through zero on the plot, bias is indicated. A regression intercept other than zero is indicative of a constant bias in the PAN system. A significant slope indicates a relative bias component. The extent of precision error is indicated by the variability of the absolute difference values about the regression line.

The regression equations in Figure 8-6 indicate that the simulated long and short gate PAN calibration data produce very nearly the same bias component estimates (absolute and relative bias) as the corresponding actual PAN calibration data. Neither the slope or intercept coefficients for the simulated data differ significantly (based on t-tests) from the corresponding coefficients for the actual data.

The spread of the data about the regression lines also appears similar in the simulated vs. actual PAN data plots, indicating comparable precision as well. A more precise comparison of the precision in each case can be obtained by considering the square root of the mean square error from each regression, often called the standard error of estimate. The mean square error is an estimate of the variance of the data about the regression line and hence its square root is a precision estimate. These values are given in Table 8-1. Note that the mean square error in a standard least squares regression model assumes a constant variance for all mass values when in actuality the PAN variance may increase with mass. This variance relationship is considered in detail in the waste drum simulation data, but for purposes of assessing the adequacy of the simulation process, the simplified equal variance model is sufficient.

Table 8-1. Precision estimates for simulated vs. actual PAN: mass calibration drum data.

\begin{tabular}{|l|c|c|}
\hline \multirow{2}{*}{} & Estimated precision (regression standard error of estimate) \\
\cline { 2 - 3 } & Long gate mass & Short gate mass \\
\hline Actual PAN data & 2.9 & 3.9 \\
\hline Simulated PAN data & 3.5 & 4.2 \\
\hline
\end{tabular}

Table 8-1 shows that the standard errors of estimate for the simulated data are somewhat larger than the corresponding values for the actual data. This is acceptable from an uncertainty estimation standpoint as it produces conservative values regarding the accuracy of PAN measurements. The calibration simulation data also indicate that the agreement of the simulated values with the actual measured values is much better than the agreement of the actual measured values with the corresponding true Pu quantity in the drum. Thus any deviations of the simulated values from the actual measured values are likely to be small relative to the variability of the PAN system itself to changes in factors such as drum configuration.

Note that all the values reported above measure the uncertainty for the PAN system only for the calibration drum configurations, and should not be interpreted as indicative of the overall performance of the PAN system on glass waste drums. Overall performance should be based on the results for the simulated waste drum sample reported in the next section. 
It should also be noted that in the actual PAN calculations, and therefore in the simulation of the PAN calculations, the isotopic correction factor was taken to be 1.0. This implies that the mass fraction of Pu240 is 0.0582 (i.e., this is the nominal value assumed in the PAN calculations for weapons grade plutonium). The actual Pu-240 mass fraction for the NADs is .0528 . Hence for calibration runs involving NAD sources, this value was used in simulating the PAN response in terms of singles and coincidence counts. Similarly, the response for calibration runs using ZPPR plate sources were simulated using a mass fraction value of .0451 (based on an average of $10 \mathrm{ZPPR}$ plates). If isotopic correction factors of $0.0582 / 0.0528=1.10$ for calibration runs using NADs and $.0582 / .0451=1.29$ for those using ZPPR plates had been used in the PAN analysis, the difference between the actual and PAN reported mass values (actual or simulated) might be much closer. However, it is still appropriate to use an isotopic correction factor of 1.0 in the PAN calculations because that is the assumed (default) value in the actual operation of the PAN system, unless gamma spectrometric data indicates conclusively to the contrary. 
Figure 8-1. Comparison of simulated vs. actual PAN system long gate mass values for glass calibration drums

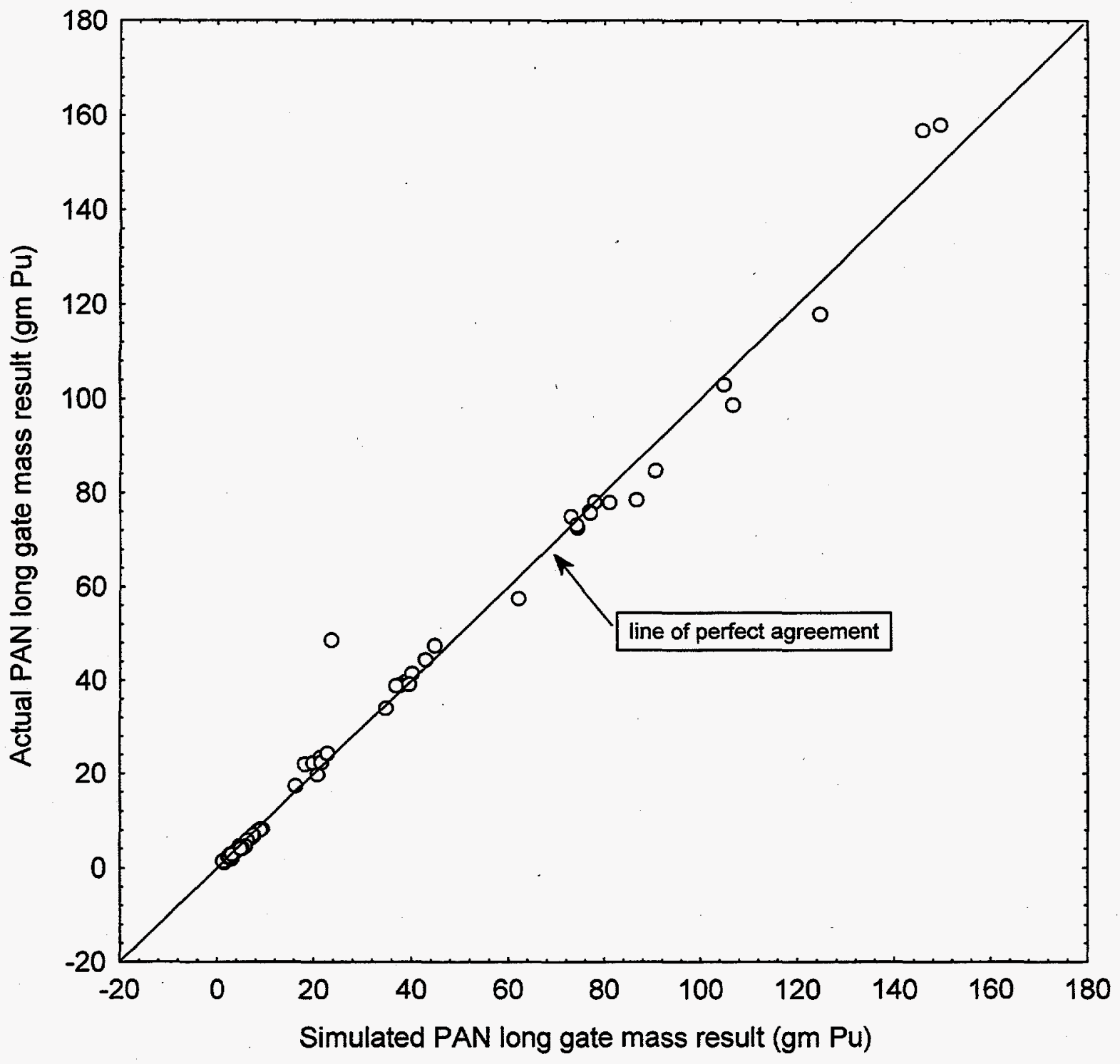


Figure 8-2. Relative error in simulated vs. actual PAN system long gate mass values for glass calibration drums

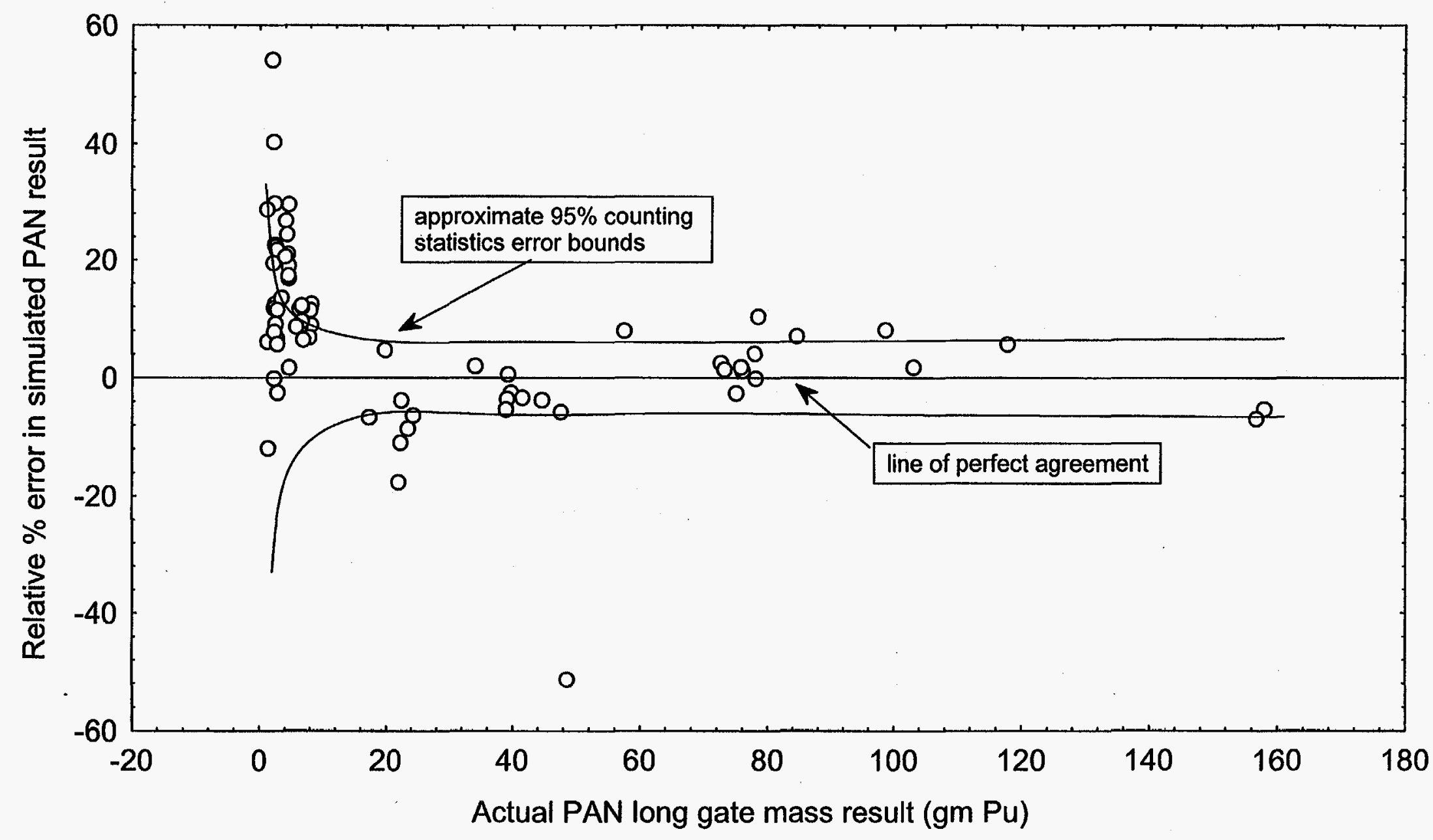


Figure 8-3. Comparison of simulated vs. actual PAN system short gate mass values for glass calibration drums

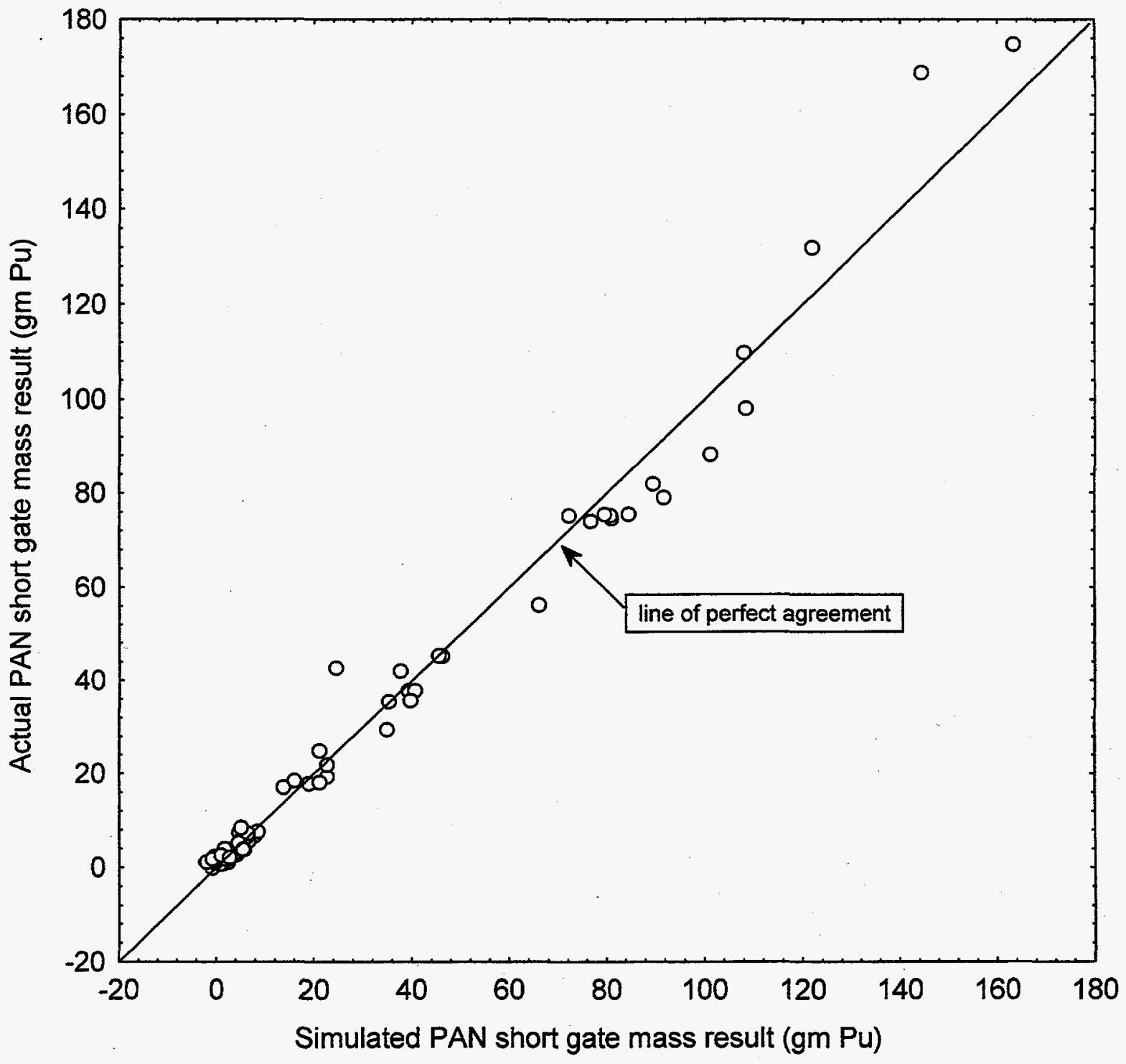




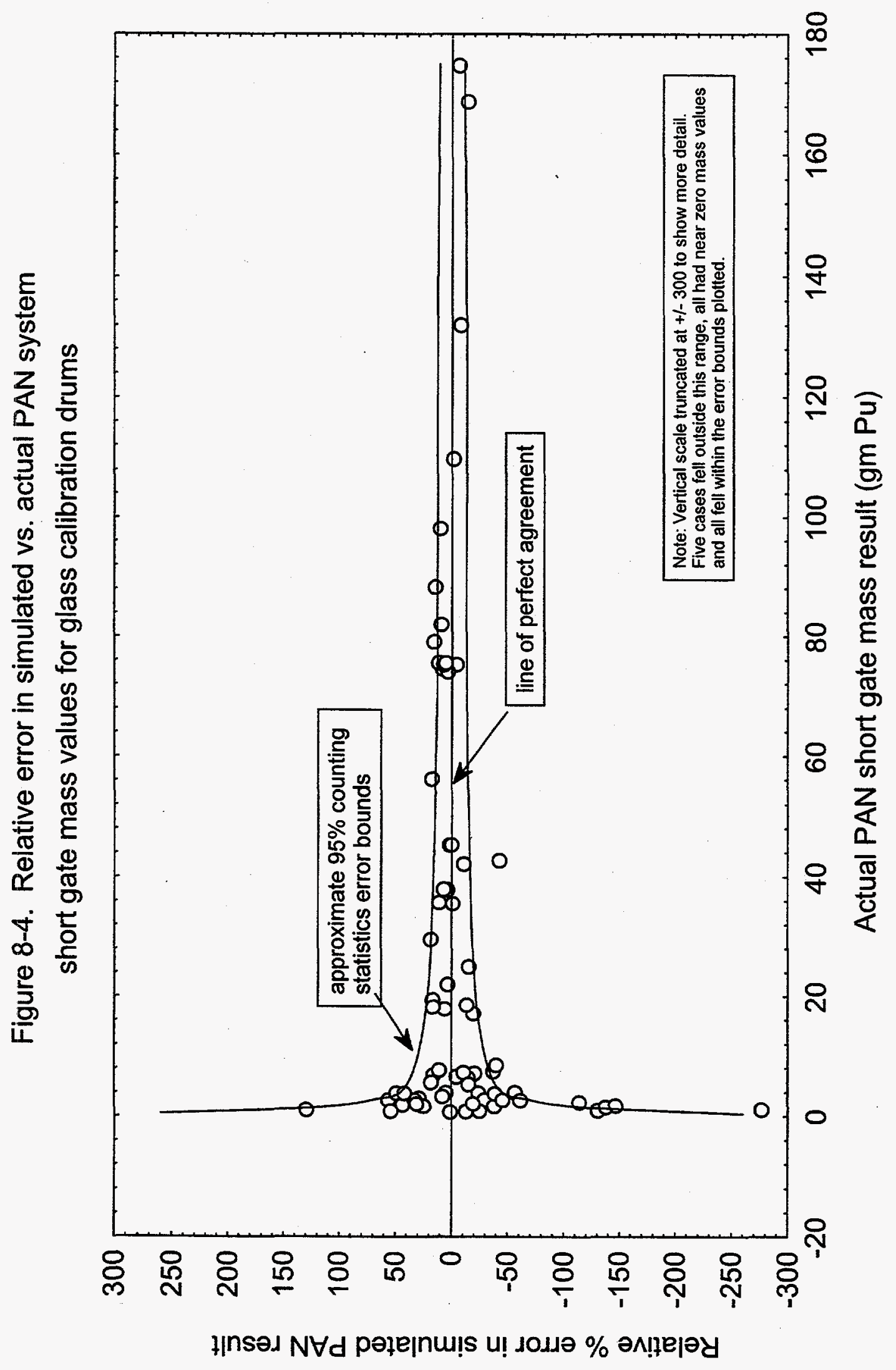



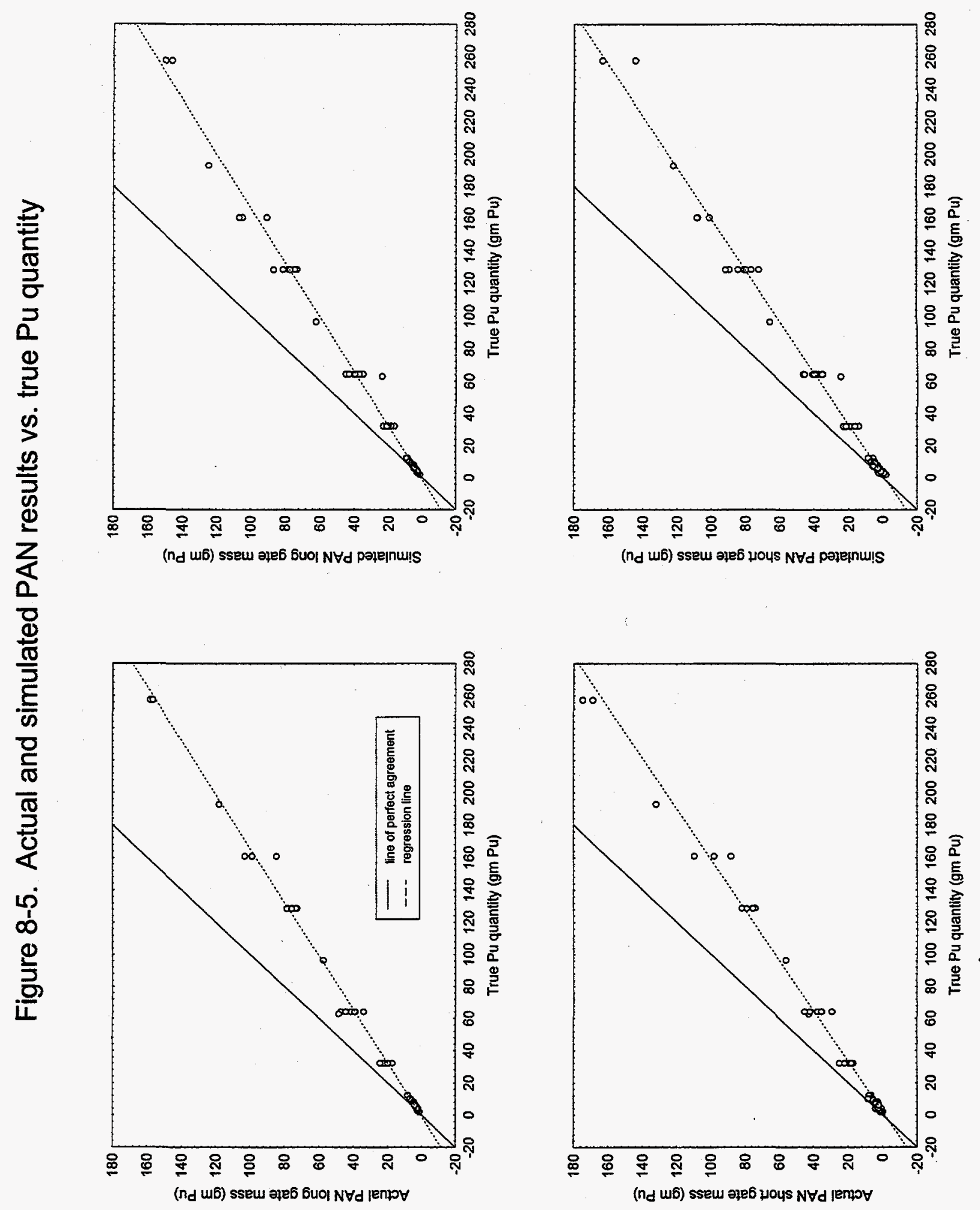
Figure 8-6. Comparison of deviations from true Pu quantities
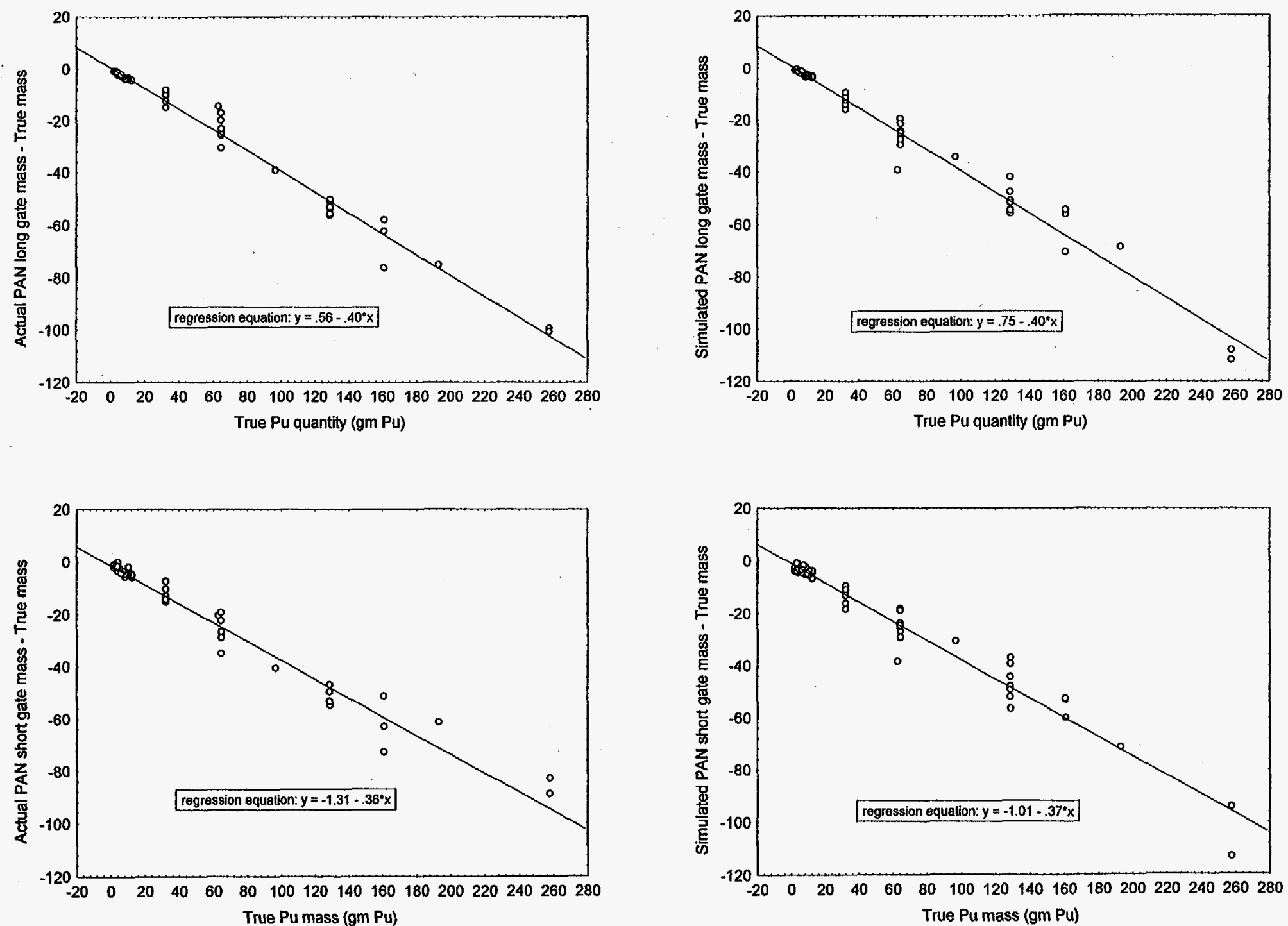


\section{UNCERTAINTY ANALYSIS USING THE SIMULATED WASTE DRUM SAMPLE RESULTS}

The PAN analysis passive measurement results for the 100 simulated glass waste drum configurations are given in Appendix C, Table C-2. For the passive mode, the PAN analysis selects either the short gate or long gate coincidence Pu mass as the final reported mass depending on the calculated errors. The value with the smallest relative error is chosen. For the 100 simulated glass waste drums, the PAN analysis selection criteria chose the long gate mass as the final estimate of Pu mass in 90 cases and the short gate mass in 10 cases. The uncertainty in each result is obtained by comparison to the Pu quantity specified for each drum. (For brevity, the Pu quantity specified for each simulated drum will generally be referred to in this section as the "actual" or "true" quantity, and the simulated PAN system measurement result as simply the "PAN Pu" quantity. Also, the PAN Pu quantities have been adjusted for the bias discussed in Section 8.2.)

The true vs. PAN measured Pu quantities are plotted in Figure 9-1. The line of perfect agreement specified in the plot indicates where the data would fall if there were no measurement bias and/or precision error. The second line in the plot is a regression line fit to the data using the method described below. That the regression line falls below the line of perfect agreement indicates a negative bias exists in the PAN system results. The degree of the scatter in the points about the regression line is an indicator of the degree of precision in the measurements.

Bias and precision in the results can be quantified by considering the following basic mathematical model for the data in Figure 9-1:

$y_{i}=\alpha+\beta\left(x_{i}\right)+\varepsilon_{i}$

where $y_{i}$ is the PAN system Pu measurement for drum $i$ and $x_{i}$ is the true Pu quantity in the drum. The parameters $\alpha$ and $\beta$ relate to the bias in the measurement process. If $\alpha \neq 0$ and $\beta=1$, then there is a constant bias in the measurement system. If $\alpha=0$ and $\beta \neq 1$, then there is constant relative bias in the system. It is also possible to have both constant and relative bias terms at the same time. The term $\varepsilon_{\mathrm{i}}$ in the model is the random error component of the measurement. Precision of the measurement system is evaluated by considering the variance (or equivalently the standard deviation) of the $\varepsilon_{\mathrm{i}}$ values.

Using this basic measurement model for the PAN system, bias and precision can be evaluated by applying appropriate statistical analysis to the data from the simulations. These calculations are described in detail below.

\subsection{Bias}

Simply defined, bias is the expected difference between the measured and actual Pu quantities. Thus it can be assessed more directly by subtracting the true $\mathrm{Pu}$ quantity $\mathrm{x}_{\mathrm{i}}$ from both sides of Equation 9.1 to give:

$$
\begin{aligned}
\mathrm{y}_{\mathrm{i}}-\mathrm{x}_{\mathrm{i}} & =\alpha+\beta\left(\mathrm{x}_{\mathrm{i}}\right)-\mathrm{x}_{\mathrm{i}}+\varepsilon_{\mathrm{i}} \\
& =\alpha+\beta^{*}\left(\mathrm{x}_{\mathrm{i}}\right)+\varepsilon_{\mathrm{i}}
\end{aligned}
$$


where $\beta^{*}=\beta-1$. The meanings of $\alpha$ and $\varepsilon_{\mathrm{i}}$ remain the same as before. $\beta^{*}$ still relates to relative bias, but now no relative bias results in a value of 0 for $\beta^{*}$. Based on this equation then, the bias in a PAN measurement of $\mathrm{x}$ grams $\mathrm{Pu}$ is simply $\alpha+\beta^{*}(\mathrm{x})$, the expected value of Equation 9.2. Since the parameters $\alpha$ and $\beta^{*}$ are not known they must be estimated using regression techniques applied to the simulated waste drum data.

The data for the revised model in Equation 9.2 are plotted in Figure 9-2. If there were no bias in the measurements, the data in Figure 9-2 would be centered around the line of perfect agreement. (Variability about the line represents the random error or precision of the measurements.) That the data points tend to fall below this line indicates once again a negative bias in the PAN measurement system.

A regression analysis was performed to estimate the parameters $\alpha$ and $\beta^{*}$ in Equation 9.2. Since the variability of the data increases with increasing true $\mathrm{Pu}$ quantity, a weighted least squares analysis was performed (Neter and Wasserman, 1974). Ideally, weights should be $1 / \sigma_{x}{ }^{2}$ where $\sigma_{x}{ }^{2}$ is the variance of $\varepsilon$ for a given value of $x$. Note that these variances are also the desired precision estimates for the PAN system (as defined in Equation 9.1). Since these values are not known, they must be estimated. The method of estimation is described in Section 9.2.

\subsubsection{Weighted Least Squares Results}

Using the weights defined by the precision estimates, a weighted least squares analysis of the model in Equation 9.2 was performed. The first test in the analysis was to consider the presence of a quadratic effect in the bias trend as a means of assessing the validity of the linear model stated in Equation 9.2. The test for a quadratic effect had a p-value of .90 . Hence there is no indication of significant departure from linearity in the bias trend (i.e., Equation 9.2 is an appropriate model for the data).

Fitting a strictly linear equation to the data produced an estimate of $\alpha$ that was not significantly different from zero $(p=.43)$, hence that parameter was dropped from the model. In the reduced model with $\alpha$ set to zero, the effect of $\beta^{*}$ was highly significant $(p<.001)$. Substituting 0 for $\alpha$ and the estimated value of -0.24 for $\beta^{*}$ into Equation 9.2 and taking expectations of both sides gives the following simple model for bias in the PAN system.

bias $=-0.24$ (true Pu mass).

In other words, the PAN system produces measured values that are $24 \%$ lower than the true $\mathrm{Pu}$ quantities. The standard error of the estimated value for $\beta^{*}$ is .028 , so an approximate $95 \%$ confidence interval on the $-24 \%$ relative error value is $(-18 \%,-30 \%)$. The inaccuracy in the bias estimate is due to the variability in the data.

\subsection{Precision}

As indicated in Figure 9-2, variability of the PAN response increases with increasing Pu mass. Hence precision was estimated by fitting an appropriate smooth function to standard deviations calculated for each of the 10 sets of 10 replicate Pu mass measurements $(1,3,5,10,20,30,40,60,80$, and 100g). These data are plotted in Figure 9-3. 
A regression analysis of these data indicated that standard deviation is a nonlinear function of plutonium mass. A quadratic equation was used to estimate the relationship:

standard deviation $=2.0+.0034$ (true Pu mass) $)^{2}$.

The linear term in the quadratic regression was clearly not significant $(p=.65)$ so was eliminated from the model. The intercept term in the equation did not quite meet the .05 level of significance $(p=.054)$, but was left in because of the known fact that a certain amount of variability will occur even in repeat measurements on an empty drum. (Excluding an intercept term would produce zero variability at zero grams Pu.) In addition to its use as a representation of precision for glass waste drum measurements in the PAN system, Equation 9.4 was also used to derive the weights used in the weighted least squares regression for bias estimation in the previous section.

\subsubsection{Comparison to Counting Statistics Error}

It is of interest to compare the precision estimates obtained from Equation 9.4 to the standard deviations reported automatically by the PAN system. The PAN system estimates of standard deviations include variability due to counting statistics only. Since standard deviations obtained from Equation 9.4 also include matrix effects etc., they should in general be larger than the PAN reported values. The PAN reported standard deviations are plotted in Figure 9-4. A weighted least squares fit to the PAN reported data is also plotted and compared to the formula in Equation 9.4. The analysis showed that a linear fit to the data was appropriate. (The p-value for a quadratic term was .99). The plot shows the values from Equation 9.4 are actually less than the PAN fitted value line over the intermediate range of Pu quantities. This anomaly is to a large extent due to the amount of variability observed in the PAN data and the fact that the PAN reported values are positively skewed. Other methods of fitting a line to the PAN data produced different results. For example, a fit to the median PAN reported standard deviation values at each Pu quantity produced a line which always falls below the plot for Equation 9.4, as indicated in Figure 9-5. Additional work is planned to determine the best method of quantitatively comparing the two sets of results.

It should be noted that one major matrix-related parameter contributing to the precision error is the $(\alpha, n)$ singles rate. The variability in this rate also affects the counting statistics error reported by the PAN system. So in that regard the reported counting statistics error actually already contains some additional variability (i.e., that due to $(\alpha, n)$ interference).

\subsection{Matrix Parameter Effects on Uncertainty}

The analysis of data related to Equations 9.1 and 9.2 established a relationship between the error in a measurement and the Pu quantity being measured. It is also of interest to consider whether any of the matrix parameters have consistent effects on the measurement error $y_{i}-x_{i}$. This was accomplished by performing a statistical analysis using key matrix parameters determining the simulated waste drum configurations. These include categorical parameters (number of fiber packs, content code and whether or not the plutonium occurs in fines or chunks) and the continuous modeled parameters (absorber index, average fill height, mass of the drum contents, the shielded and system $(\alpha, n)$ singles rates, Pu-240 mass fraction, and the mass fractions of the other elements making up the matrices in the drums).

Note that effects of the various parameters, especially the elemental mass fractions are not independent (because of the requirement that the mass fractions sum to unity, when one percentage goes up, the remaining ones must go down). To specify the effects in detail, a complicated mixture model analysis of 
variance would be needed. But for the purpose of this analysis (i.e., to get a general idea of which parameters have noticeable effects), examination of the individual effects using t-tests, correlations, and other methods, interpreting them in light of the known dependencies, is sufficient.

\subsubsection{Parameter Effects on Bias}

Because Equations 9.2 and 9.3 specifies a known effect of Pu quantity on the error, the quantities $y_{i}-x_{i}$ were adjusted prior to the analysis of parameter effects using the formula:

adjusted value $=\mathrm{y}_{\mathrm{i}}-\mathrm{x}_{\mathrm{i}}+.24\left(\mathrm{x}_{\mathrm{i}}\right)$.

In regression terms the adjusted values are the residual values remaining from the regression analysis of the model in Equation 9.2. The residuals are essentially measurement errors adjusted for the bias due to Pu quantity. Hence the results below refer to the effects of other matrix parameters after the previously identified effect of Pu quantity has been taken into account. The result is that any significant effects identified will indicate bias effects of the matrix parameters that are in addition to the bias due to Pu quantity.

Effects of the continuous scale parameters were tested using Pearson's $\mathbf{r}$ correlation coefficient. Parameters with significant $(\mathrm{p}<.05)$ or nearly significant $(.05<\mathrm{p}<.10)$ are listed in Table $9-1$. The $\mathrm{p}$ values given are for the test of the null hypothesis that the correlation is zero. Thus, low p-values (e.g., $p$ $<.05)$ indicate statistically significant correlations.

Table 9-1. Significant or near significant correlations of matrix parameters with adjusted deviation data (indicating effects on bias).

\begin{tabular}{|l|c|c|}
\hline \multicolumn{1}{|c|}{ Matrix parameter } & Correlation & p-value \\
\hline Absorber index & .36 & .00 \\
\hline Mass fraction of carbon & -.21 & .00 \\
\hline System $(\alpha, n)$ singles rate & .17 & .09 \\
\hline
\end{tabular}

All three of the correlations in Table 9-1 are due to two or three outlier values, without which the correlations are not statistically significant. (The correlation with system $(\alpha, n)$ singles rate actually becomes negative without the two highest valued cases.) Thus, substantive conclusions can not be drawn from these data.

The categorical parameters were tested using analysis of variance (i.e., ANOVA) techniques. Both standard ANOVA techniques (which tests for differences between means) and nonparametric ANOVA techniques (which tests for differences between medians) were utilized. In terms of significance of effects the results were consistent between the two methods. Significant effects were found for the effects of $\mathrm{Pu}$ in chunks vs. fines, and content code. The mean adjusted difference for cases with $\mathrm{Pu}$ in fines was $-4.5 \mathrm{~g}$ while for cases with $\mathrm{Pu}$ in chunks it was $5.1 \mathrm{~g}$. The difference in medians was $-1.4 \mathrm{~g}$ for fines vs. $1.0 \mathrm{~g}$ for chunks.

For content codes, the mean adjusted difference values were -1.6 for code $440,23.1$ for code 441 , and -1.0 for code 442 . The corresponding median values were $0.7,5.8$, and -0.5 respectively. Thus the biggest difference is between the code 441 drums and the others. There are only six code 441 drums in the simulated data. The significant difference appears due to only two of the six drums. These two drums were also two of the outlier cases identified earlier in the correlation analysis. Examination of the characteristics of these two drums did not reveal any single factor differentiating them from other drums. 


\subsubsection{Parameter Effects on Precision}

The effects of the various parameters on precision were examined by considering correlations with the absolute values of the adjusted differences calculated in Equation 9.5. The absolute value of a difference can be interpreted essentially as a standard deviation estimate based on a single observation. Thus, parameters with large effects on precision errors should show significant correlations with the expected magnitude (i.e., absolute value) of the differences between measured and observed values. Because the standard deviation of differences is known to vary by Pu quantity as described earlier, the absolute deviation values were normalized prior to analysis by dividing by the standard deviation for the relevant $\mathrm{Pu}$ mass as calculated in Equation 9.4. Thus the correlations represent effects of the parameters on precision in excess of that attributable to Pu mass. To reduce the skewness of the data, the analysis was performed on the logarithms of the normalized deviation data. Correlations with elemental mass fractions were only considered when there were at least 10 drums containing a particular element.

None of the categorical variables showed significant effects. Significant $(p<.05)$ correlations of matrix parameters with the adjusted and normalized absolute deviations are given in Table 9-2.

Table 9-2. Significant correlations of matrix parameters with the logarithms of the adjusted and normalized absolute deviations (indicating effects on precision).

\begin{tabular}{|l|c|c|}
\hline \multicolumn{1}{|c|}{ Matrix parameter } & Correlation & p-value \\
\hline System $(\alpha, n)$ singles rate & .22 & .00 \\
\hline Shielded $(\alpha, n)$ singles rate & .22 & .00 \\
\hline
\end{tabular}

Only the $(\alpha, n)$ singles rates showed significant correlations with precision error. The values for shielded and system counts are equal due to their high correlation with each other. The $(\alpha, n)$ interference effect has been seen in uncertainty analysis of other waste types (i.e., graphite and combustibles). The $(\alpha, n)$ interference is an inherent problem with coincidence systems as used in the PAN passive mode. In order to get sufficient sensitivity with the He-3 detectors mounted inside polyethylene moderators it is necessary to operate the coincidence units with relatively wide gate widths (i.e., $35 \mu$ s for short-gate coincidence and $250 \mu \mathrm{s}$ for long-gate coincidence). Normal coincidence systems used in nuclear physics applications operate with gate widths which are much smaller (e.g., $5 \mu$ s or less). Therefore, the probability for chance coincidence counts are much higher with the wider gate widths. Obtaining the real coincidence rate with the PAN gate widths becomes a problem of taking the difference between two large statistically varying numbers and therefore the difference will be highly uncertain. These factors lead to the increased variability in results. 


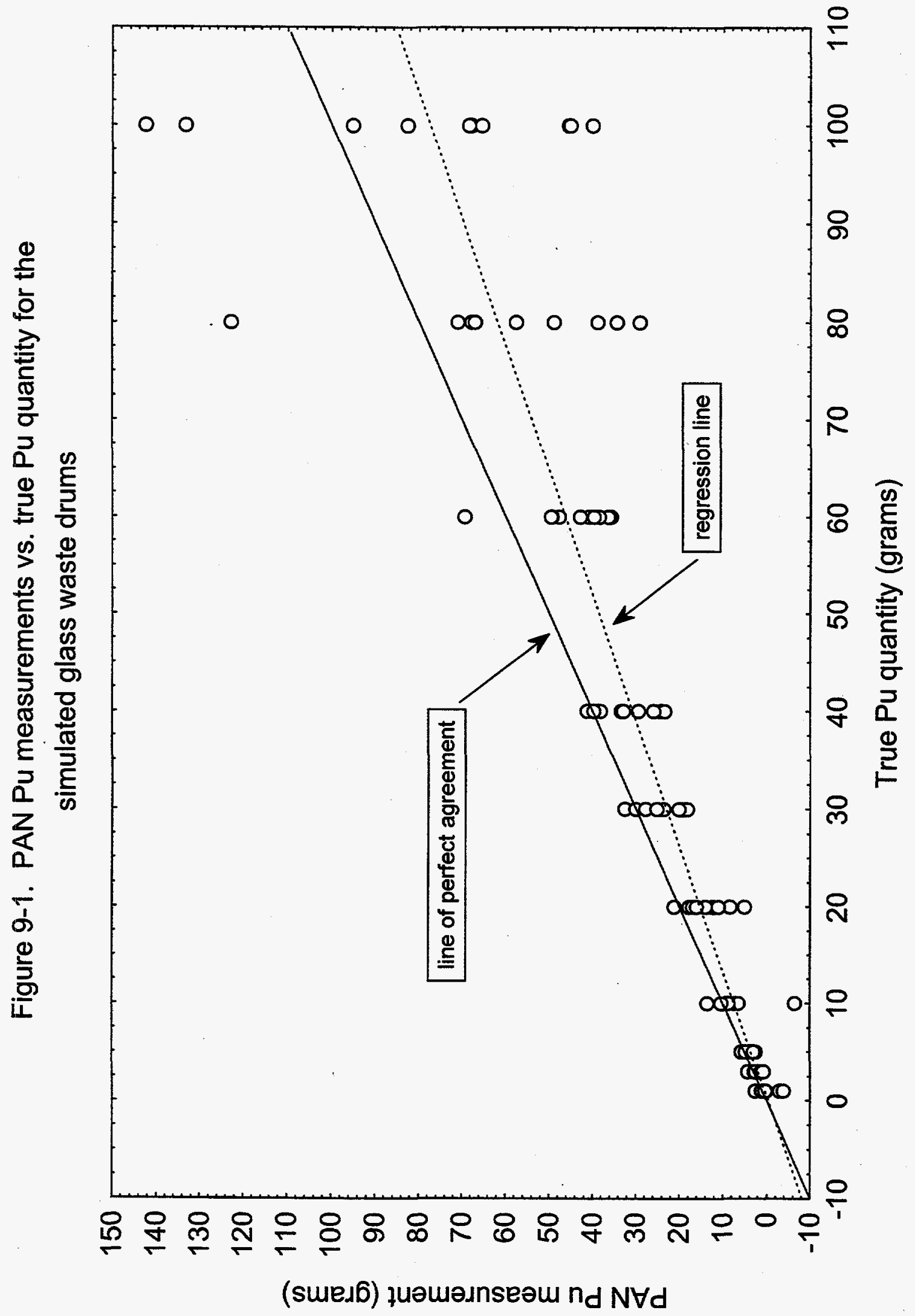




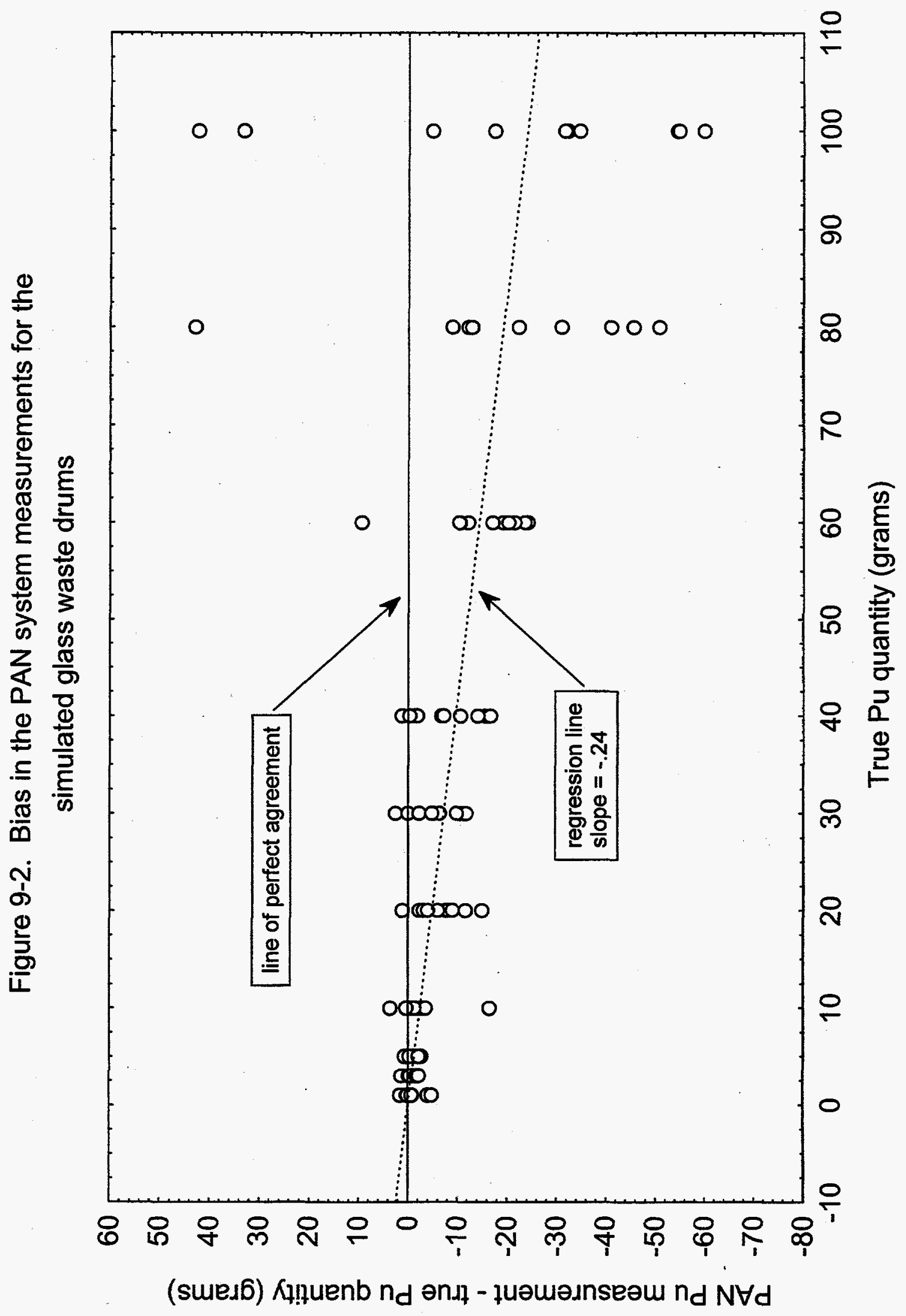


Figure 9-3. Standard deviations of replicate data for the simulated glass waste drums

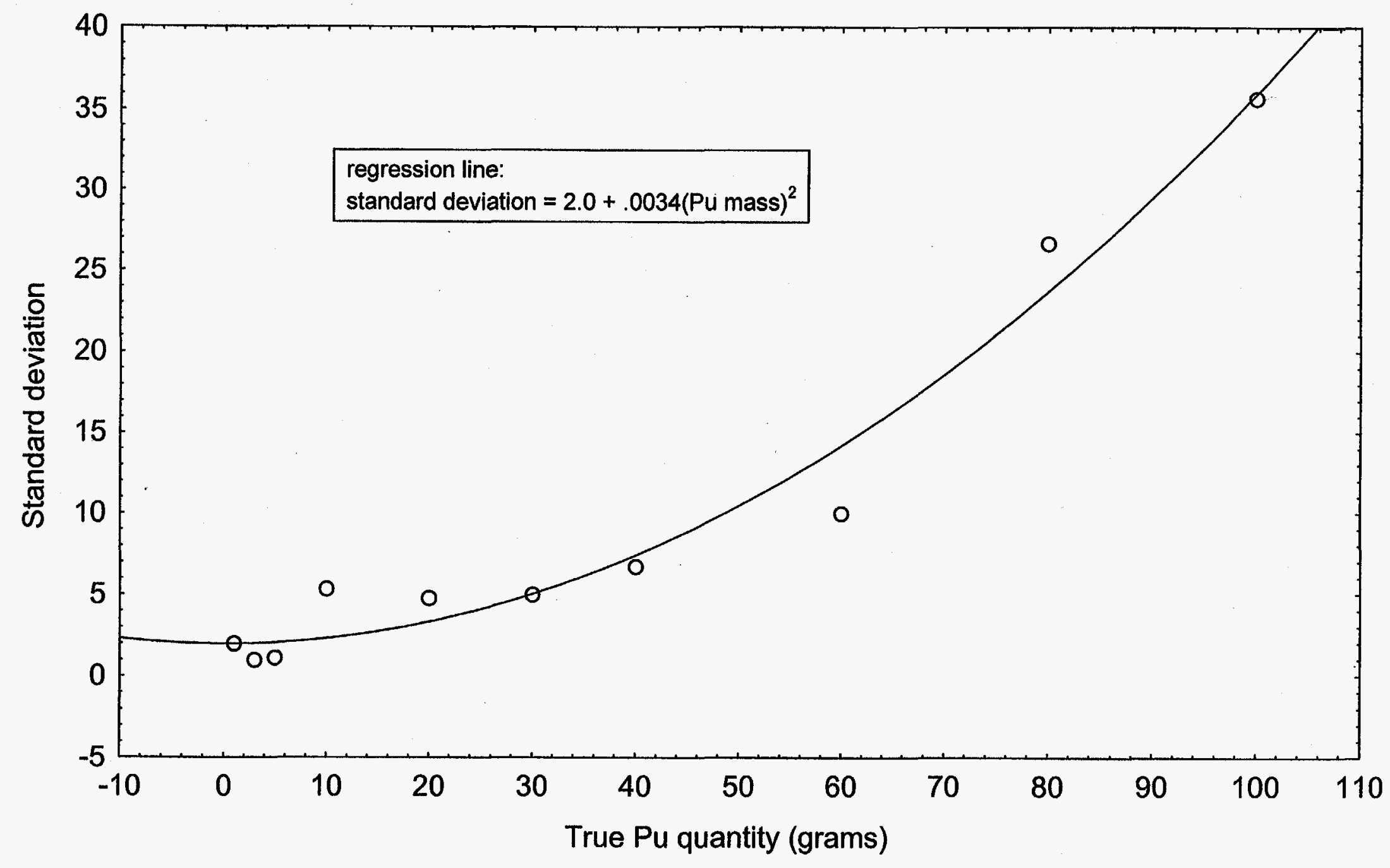


Figure 9-4. Comparison of Equation 9.4 results and regression fit to individual PAN reported standard deviations for the simulated glass waste drums

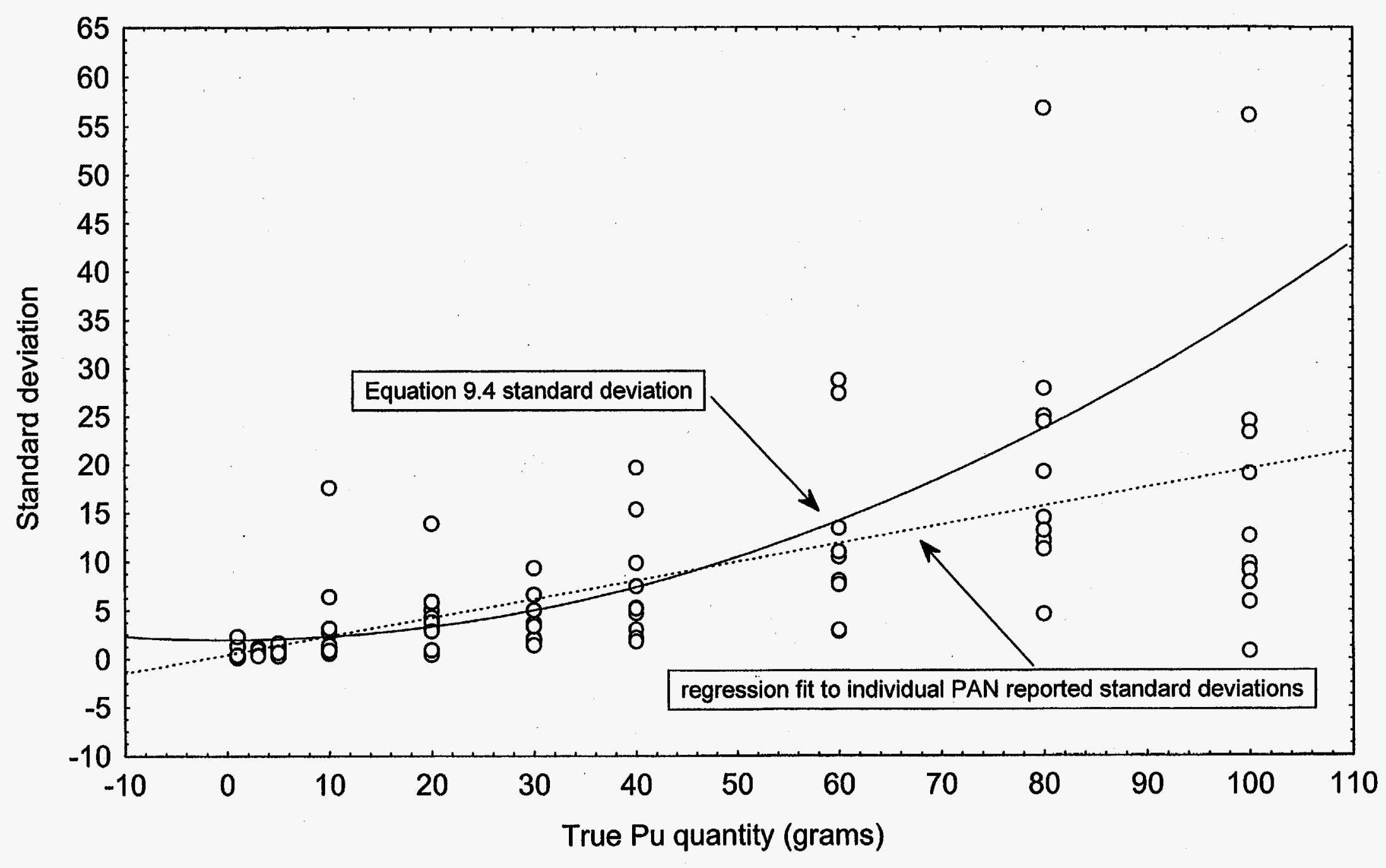


Figure 9-5. Comparison of Equation 9.4 results and regression fit to median

PAN reported standard deviations for the simulated glass waste drums

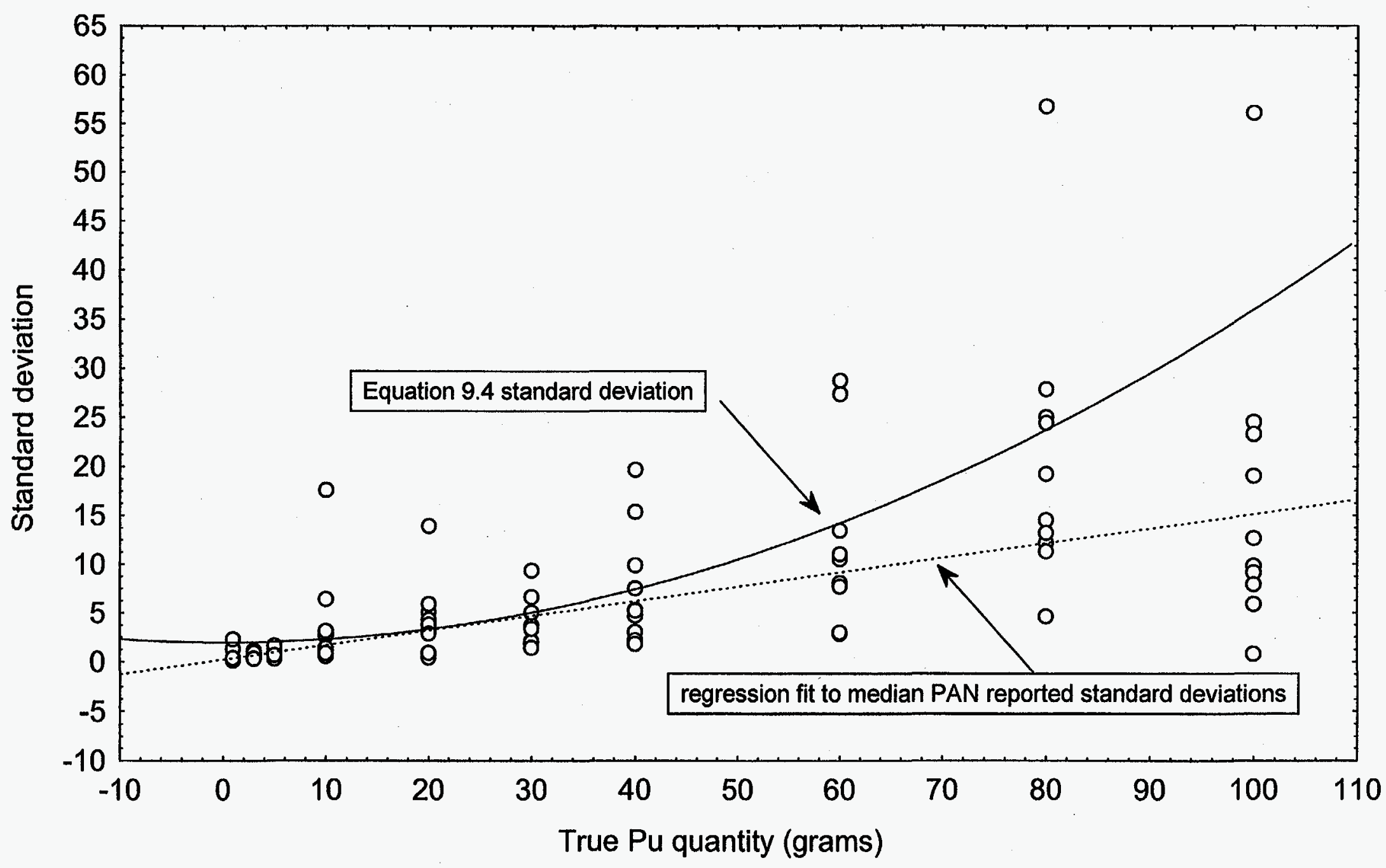




\section{SUMMARY AND DISCUSSION OF RESULTS}

This report presented a methodology for determining the total uncertainty of the SWEPP PAN assay system and applied this methodology to the measurement of glass waste (content codes 440,441 , and 442). In order to perform this evaluation, a complete assessment of the physical and radiological characteristics of glass waste drums was completed by reviewing real time radiography tapes, data from past measurements using the PAN assay system, and shipping records from Rocky Flats Plant. Based on the data analysis, a multivariate statistical model was developed which describes the configuration of parameters which could alter the response of the assay system. The primary parameters in this model are: mass of weapons grade Pu, mass fraction of Pu-240, fill height of the drum, matrix density, elemental composition of the waste, absorber index, background singles counts, background coincidence counts, and $\mathrm{Pu}$ contained in fines vs. chunks. Using the statistical model, a set of 100 computer simulated glass waste drums were prepared for input into a computer simulation of the assay system response and analysis routines.

The computer simulation of the PAN assay system response involved a neutron transport calculation using MCNP and a follow-up program which uses the MCNP results along with other parameters in the statistical model to determine the singles and coincidence count rate responses. These count rate data were then analyzed to yield Pu mass using exactly the same algorithms as contained in the PAN analysis program. The accuracy of the combined simulation routine was verified using data taken from measurements involving two glass waste calibration drums and known Pu sources. From these comparisons small adjustment factors were derived to make the simulated $\mathrm{Pu}$ mass quantities agree on average with the Pu mass determined from the PAN measurement. These adjustment factors were included in the computer simulation routine in all analyses involving the simulated waste drum cases so

that there was no bias in the simulation results relative to the expected PAN results. Comparison of the simulated vs. actual PAN measured results indicate that the random variability of the simulated and PAN results are quite comparable.

Analysis of the results for the simulated waste drums indicates that, on average, the reported PAN Pu mass for glass waste drums is biased low by $24 \%$ and the standard deviation varies quadratically from about $2 \mathrm{~g}$ at a nominal $1 \mathrm{~g}$ of weapons grade $\mathrm{Pu}$ to $36 \mathrm{~g}$ at $100 \mathrm{~g}$. Specifically, the formula for bias is:

bias $=-.24$ (true Pu mass),

while the formula for precision (in terms of standard deviation) is:

standard deviation $=2.0+.0034(\text { true } \mathrm{Pu} \text { mass })^{2}$.

\subsection{Propagation of Total Uncertainty for Measured Mass Values}

In the previous sections, bias and precision estimates are reported relative to the true Pu mass (i.e., the mass specified in the simulation). It is also helpful to convert these estimates so that they are relative to the measured Pu mass. Then, given the measured mass, we can calculate a confidence interval (based on the total uncertainty) within which the true mass is expected to fall. These calculations were obtained as follows.

Based on Equation 10.1, the expected bias can be restated as

(measured Pu mass) - (true Pu mass) $=-.24$ (true Pu mass). 
Solving for the true Pu mass gives

true Pu mass $=1.32$ (measured Pu mass).

The applicable precision for a particular measured Pu mass is the standard deviation specified in Equation 10.2 applied to the bias corrected mass value:

standard deviation $=2.0+.0034(1.32(\text { measured } \mathrm{Pu} \text { mass }))^{2}$

$$
=2.0+.0059 \text { (measured Pu mass })^{2} \text {. }
$$

To get approximate $95 \%$ uncertainty bounds on a measured mass value, consider propagation of error for Equation 10.4, acknowledging that both the measured $\mathrm{Pu}$ mass and the bias parameter have associated precision error. Treating the bias as a parameter, Equation 10.4 can be restated as

true Pu mass $=(1+b)^{-1}($ measured Pu mass $)$

where $b=-.24$ with standard error $s_{b}=.028$ as discussed in Section 9 .

Let $s_{\text {true }}$ be the standard deviation obtained from Equation 10.5. Taking partial derivatives of Equation 10.6 with respect to $\mathrm{b}$ and the measured $\mathrm{Pu}$ mass, and applying standard propagation of error formulas yields the following equation for the standard deviation of the adjusted measured Pu quantity:

$s_{\mathrm{adjPu}}=\left[\left((1+b)^{-1}\right)^{2}\left(\mathrm{~s}_{\mathrm{true}}\right)^{2}+\left((\text { measured Pu mass })(1+b)^{-2}\right)^{2}\left(s_{b}\right)^{2}\right]^{1 / 2}$

Substituting the previously estimated values for $b, s_{t r u e}$, and $s_{b}$ gives

$$
\begin{aligned}
\mathrm{S}_{\mathrm{adjPu}}= & {\left[( ( 1 - . 2 4 ) ^ { - 1 } ) ^ { 2 } \left(2.0+.0034\left(1.32(\text { measured Pu mass })^{2}\right)^{2}\right.\right.} \\
& \left.+\left((\text { measured Pu mass })(1-.24)^{-2}\right)^{2}(.028)^{2}\right]^{1 / 2} \\
= & {\left.\left.[6.7+.042 \text { (measured Pu mass })^{2}+.000060 \text { (measured Pu mass) }\right)^{4}\right]^{1 / 2} }
\end{aligned}
$$

An approximate $95 \%$ confidence interval for the true mass is:

1.32 (measured Pu mass) $\pm 2 \mathrm{~s}_{\mathrm{adjPu}}$.

Using the results of this evaluation we can make comparisons with performance criteria as established in Table 9-1 of the TRU Waste Characterization Quality Assurance Program Plan (DOE, 1995). The parameter of interest in this table is "Total Uncertainty" which is what has been evaluated in this report. The performance criteria are given in terms of total alpha activity, but we can make limited comparisons based on Pu mass. Shown in Figure 10-1 is the confidence interval for the true Pu mass plotted against the measured Pu mass. Also shown on this figure are the upper and lower limits as contained in Table 91 of the QAPP. This figure shows that the performance criteria (based on Pu mass only) can be met for measured PAN measurements in the range from approximately $8 \mathrm{~g}$ to $50 \mathrm{~g} \mathrm{Pu}$ for drums containing glass waste. 
New criteria have recently been proposed for Table 9-1 of the QAPP. These criteria only impose restrictions on the bias component of the total uncertainty. These criteria vary by activity level. The most restrictive of these criteria indicate that the smallest allowable lower limit on the $95 \%$ confidence interval for relative bias is $-33 \%$ and the maximum allowable upper limit is $150 \%$. As stated above, the expected bias for glass is $-24 \%$ with a standard error of $2.8 \%$. An approximate $95 \%$ confidence interval for the true bias is then $(-30 \%,-18 \%)$. This interval applies to all mass levels. Hence, based on uncertainty for the PAN measured Pu mass, the new criteria for Table 9-1 can be met for glass waste. (The final uncertainty must also include the uncertainty in the isotopic ratios used to convert the PAN computed $\mathrm{Pu}$ mass to curie content. The additional uncertainty may mean that the bounds are exceeded for some measurements, unless a bias correction is applied.)

In order to be critically safe, there is a safety limit requiring that the measured Pu mass plus $2 s_{\varepsilon}$ (i.e., the upper bound in Figure 10-1) must not exceed $200 \mathrm{~g}$. Based on the equations used to derive Figure 10-1 the measured mass must be below $77 \mathrm{~g}$ in order to meet this criterion for drums containing glass waste. (Again the uncertainty will be somewhat greater when isotopic ratios are considered.) 
Figure 10-1. Uncertainty bounds for glass drum measurements

(bias adjusted and accounting for uncertainty in bias estimate)

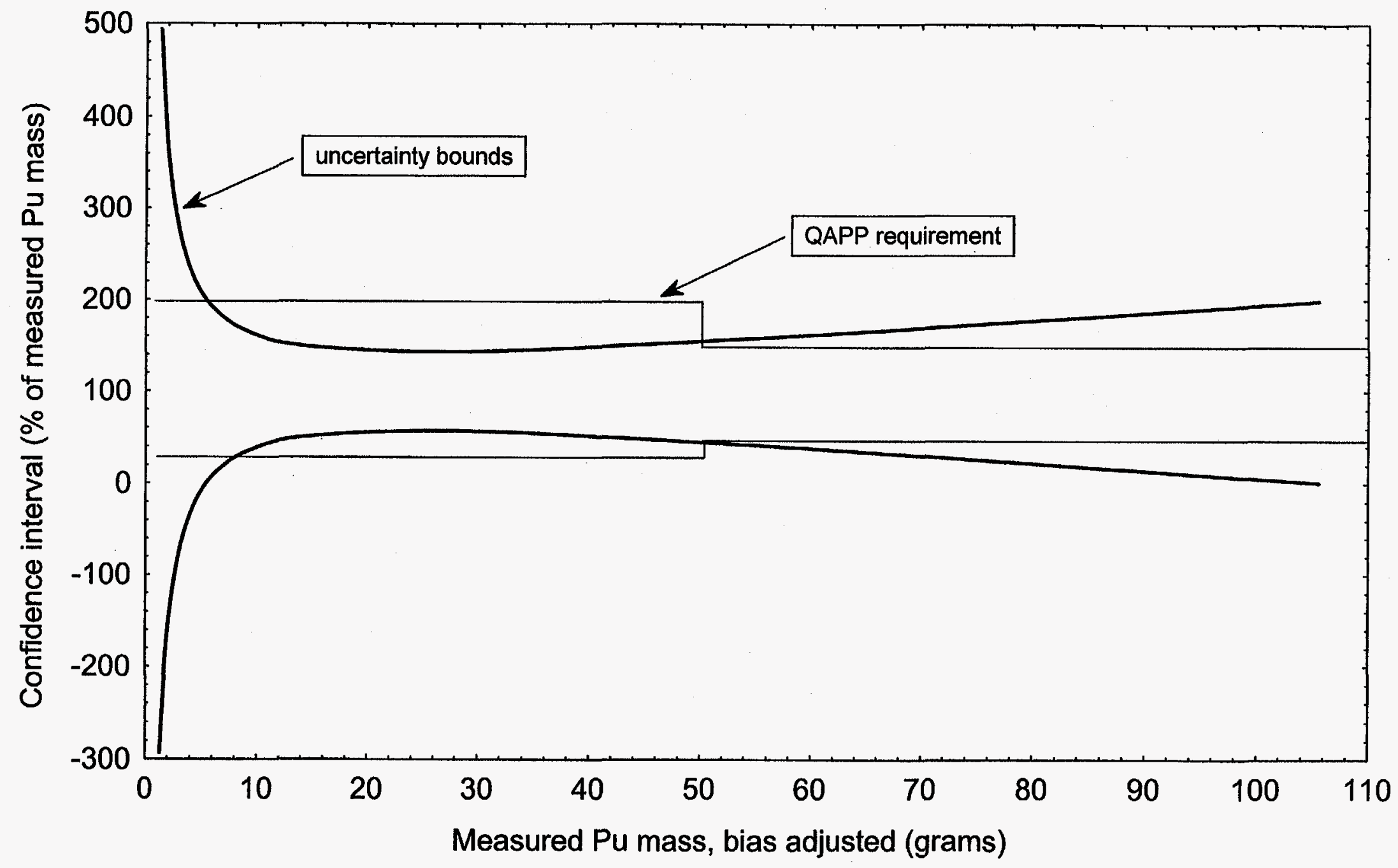




\section{REFERENCES}

Becker, G. K. (1992). "Content Code 442 Calibration Drum Design and Data," EDF-RWMC-529, Idaho National Engineering Laboratory, Idaho Falls, ID.

Becker, G. K. (1993a). "Drum Neutron Counter Chamber and Detector Configuration," Idaho National Engineering Laboratory Engineering Design File Document EDF-RWMC-606.

Becker, G. K. (1993b). “Content Code 440 Calibration Drum Design and Data," EDF-RWMC-560, Idaho National Engineering Laboratory, Idaho Falls ID.

Briesmeiser, J. F. (1986). "MCNP - A General Purpose Monte Carlo Code for Neutron and Photon Transport, Version 3A," Los Alamos National Laboratory manual LA-7396-M Rev 2.

Harker, Y. D., Blackwood, L. G., and Meachum, T. R. (1995). "Uncertainty Analysis of the SWEPP Drum Assay System for Graphite Content Code 300," INEL-95/0475, Idaho National Engineering Laboratory, Idaho Falls, ID.

Harker, Y. D., Blackwood, L. G., Meachum, T. R., and Yoon, W. Y. (1996). "Uncertainty Analysis of the SWEPP PAN Assay System for Combustible Waste (Content Codes 330 and 336)," INEL-96/0257, Idaho National Engineering Laboratory, Idaho Falls, ID.

Hogg, R. V. and Craig, A. T. (1978). Introduction to Mathematical Statistics, 4th Edition, New York: Macmillan Publishing.

Iman, R. L. and Conover, W. J. (1982). "A Distribution-Free Approach to Inducing Rank Correlation Among Input Variables," Communications in Statistics, B11(3), 311-334.

Iman, R. L. and Helton, J. C. (1985). "A Comparison of Uncertainty and Sensitivity Analysis Techniques for Computer Models." Technical Report SAND84-1461, Sandia Laboratories, Albuquerque, NM.

Iman, R. L. and Shortencarier, M. J. (1984). "A FORTRAN 77 Program and User's Guide for the Generation of Latin Hypercube and Random Samples for Use With Computer Models." NUREG/CR-3624. Sandia National Laboratories, Albuquerque, NM.

Madansky, A. (1988). Prescriptions for Working Statisticians, New York: Springer-Verlag.

Matthews, S. D., Becker, G. K., Marwill, E. S., Miller, G. V. (1993). "SWEPP Assay System Software Requirements Specification," Idaho National Engineering Laboratory Technical Report EGG-RAM10423.

Neter, J. and Wasserman, W. (1974). Applied Linear Statistical Models: Regression, Analysis of Variance, and Experimental Designs, Homewood, IL: Richard D. Irwin, Inc.

U. S. Department of Energy (1995). "Transuranic Waste Characterization Quality Assurance Program Plan, Revision 0," CAO-94-1010, U. S. Department of Energy, Carlsbad Area Office. 
Yoon, W. Y. (1993). "MCNP Modeling and Verification Calculations I," Idaho National Engineering Laboratory Engineering Design File Document EDF-RWMC-609. 


\section{APPENDIX A \\ COMPUTER PROGRAMS FOR SIMULATING PAN ASSAY SYSTEM CALCULATIONS}

A computer application (referred to as SAS PAN) was developed which implements the methodology discussed in Section 7. The application was written using the programming language within the SAS ${ }^{\circledR}$ System, a commercially available integrated software package. The application was initially developed for use on the graphite waste form (content code 300), and was modified prior to use with the combustible waste form (content codes 330 and 336) to implement numerous programmatic modifications. Verification and validation of the computer application are addressed in an INEL internal document (Engineering Design File RWMC-848, Rev. 1).

Applying the SAS PAN application to the glass waste (content codes 440,441 , and 442 ) required only minor modifications from use on the combustible waste. These modifications involved only the waste form specific variables. A complete listing of the computer code used for the glass waste form is provided as Attachment A-A.

\section{Changes to Waste Form Specific Variables}

A brief discussion on the changes in variable values or sources from the combustible waste form to the glass waste form is provided in this section. For a complete discussion on the variable names, values, and sources refer to the next section on variable naming conventions.

Count time (t_c). For the glass waste form, the calibration drum count time varied depending on the content code. (Refer to the discussion in Section 7.2.3). The waste drum simulation count time is fixed for all waste forms processed to date.

Integrals (intrgls). The values used for the glass waste form for the integrals shown in Equation 7.23 differed from those used for the combustible waste form due to differences in the waste forms.

Mass fraction (mf_40_a3). The value used for the mass fraction for the glass waste calibration drum ZPPR sources was based on the average mass fraction for a set of plates discussed in EDF RWMC 779, while the value used for the combustible waste ZPPR sources was based on the mass fraction of only one plate. No changes were made to the mass fraction value used for the calibration drum NAD sources nor to the method used to statistically generate the values used for the waste drum simulations.

Waste drum background coincidence count rate (b_c). The values used for the waste drum background coincidence count rate were statistically generated from a data base of actual daily background drum measurements. For both the glass and combustible waste drum simulations, the values used are calculated within the algorithm program, based on simulated daily background coincidence count rates for the specific waste form. Therefore the actual equation used in the listing for the glass waste form varies from that used for the combustibles.

Waste drum background singles count rate (b_s). The values used for the waste drum background singles count rate were statistically generated from a data base of actual daily background drum measurements. For both the glass and combustible waste drum simulations, the values used are calculated within the algorithm program, based on simulated daily background singles count rates for the specific 
waste form. Therefore the actual equation used in the listing for the glass waste from varies from that used for the combustibles.

\section{Variable Naming Conventions}

Referring to the program listing in Attachment A-A, the variable names used were chosen to follow the naming conventions established in Section 7, where possible. Differences arose between the code variable names and those used in Section 7 when both upper and lower case variables were used in Section 7 . For example, the total accidental coincidence rate is denoted as ' $a$ ' in Section 7, while the singles count rate due to uncorrelated neutrons is denoted as ' $\mathrm{A}$.' In the computer application, ' $\mathrm{A}$ ' is defined as the singles count rate due to uncorrelated neutrons, while 'arate' is defined as the total accidental coincidence rate. Equation numbers are also provided in the computer listing for easy cross-reference to the discussion in Section 7.

For each simulation type, the applicable algorithm program is designed to first define any $S A S^{\circledR}$ macros to be used and then define the input variables and program constants needed, followed by the analysis procedure and algorithm calculations. Input variables and program constants are either read in from external data files or are hard coded. In Attachment A-A, the hard coded program constants shown are applicable to the glass waste forms; modifications to some of these constants are expected for other waste forms.

Initially, separate temporary $S A S^{\circledR}$ data sets are defined for shielded (short gate) and system (long gate) coincidence unit calculations. Prior to the calculation of the moderator index, which is determined from both the net shielded singles rate and net system singles rate, the temporary data sets are combined. From this point in the algorithm programs, one data set is maintained from which the $\mathrm{Pu}$ mass is selected. This data set also contains the majority of the intermediate variable values for both the shielded and system coincidence units and is saved as a permanent $S A S^{\circledR}$ data set to be used as input for further analysis.

As stated above, several SAS ${ }^{\circledR}$ macros are defined at the start of the algorithm program code listing included in Attachment A-A. Three of the macros are used to determine the parameters from the acquisition phase of the PAN passive measurement as discussed in Section 7.2.3. The raw singles count is determined in macro SNGLCNT, while the number of coincidence gates is determined in macro NUMCOIN, and the raw coincidence count is determined in the macro RAWCOIN. The parameters required for the $\mathrm{Pu}$ mass calculations (refer to Section 7.2.4) are calculated in macro PANANAL.

Table A-1 presents the program constants and input variables used for the glass waste forms, with differences in values between shielded or system, and calibration or waste drum simulation identified. Variable references used in Section 7 (i.e., text notation and parameter reference number, where applicable) are identified, along with the variable names used in the computer application, a short description of the parameter, and the value of the constant used in the analysis or the data source for the variable (e.g., PAN system output or statistically generated). 
Table A-1. Program constants and input variables used for glass waste

\begin{tabular}{|c|c|c|c|}
\hline \multicolumn{2}{|c|}{ Parameter Reference } & \multirow[b]{2}{*}{ Parameter Description } & \multirow[b]{2}{*}{ Value/Source $e^{b, c}$} \\
\hline Section $7^{a}$ & Program Code & & \\
\hline \multicolumn{4}{|c|}{$\begin{array}{l}\text { Global Program Constants (i.e., SAS }{ }^{\circledast} \text { macro variables): } \\
\text { [used for shielded or system gates and calibration drum or waste drum simulations] }\end{array}$} \\
\hline$\delta \mathrm{t}$ & delta_t & dead time of detector system & $0.000005 \mathrm{~s}$ \\
\hline$\lambda_{\mathrm{sf}}$ & lamda_sf & $\begin{array}{l}\text { spontaneous fission decay constant } \\
\text { for } \mathrm{Pu}-240\end{array}$ & $1.893 \mathrm{E}-19$ \\
\hline$\Sigma G_{n} n$ & sum_gv & $\begin{array}{l}\text { [from Equation 7.6: for } \mathrm{Pu}-240 \\
\text { spontaneous fission] }\end{array}$ & 4.102 \\
\hline$t_{d}$ & $t \_d$ & gate delay time & $0.000006 \mathrm{~s}$ \\
\hline$v_{\mathrm{av}}$ & v_av & $\begin{array}{l}\text { average number of neutrons } \\
\text { produced per spontaneous fission of } \\
\mathrm{Pu}-240\end{array}$ & 2.16 \\
\hline MF40 $_{\text {wgpu }}$ & $\operatorname{mf} 40 w g p u$ & mass fraction of weapons grade $\mathrm{Pu}$ & 0.0582 \\
\hline (b7) $\mathrm{MF}_{40}$ & $\mathrm{mf} \_40 \_\mathrm{b} 7$ & mass fraction of $\mathrm{Pu}-240$ & 0.0582 \\
\hline \multicolumn{4}{|c|}{$\begin{array}{l}\text { Simulation Specific Program Variables: } \\
\text { [used for shielded or system gates] }\end{array}$} \\
\hline pass mass & pass_mas & measured mass from PAN & $\begin{array}{l}\text { CAL: PAN output } \\
\text { WASTE: } N / R^{\mathrm{e}}\end{array}$ \\
\hline (a1) $t_{c}$ & t_c & count time & $\begin{array}{l}\text { CAL: } 400 \mathrm{~s} \text { for IDC } 442 \\
600 \text { s for DC } 440 \\
\text { WASTE: } 200 \mathrm{~s}\end{array}$ \\
\hline (a2) $\mathrm{M}_{\mathrm{wg}}$ & m_wg & mass of weapons grade PU & $\begin{array}{l}\text { CAL: calculated based on } \\
\text { \# of sources } \\
\text { WASTE: } \text { stat }^{f}\end{array}$ \\
\hline (a3) $\mathrm{MF}_{40}$ & mf_40_a3 & mass fraction of $\mathrm{Pu}-240$ & $\begin{array}{l}\text { CAL: } 0.0528 \text { for NADs } \\
0.0451 \text { for ZPPRs } \\
\text { WASTE: stat }\end{array}$ \\
\hline (b11) AI & $\mathrm{AI}$ & absorber index & $\begin{array}{l}\text { CAL: stat } \\
\text { WASTE: stat }\end{array}$ \\
\hline
\end{tabular}




\begin{tabular}{|c|c|c|c|}
\hline \multicolumn{2}{|c|}{ Parameter Reference } & \multirow[b]{2}{*}{ Parameter Description } & \multirow[b]{2}{*}{ Value/Source $e^{b, c}$} \\
\hline Section $7^{\mathrm{a}}$ & Program Code & & \\
\hline \multicolumn{4}{|c|}{$\begin{array}{l}\text { Gate Specific Program Variables: } \\
\text { [used for calibration drum or waste drum simulations] }\end{array}$} \\
\hline $\mathbf{T}$ & $\begin{array}{l}\text { T_sg } \\
\text { T_lg }\end{array}$ & coincidence gate time & $\begin{array}{l}0.000035-\mathrm{SH} \\
0.00025-\mathrm{SY}\end{array}$ \\
\hline $\begin{array}{l}\int \mathrm{dt} \eta(\mathrm{t}) \\
\int \mathrm{dt} t^{\prime} \eta\left(\mathrm{t}^{\prime}\right)\end{array}$ & intrgls & [Equation 7.23 integration result] & $\begin{array}{l}\text { IDC } 440: \\
0.2853-\mathrm{SH} \\
0.3746-\mathrm{SY} \\
\mathrm{IDC} 441 \text { or } 442: \\
0.2815-\mathrm{SH} \\
0.3786-\mathrm{SY}\end{array}$ \\
\hline \multicolumn{4}{|c|}{ Gate and Simulation Specific Input Parameters ${ }^{\mathrm{g}}$ : } \\
\hline (a12) $\alpha, n$ & alpha_n & $\begin{array}{l}(\alpha, n) \text { singles count rate } \\
\text { (or net singles rate) }\end{array}$ & $\begin{array}{l}\text { CAL: PAN output } \\
\text { WASTE: stat }\end{array}$ \\
\hline (a13) $B_{s}$ & b_s & $\begin{array}{l}\text { waste drum background singles } \\
\text { count rate }\end{array}$ & $\begin{array}{l}\text { CAL: based on b_c_b8 }{ }^{g} \\
\text { WASTE: stat }\end{array}$ \\
\hline (a14) $B_{c}$ & b_c & $\begin{array}{l}\text { waste drum background coincidence } \\
\text { count rate }\end{array}$ & $\begin{array}{l}\text { CAL: based on b_c_b9 } \\
\text { WASTE: stat }\end{array}$ \\
\hline (a15) $\varepsilon$ & $\mathrm{e}$ & detector efficiency & $\begin{array}{l}\text { CAL: MCNP output } \\
\text { WASTE: MCNP output }\end{array}$ \\
\hline (b8) $B_{c}$ & b_c_b8 & daily background singles count rate & $\begin{array}{l}\text { CAL: PAN output } \\
\text { WASTE: stat }\end{array}$ \\
\hline (b9) $\mathrm{B}_{\mathrm{c}}$ & b_c_b9 & $\begin{array}{l}\text { daily background coincidence count } \\
\text { rate }\end{array}$ & $\begin{array}{l}\text { CAL: PAN output } \\
\text { WASTE: stat }\end{array}$ \\
\hline$(\mathrm{b} 10) \sigma_{\mathrm{bc}}$ & er_bcb10 & $\begin{array}{l}\text { daily background coincidence count } \\
\text { rate error }\end{array}$ & $\begin{array}{l}\text { CAL: PAN output } \\
\text { WASTE: stat }\end{array}$ \\
\hline
\end{tabular}

a. The parameter reference number from Section 7 is shown in parentheses, where applicable.

b. CAL: calibration drum simulations

WASTE: waste drum simulations

c. SH: shielded (short) gate

SY: system (long) gate

d. The input parameter is obtained from the PAN system measurement output file.

e. The input parameter is not required for the waste drum simulations.

f. The input parameter is statistically generated.

g. The parameter is calculated within the program and is based on the PAN output variable identified.

h. The input parameter is obtained from the MCNP output. 
Figure A-1. SWEPP Analysis System Implementation for the Glass Waste Form

\section{Verify program constant values}

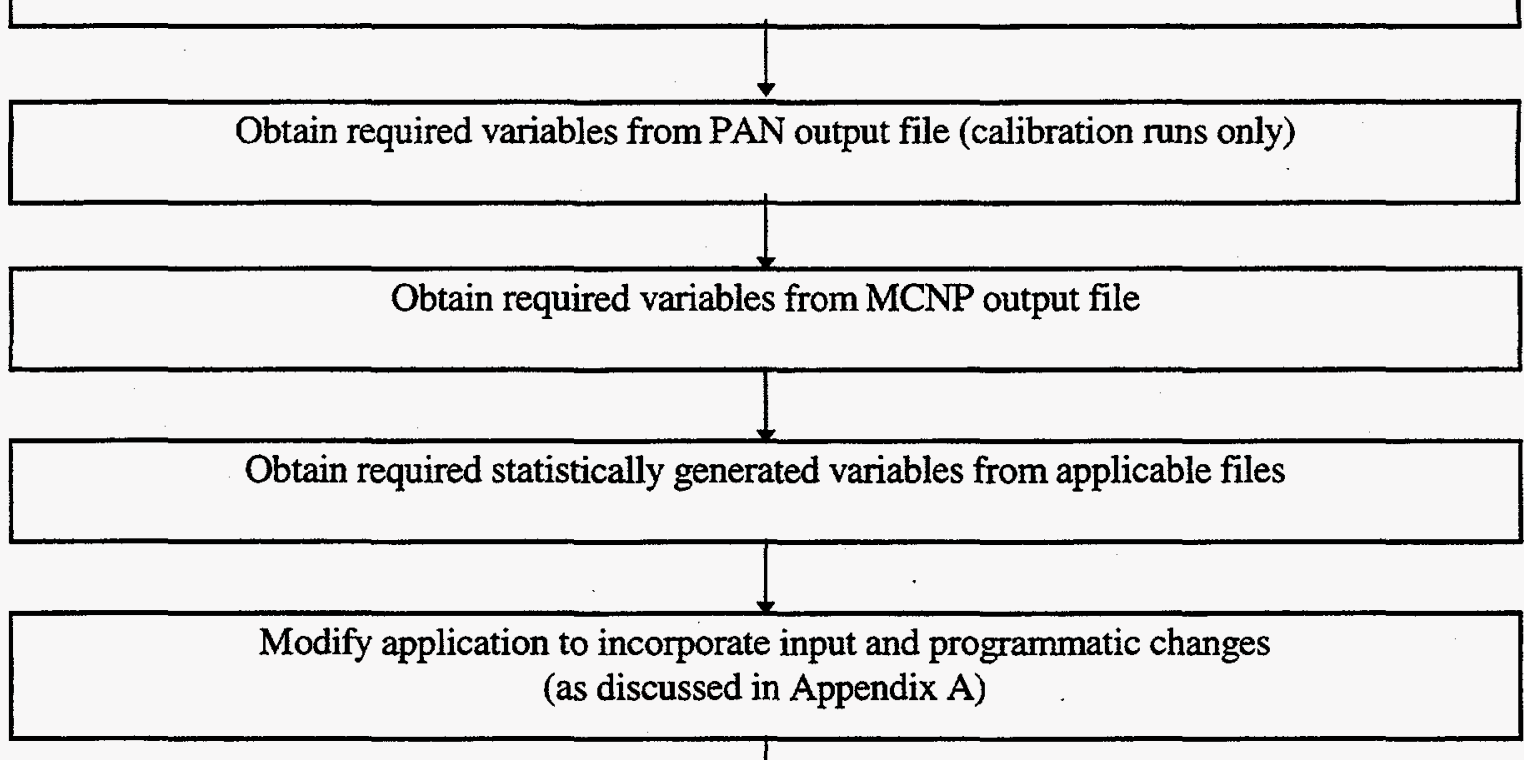

Perform validation checks on modified application if significant changes have been made

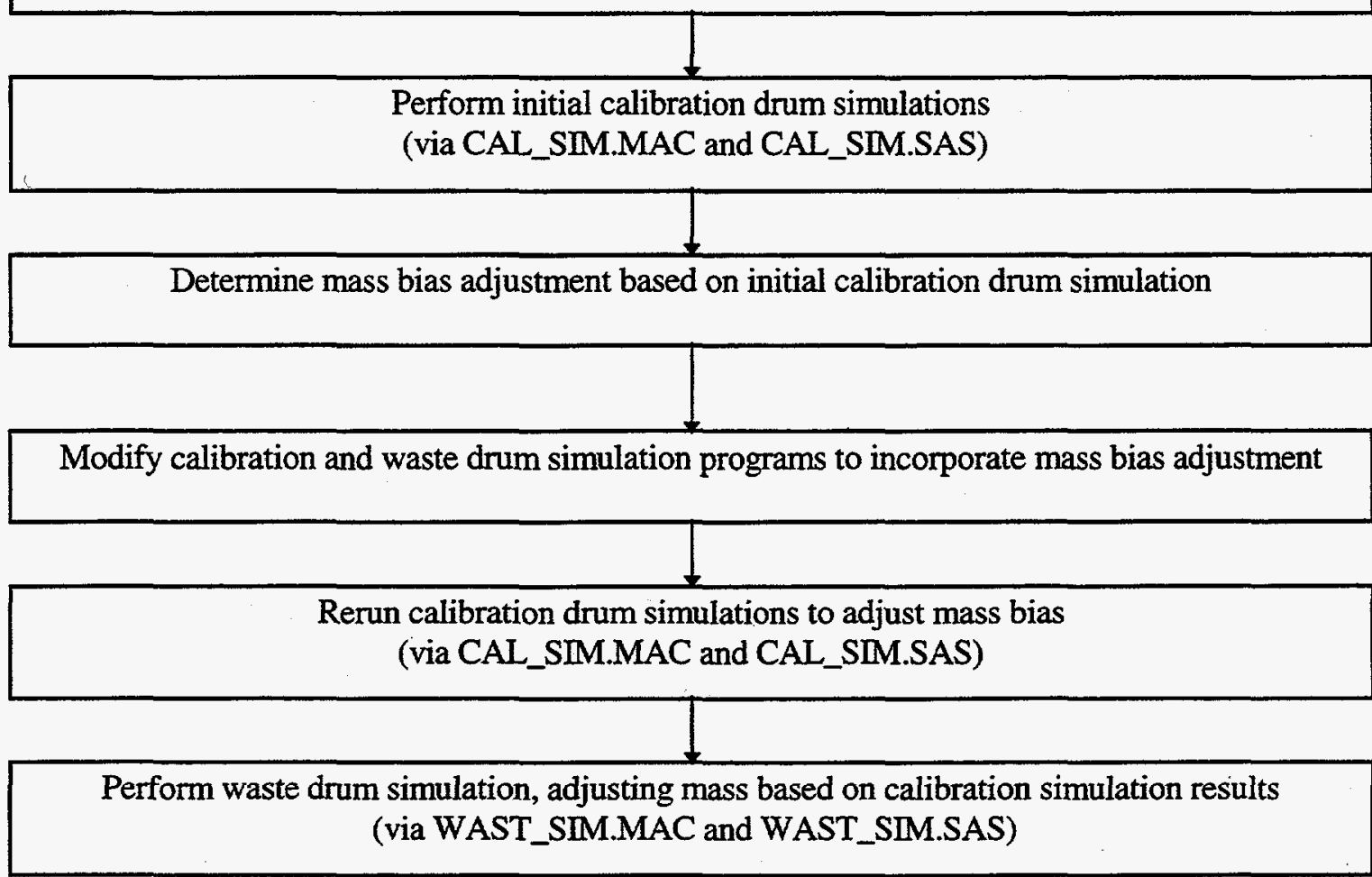


ATTACHMENT A-A

COMPUTER CODE LISTINGS:

1. CAL_SIM.MAC

2. CAL_SIM.SAS

3. WAST_SIM.MAC

4. WAST_SIM.SAS 


\section{CAL_SIM.MAC LISTING}

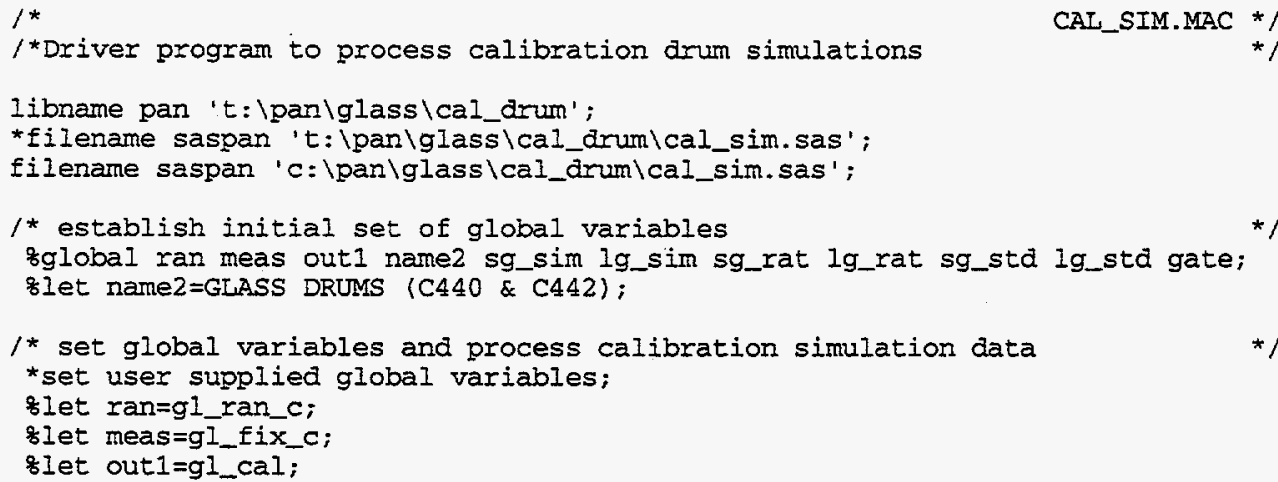




\section{CAL_SIM.SAS LISTING}

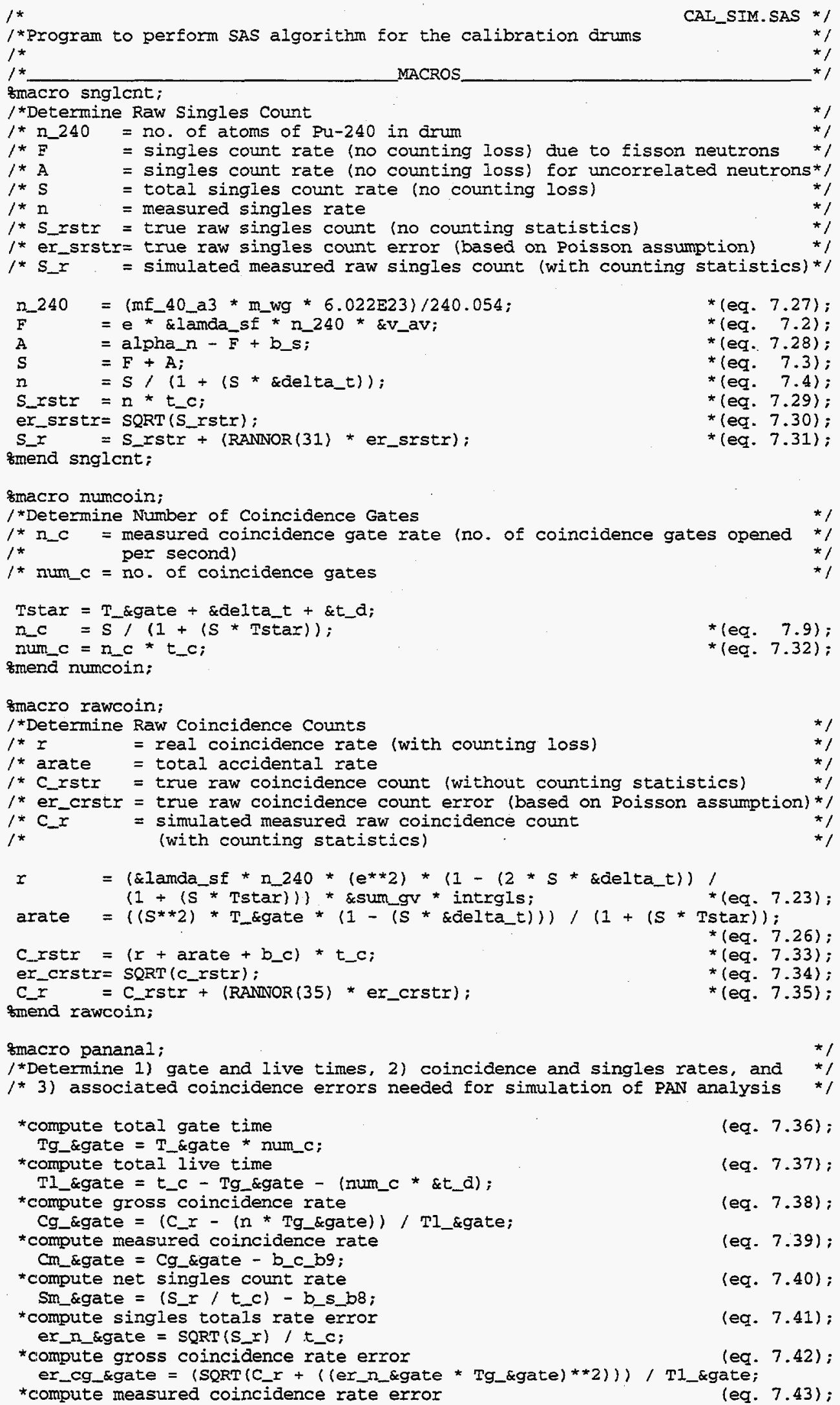


er_cm_\&gate $=\operatorname{SQRT}\left(e r \_c g \_\& g a t e * * 2+\right.$ er_bcb10**2);

smend pananal;

$1 *$ MAIN_PROGRAM

$/$ * Input variables used in SAS Analysis

/* Variables obtained from PAN output

/ *ass_mas = measured mass from PAN

/* alpha_n $=$ total singles count rate

$/ *$ b_s $\quad$ waste drum background singles count rate (a.13)

$f^{*}$ b_c $\quad$ waste drum background coincidence count rate (a.14)

$f^{*} e^{*} \quad$ detector efficiency, on per drum basis (a.15)

$f^{*}$ b_s_b8 = daily background singles count rate (b.8)

$f^{*} \quad$ b_c_b9 = daily background coincidence count rate (b.9)

* er_bcb10= daily background coincidence count rate error (b.10)

$1^{*}$ Variable calculated based on number and type of sources

$j$ * m_wg = mass of Weapons Grade Pu (a.2)

/* Set program constants (units)

$1^{*}$ global constants are -

$1 *$ delta_t $=$ dead time of detector system (s)

$1 *$ lamda_sf= spontaneous fission decay constant for Pu-240 (sec-1)

$1^{*}$ sum_gV = [value given is for Pu-240 spontaneous fission]

$1 * t_{-} a \quad=$ gate delay time (s)

/* $v_{\text {_av }}=$ avg. \# of neutrons produced per spontaneous fission of $\mathrm{Pu}-240$

$1 *$ mf40wgpu= mass fraction of weapons grade PU

$f^{*}$ mf_40_b7 = mass fraction

/*simulation/waste type specific constants are -

$1^{*} t_{-} c \quad=$ count time (s)

$l^{*}$ mf_40_a3 = mass fraction

/* gate specific constants are -

$1^{*}$ T_sg = coincidence gate time for short gate (s)

$1^{*}$ T_lg = coincidence gate time for long gate (s)

$l^{*}$ intrgls $=$ [values given are for the glass content code]

\$global delta_t lamda_sf mf_40_b6 mf40wgpu sum_gv T_d v_av;

slet delta_t $=.000005$;

q let lamda_sf $=1.893 \mathrm{E}-19$;

slet sun $g v=4.102$;

*let t_a $=.000006$;

slet v_av=2.16;

olet mf 40 wgpu $=0.0582$;

slet mf_40_b7 $=0.0582$;

/*PROCESS SHORT GATE (SHIELDED)

/*select needed variables from statistically generated file \& rename

data sg_ran; set pan.\&ran;

keep drum_no idccode ai;

proc sort; by idccode drum_no; run;

/*select needed variables from PAN measurement file data sg_meas; set pan.dmeas;

e =sh_eff;

lpha_n =a_n_sh;

pass_mas $=$ sg_mass;

p_mas_e =sg_mas_e;

b_s_b $\overline{8}=\mathrm{b} \_$sh $r$;

b_c_b9 =b_sh_cr:

er_bcb10=b_sh_cre;

if idccode $=44 \overrightarrow{0}$ then do

b_c $=\left(b \_c \_b 9 * .8868\right)+(\operatorname{RANNOR}(18) * .0131)$

b_s $\quad=\left(b_{\text {LS_b }}\right.$ b8 $\left.* .9704\right)+(\operatorname{RANNNOR}(18) * .106)$;

end:

if idccode $=442$ then do:

b_c $=\left(b \_c \_b 9 * 1.0200\right)+($ RANINOR $(18) * .0131 *$ SQRT $(1.5))$;

b_s $=\left(b \_s \_b 8 * 1.0013\right)+(\operatorname{RANNNOR}(18) * .106 *$ SQRT $(1.5))$

end;

keep idccode drum_no e miwg pass_mas p_mas_e alpha_n mf_40_a3 b_s b_s_b8

b_c_b9 er_bcblo pangate source b_c;

proc sort; by idccode drum_no; 
/*combine input datasets into one input dataset for short gate

data short;

merge sg_ran sg_meas; by idccode drum_no;

set sg_meas;

* define short gate specific constants;

slet gate $=s g$;

T_sg $=.000035$;

if idccode $=440$ then intrgls $=.2853$;

if idccode $=442$ then intrgls $=.2815$;

* set count time based on idccode;

if idccode $=442$ then $t_{-} c=400$;

else t_c $=600$;

/*call macros for short gate data

ssnglent:

onumcoin:

srawcoin;

spananal;

run;

/*PROCESS LONG GATE (SYSTEM)

/*For glass, no statistically generated variables are required, since

$1 *$ absorb has been read in for short gate.

/*select needed variables from PAN measurement file

data Ig_meas; set pan.\&meas;

e =sy_eff;

alphe $n=a$ s sy;

pass_mas=Ig_mass;

p_mas_e $=$ lg_mas_e;

b_s_b8 =b_sy_r;

b_c_b9 =b_sy_cr;

ex_bcb10=b_sy_cre;

if idccode $=440$ then do:

b_c $=($ b_c_b9 *.9187) $+($ RANNOR $(18) * .0521) ;$

b_s $\quad($ b_s_b8 $* .9379)+($ RANNOR $(18) \cdot .257)$;

end;

if idccode $=442$ then do;

b_c $=($ b_c_b9 *1.0173) + (RANNNOR(18)*.0521* SQRT (1.5));

b_s $($ b_s_b8 * 0.9654$)+(\operatorname{RANNOR}(18) * .257$ * SQRT $(1.5))$;

end;

keep idccode drum_no e m_wg pass_mas p_mas_e alpha_n mf_40_a3 b_s_b8

b_s b_c_b9 er_bcb10 source b_c;

proc sort; by idccode drum_no;

data long; set lg_meas;

*define long gate specific constants;

flet gate=lg;

T_L $I g=.000250$;

if idccode $=440$ then intrgis $=.3746$;

if idccode $=442$ then intrgls $=.3786$;

* set count time based on ZPPRS vs. NADs;

if idccode $=442$ then $t \_c=400$;

else t_c $=600$;

$1^{*}$ call macros for long gate data

ssinglcnt;

snumcoin;

srawcoin;

spananal;

run;

/*rename short gate variables by adding "sh" to variable name to distinguish*/ $/$ * Erom long gate (system) variables

data shield; set short;

proc sort; by idccode drum_no;

data shield; set shield;

rename s_rstr=s_rs_sh $s \_r=s \_r \_s h ~ n=n \_s h ~ n \_c=n \_c \_s h$ alpha_n=a_n_sh

num_c=num_c_si $r=r \_s h$ arate=arate_sh c_rstr=c_rs_sh c_r=c_r_sh

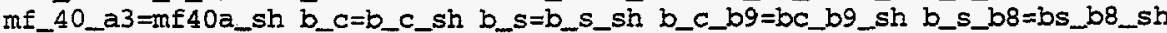
er_bcblo=erbc_sh e=eff_sh $s=s \_s h$ tstar=tstar_sh

run;

intrgls=intgr_sh er_n_sg=er_r_sh Sm_sg=Sm_sh pass_mas=sg_mass p_mas_e=sg_mas_e;

/*rename long gate variables by adding "sy" to variable name to distinguish*/ 
1 * from short gate (shielded) variables

data sys; set long:

proc sort; by idccode drum_no;

data sys; set sys;

rename s_rstr=s_rs_sy s_ras_r_sy n=n_sy n_c=n_c_sy alpha_n=a_n_sy

num_c=num_c_sy $r=r_{\text {__sy }}$ arate=arate_sy c_rstr=c_rs_sy c_r=c_r_sy mf_40_a3=mf40a_sy b_c=b_c_sy b_s=b_s_sy b_c_b9=bc_b9_sy b_s_b8=bs_b8_sy er_bcbl0=erbc_sy e=eff_sy s=s_sy tstar=tstar_sy

drop m wg:

intrgls=intgr_sy er_n_lg=er_n_sy Sm_lg=Sm_sy pass_mas=Ig_mass p_mas_e=1g_mas_e;

run;

1 *COMBINE SHORT AND LONG GATE DATA INTO ONE DATASET

$1^{*}$ compute mass for both shielded and system

data eq44 49; merge shield sys; by idccode;

if pangate $=$ 'Ig' then pass_mas=lg_mass;

else if pangate='sg' then pass_mas=sg_mass;

* compute moderator index.

if $\mathrm{Sm}$ sh $<=10$ then $M I=0.15$

if Smsy $<=0$ then $M I=0$;

if Sm_sh $>10$ and Sm_sy $>0$

then $M I=\left(1-\left(4.4 \overrightarrow{2} *\left(S m \_s h / S m \_s y\right)\right)\right) *(1.04+(0.2924 * \log (A I)))$;

* compute isotopic correction factor

if \&mf_40_b7<0.01 or \&mf_40_b7>0.99 then $C F=1.0$;

(eq. 7.45$)$;

else $C F=$ emf40wgpu / scinf_40_b7;

* compute shielded coincidence correction factor

CF_sh $=\{(0.8092 /(1-\mathrm{MI})\}+0.2337) * \star 2 ;$

(eq. 7.46$)$

* compute system coincidence correction factor

$C F_{-S Y}=\{(0.5967 /(1-M I)\} \div 0.4187) * \star 2 ;$

(eq. 7.47);

* compute Pu mass from shielded coincidence data

(eq. 7.48 );

M_si $=28.1 * C_{1}$ _sh $* C F * C m \_s g$;

* Compute pu mass from system coincidence data

(eq. 7.49);

M_sy $=1.28 * C F \_s y * C F * C m \_l g ;$

/*adjust simulated mass calculations by calibration factor;

data eq50on; set eq44_49;

sh_m_pu=m_sh;

sy_m_pu=m_sy;

*use the following two lines for the initial calibration simulation run;

M_sh = sh_m_pu;

$M s y=s y m$ pu

*use the following two lines for the final calibration simulation run, ;

* adjusting for the mass bias - based on discussion in section 8.2 ;

M sh=(shmpu * 1.195$)-1.671$;

M_sy $=($ sy_m_pu $* 1.040)-.0784$;

* Compute error in Pu mass for shielded coincidence data (eq. 7.50);

er_sh = er_cm_sg * M_sh / Cr_sg;

* compute error in pu mass for system coincidence data run

er_sy $=$ er_cm_lg * M_sy $/ \mathrm{cm}$ lg.

$l^{*}$ select passive plutoniun mass

data pan. \&outl; set eq50on;

if $a b s\left(\left(e r \_c m \_s g / C m \_s g\right)\right)<=a b s($ (er_cm_lg/Cm_lg)) then do; * (eq. 7.52$)$; $\mathrm{M} p u=\mathrm{M} s h$.

er_pu $=$ er_sh;

selgate=' $\mathrm{sg}^{\prime}$;

end;

else do:

M_pu = M_sy;

er_pu = er_sy;

selgate $=$ ' $\mathbf{l g}$ ' ;

end;

*determine short-gate and long-gate to actual mass ratios;

sg_ratio $=$ M_sh/sg_mass;

lg_ratio $=M$ sy $/$ lg_mass :

run:

M_ratio $=$ M_pu/pass_mas; 


\section{WAST_SIM.MAC LISTING}

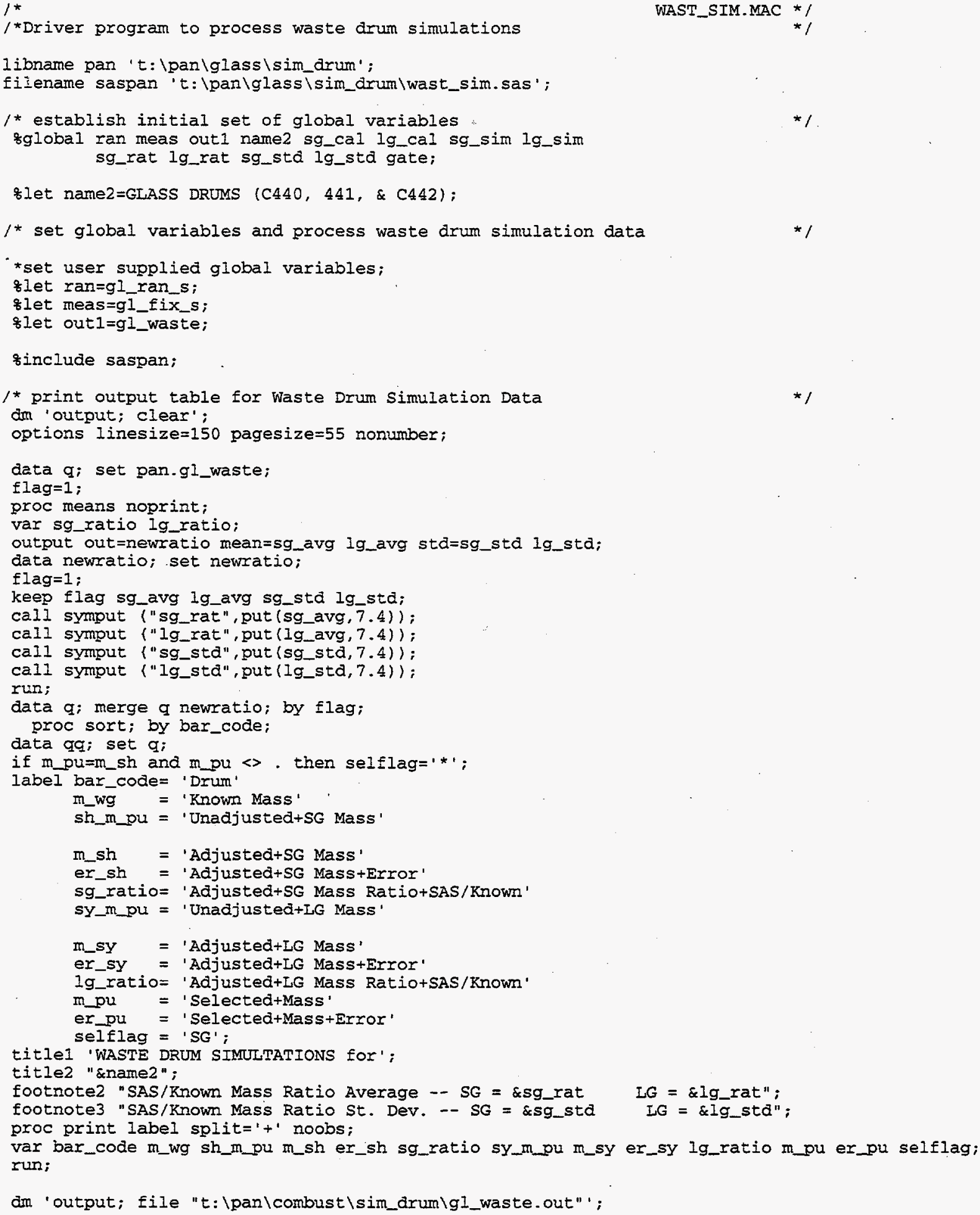




\section{WAST_SIM.SAS LISTING}

$1 *$

/*Program to perform SAS algorithm for the glass waste drums

$1 *$ MACROS

WAST_SIM.SAS * 1

smacro snglent;

$1 *$ Determine Raw Singles Count

$1 * n \_240=$ no. of atoms of Pu-240 in drum

$1 * \mathrm{~F} \quad$ = singles count rate (no counting loss) due to fisson neutrons */

/* A $\quad$ singles count rate (no counting loss) for uncorrelated neutrons*/

$1 * \mathrm{~S} \quad=$ total singles count rate (no counting loss)

$1 * \mathrm{n} \quad=$ measured singles rate

* S_rstr = true raw singles count (no counting statistics)

$f^{*}$ er sxstr = true raw singles count error (based on Poisson assumption) */

$1 * S_{-} r$ simulated measured raw singles count (with counting statistics)*/

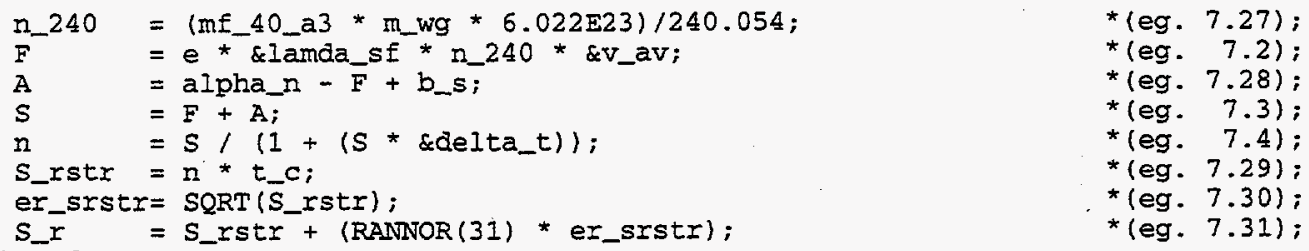

smend snglent:

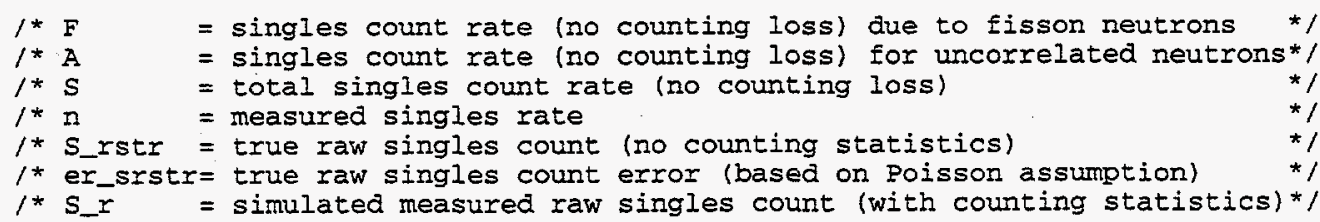

smacro numcoin;

1 *Determine Number of Coincidence Gates

$/^{*}$ Determine Number of Coincidence Gates
$l^{*} \mathrm{n} C \mathrm{~m}=$ measured coincidence gate rate (no. of coincidence gates opened *
per second)

$l^{*}$ num_c $=$ no. of coinciderice gates

Tstar $=$ T_\&gate $+\& d e l t a \_t+\& t \_d ;$

n_c $=S,(1+(S *$ Tstar $))$;

num $c=n+c * t c c$

smend numcoin;

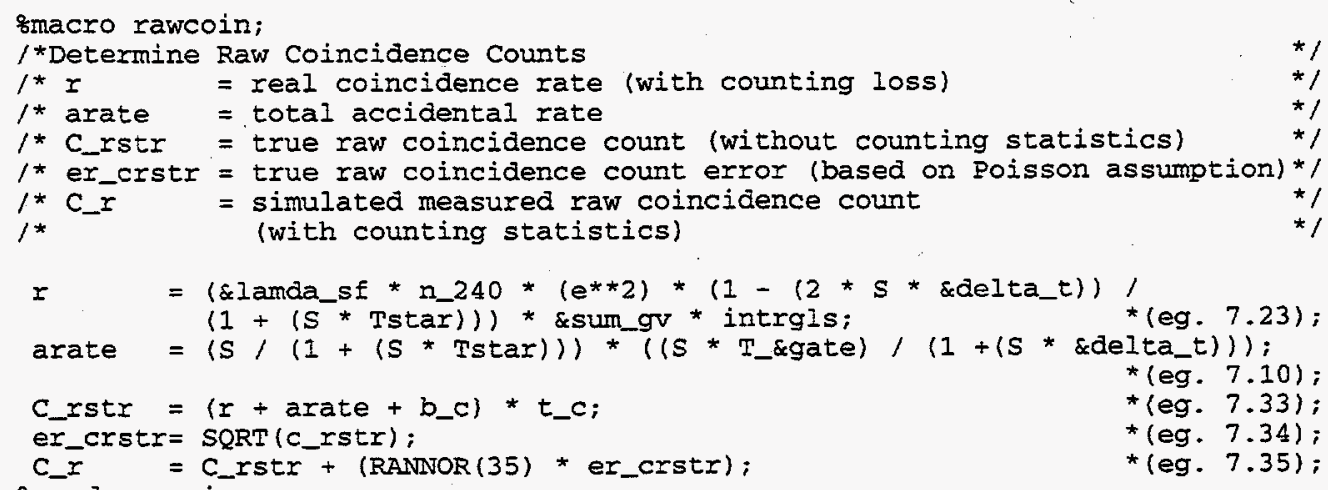

(eg. 7.36);

(eg. 7.37);

(eg. 7.38); 


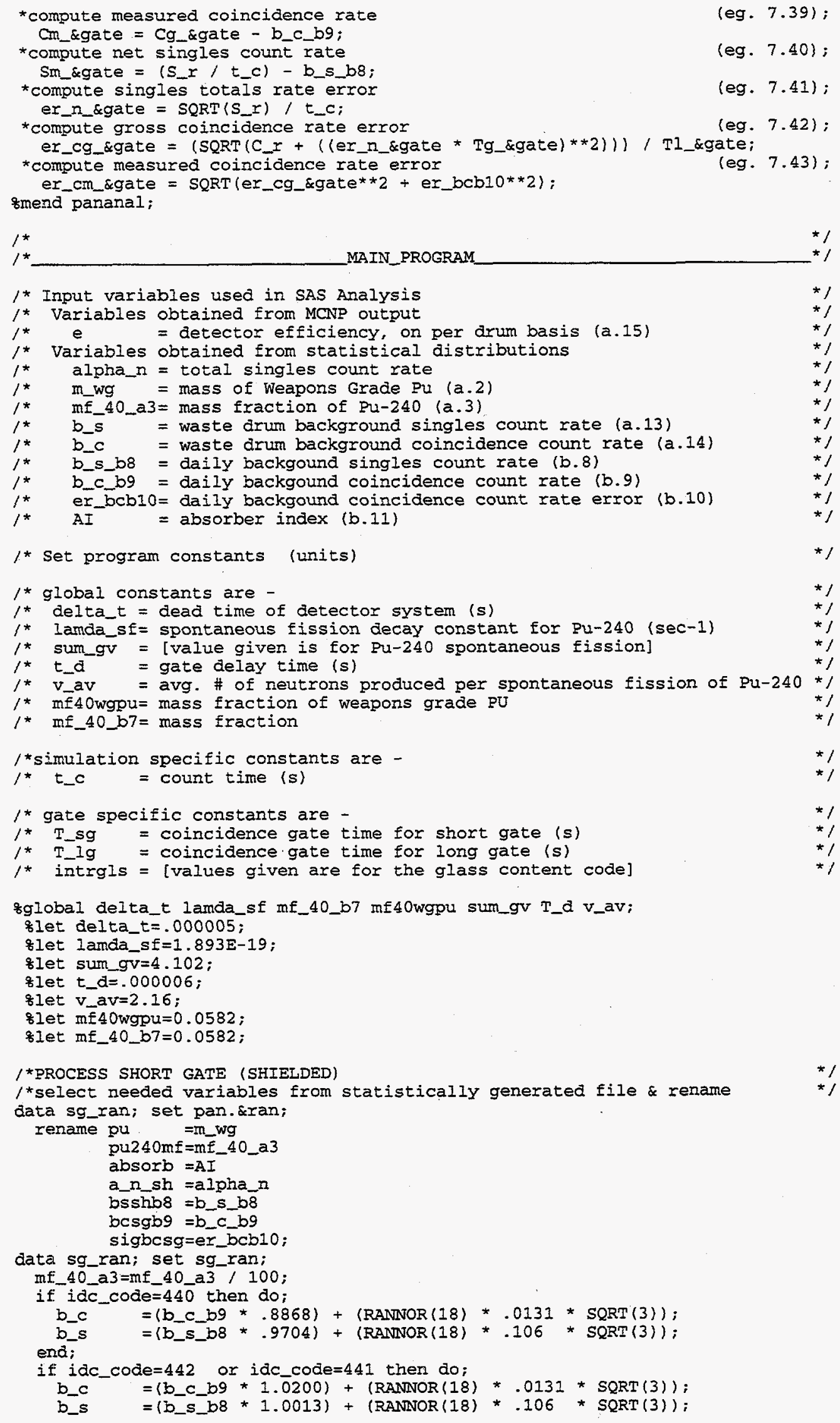


end;

keep order idc_code bar_code m_wg AI mf_40_a3 alpha_n b_s_b8 b_c_b9 er_bcb10 b_c b_s:

proc sort; by order;

$/$ *select needed variables from MCNP file */

data sg_meas; set pan.\&meas;

rename sh_eff=e;

data sg_meas; set sg_meas;

keep e order idc_code bar_code;

proc sort; by order;

/*combine input datasets into one input dataset for short gate *

data short;

merge sg_ran sg_meas; by order;

set sg_meas;

*define short gate specific constants;

flet gate=sg;

T_sg $=.000035$;

if idccode $=440$ then intrgls $=.2853$;

else intrgls $=.2815$;

* set count time;

$t_{-} c=200$;

${ }^{*}$ call macros for short gate data

ssnglont :

snumcoin;

srawcoin:

\&pananal;

run;

/*PROCESS LONG GATE (SYSTEM) */

/*select needed variables from statistically generated file \& rename */ data 1g_ran; set pan.\&ran;

rename pu =m_wg

pu2 $40 \mathrm{mf}=\mathrm{mf}$

absorb =AI

a_n_sy =alpha_n

bssyb8 $=\mathrm{b} s$ s b8

bclgb9 =b_c_b9

sigbclg=er_bcb10

data lg_ran; set lg_ran;

mf_40_a3 =nf_40_a3/ 100 ;

if idc_code $=440$ then do;

b_c $=($ b_c_b9 *.9187) $+($ RANNOR $(18) * .0521 *$ SQRT $(3))$;

b_s $=\left(b_{-}\right.$s_b8 $\left.* .9379\right)+(\operatorname{RANNOR}(18) * .257 *$ SQRT $(3)) ;$

end;

if idc_code $=442$ or idc_code $=441$ then do;

b_c $=\left(b \_c \_b 9 * 1.0173\right)+($ RANNOR $(18) * .0521 *$ SQRT $(3))$;

b_s $\quad=($ b_s_b8 $* 0.9654)+($ RANNOR $(18) * .257 *$ SQRT $(3))$;

end;

keep order idc_code bar_code m_wg AI mf_40_a3 alpha_n b_s_b8 b_c_b9 er_bcb10

$$
\text { b_c b_s; }
$$

proc sort; by order:

/*select needed variables from MCN? file

data lg_meas; set pan.\&meas;

rename sys_eff=e;

data Ig_meas; set 1g_meas;

keep e order idc_code bar_code;

proc sort; by order;

data Iong;

merge Ig_ran 1g_meas; by order;

set lg_meas;

* define long gate specific constants;

slet gate $=19$;

$T \_I g=.000250$;

if idccode $=440$ then intrgls $=.3746$;

else intrgls $=.3786$;

* set count time:

$t_{\text {_c }}=200$; 
ssiglcnt

snumcoin

Srawcoin;

spananal.

run;

/*rename short gate variables by adding "sh" to variable name to distinguish*/ $1^{*}$ from long gate (system) variables

data shield; set short;

proc sort; by order;

data shield; set shield;

rename s_rstras_rs_sh $s \_r=s \_r \_s h ~ n=n \_s h ~ n \_c=n \_c \_s h$ alpha_n=a_n_sh

num_c=num_c_sh $r=r \_s h$ arate=arate_sh c_rstr=c_rs_sh c_r=c_r_sh $\mathrm{mf} 440$ _a3 $=\mathrm{mf} 40 \mathrm{a} \_s h$ b_c er_bcb10=erbc_sh e=eff_sh $s=s_{\text {_tsh }}$ tstar=tstar_sh

run; intrgls=intgr_sh er_n_sg=er_n_sh Sm_sg=sm_sh;

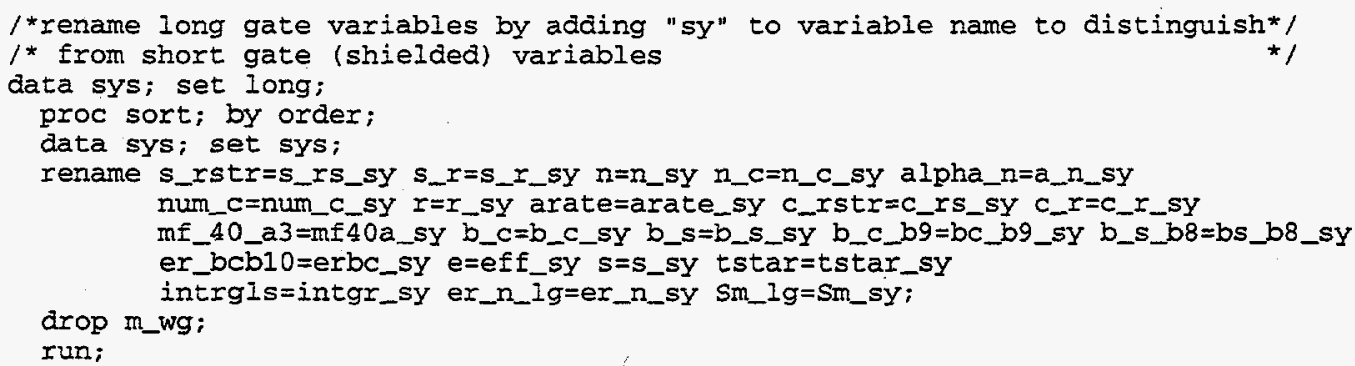

$1 *$ COMBINE SHORT AND LONG GATE DATA INTO ONE DATASET

"compute mass for both shielded and system

data eq44_49; merge shield sys; by order;

* compute moderator index

if $\mathrm{Sm}$ sh $<=10$ then $\mathrm{MI}=0.15$;

if Sm_sy $<=0$ then $M I=0$;

if Sm_sh $>10$ and $S$ Sm_sy $_{2}>0$

then $\mathrm{MI}=\left(1-\left(4.4 \overline{2} *\left(\mathrm{Sm} \_\right.\right.\right.$sh/Sm_sy $\left.\left.)\right)\right) *(1.04+(0.2924 * \log (\mathrm{AI})))$;

* compute isotopic correction factor

if \&mf_40_b7<0.01 or Emf_40_b7>0.99 then $C F=1.0$;

(eg. 7.45$)$

else $C F=\& m f 40$ wgpu $/$ \&mf $4 \overline{0}_{-} b 7$;

* compute shielded coincidence correction factor

$C F \_s h=((0.8092 /(1-\mathrm{MI}))+0.2337) * * 2 ;$

$(e g \cdot 7.46)$

* compute system coincidence correction factor

CF_sy $=\{(0.5967 /(1-\mathrm{MI})\}+0.4187) * * 2 ;$

$(\mathrm{eg} \cdot 7.47)$

* compute pu mass from shielded coincidence data

$(\operatorname{eg} .7 .48) ;$

M_sh $=28.1 * C F \_s h * C F * C m \_s g ;$

* compute Pu mass from system coincidence data

M_sy $=1.28 * C F \_s y * C F * C m \_1 g ;$

(eg. 7.49);

/*adjust simulated mass calculations by calibration factor;

$(\mathrm{eg} \cdot 7.44)$

data eq50on; set eq44_49.

sh_m_pu=m_sh;

sy_m_pu=m_sy

* the following two lines apply the bias adjustment per section 8.2;

M_sh $=($ sh_m_pu * 1.195$)-1.671$;

M_sy $=($ sy_m_pu * 1.040) - .0784;

* compute error in Pu mass for shielded coincidence data

(eg. 7.50$)$

er sh = er cm sg * $\mathrm{s}$ sh / $\mathrm{cm} s g$

* compute error in Pu mass for system coincidence data

(eg. 7.51); run;

r_sy $=$ er_cm_lg * M_sy $/ \mathrm{Cm}$ Ig

1*select passive plutonium mass;

data pan.\&out1; set eq50on;

if abs ( (er_cm_sg/Cm_sg) $<=a b s\left(\left(e r \_c m \_l g / \mathrm{Cm}_{1} I g\right)\right.$ ) then do; $\mathrm{M} p u=\mathrm{M} s \mathrm{sh}$

er_pu = er_sh;

*(eg. 7.52$)$

* (eg. 7.53$)$ 
end;

eise do:

M_pu $=$ M_sy

er_pu = er_sy;

end;

*(eg. 7.54);

*determine short-gate and long-gate to actual mass ratios:

sg_ratio=M_sh/m_wg;

lg_ratio $=\mathrm{M}$ sy $/ \mathrm{m}$ wg;

M_ratio $=$ M_pu $/ m_{\text {_wg }}$;

run; 


\section{APPENDIX B \\ DIFFERENCES BETWEEN THE STATISTICAL MODELING FOR THE GLASS WASTE VS. COMBUSTIBLE WASTE UNCERTAINTY ANALYSES}

For the most part the statistical modeling for the glass waste uncertainty analysis paralleled that reported previously for the combustible waste. For reference purposes, instances where differences occur are listed below. A short rationale for each change is also given. Details of the new methods used for glass waste are given in the appropriate sections elsewhere in this report.

1. For the combustible content code, absorber index values for simulated measurements were assigned based on random sampling from a statistical distribution. The appropriate distribution was determined by analysis of absorber index values for the specific content code in the existing PAN database. The same distribution was used for both calibration drum simulations and waste drum simulations. For the glass content codes, calibration and waste drum simulations no longer use a common sampling distribution. Furthermore, the derivation of the distributions differs from that used previously. Absorber index values for calibration drums were assigned based on a distribution fit to the actual calibration drum absorber index values. Simulated waste drums were assigned absorber indexes equal to the sum of the measured absorber index for the specific drum being modeled plus a random term. The random component reflects the variability in replicate measurements of absorber index values.

2. Previously actual mass values for calibration drums containing ZPPR plates were calculated assuming the same $\mathrm{Pu}$ value $(32.3 \mathrm{~g}$ ) for each plate. For the glass calibration drums, actual $\mathrm{Pu}$ quantities calculated for each individual ZPPR plate in the drum were summed. The calculated individual Pu quantities did not include Pu241, as its contribution to the total $\mathrm{Pu}$ is very small. For calibration drums containing NAD sources, an average value of $1.024 \mathrm{~g}$ was assumed for each NAD. (The average value was determined from an analysis of NADs $\# 206, \# 207$, and $\# 208$ ). This value is more correct than the value of 1.025 used for the combustible calibration drum analysis.

3. Random terms associated with simulated waste drum parameters are no longer generated using Latin hypercube sampling. Simple random sampling is used instead. In general Latin hypercube sampling is only used for a) certain parameters describing matrix information--to insure that full range of matrix configurations is covered, or b) for non-matrix parameters in cases where a correlation structure needs to be imposed on the results. (This is done for convenience as the Latin hypercube sampling program easily handles the correlations.)

4. The method of calculating the standard deviation for zone to zone variability within simulated waste drums was somewhat simplified from that used for combustibles.

5. Some changes in the modeling of $(\alpha, n)$ singles count rates were made to better simulate the $(\alpha, n)$ patterns observed for the glass waste. In particular a mixture distribution was used. 
APPENDIX C
DATA TABLES 
Table C-1. Comparison of actual and simulated calibration PAN results.

\begin{tabular}{|c|c|c|c|c|c|c|c|c|}
\hline $\begin{array}{l}\text { Content } \\
\text { code }\end{array}$ & $\begin{array}{c}\text { Run } \\
\text { number }\end{array}$ & $\begin{array}{l}\text { Total Pu } \\
\text { mass in } \\
\text { drum } \\
\text { (grams) }\end{array}$ & $\begin{array}{l}\text { Actual } \\
\text { PAN short } \\
\text { gate result } \\
\text { (grams) }\end{array}$ & $\begin{array}{l}\text { Simulated } \\
\text { PAN short } \\
\text { gate result } \\
\text { (grams) }\end{array}$ & $\begin{array}{l}\text { Relative \% } \\
\text { error, } \\
\text { simulated vs. } \\
\text { actual short } \\
\text { gate result } \\
\text { (grams) }\end{array}$ & $\begin{array}{c}\text { Actual PAN } \\
\text { long gate } \\
\text { result } \\
\text { (grams) }\end{array}$ & $\begin{array}{c}\text { Simulated } \\
\text { PAN long } \\
\text { gate result } \\
\text { (grams) }\end{array}$ & $\begin{array}{l}\text { Relative \% } \\
\text { error, } \\
\text { simulated vs. } \\
\text { actual long } \\
\text { gate result } \\
\text { (grams) }\end{array}$ \\
\hline 440 & GLASS001 & 128.791 & 74.587 & 80.952 & 8.534 & 72.611 & 74.402 & 2.467 \\
\hline 440 & GLASSO02 & 128.791 & 75.250 & 72.165 & -4.100 & 74.992 & 73.042 & -2.601 \\
\hline 440 & GLASSO03 & 128.791 & 74.045 & 76.641 & 3.505 & 73.182 & 74.217 & 1.414 \\
\hline 440 & GLASS004 & 128.791 & 75.576 & 84.473 & 11.773 & 78.102 & 78.002 & -.128 \\
\hline 440 & GLASS005 & 128.791 & 81.983 & 89.426 & 9.079 & 77.971 & 81.140 & 4.064 \\
\hline 440 & GLASS006 & 128.791 & 75.261 & 80.770 & 7.320 & 76.008 & 76.917 & 1.196 \\
\hline 440 & GLASSO07 & 257.544 & 174.781 & 163.342 & -6.545 & 158.035 & 149.644 & -5.309 \\
\hline 440 & GLASSO08 & 257.544 & 168.795 & 144.323 & -14.498 & 156.791 & 145.860 & -6.972 \\
\hline 440 & GLASS009 & 64.367 & 29.537 & 34.945 & 18.309 & 34.023 & 34.687 & 1.952 \\
\hline 440 & GLASS010 & 64.367 & 45.283 & 46.104 & 1.815 & 47.524 & 44.774 & -5.786 \\
\hline 440 & GLASS011 & 62.934 & 42.731 & 24.487 & -42.695 & 48.626 & 23.663 & -51.338 \\
\hline 440 & GLASS012 & 128.753 & 75.517 & 79.520 & 5.300 & 75.721 & 77.077 & 1.792 \\
\hline 440 & GLASS013 & 32.173 & 17.092 & 13.765 & -19.464 & 17.359 & 16.203 & -6.654 \\
\hline 440 & GLASS014 & 32.173 & 17.902 & 18.982 & 6.030 & 22.006 & 18.116 & -17.676 \\
\hline 440 & GLASS015 & 32.173 & 18.529 & 16.034 & -13.465 & 22.312 & 19.862 & -10.981 \\
\hline 440 & GLASS016 & 32.173 & 24.954 & 21.159 & -15.209 & 23.442 & 21.431 & -8.580 \\
\hline 440 & GLASS017 & 32.173 & 19.409 & 22.687 & 16.890 & 22.392 & 21.535 & -3.829 \\
\hline 440 & GLASS018 & 32.173 & 21.944 & 22.706 & 3.473 & 24.286 & 22.739 & -6.368 \\
\hline 440 & GLASS019] & 160.945 & 88.270 & 101.048 & 14.476 & 84.659 & 90.596 & 7.012 \\
\hline 440 & GLASS020 & 160.945 & 109.757 & 107.918 & -1.675 & 103.057 & 104.836 & 1.727 \\
\hline 440 & GLASS021 & 64.406 & 42.115 & 37.653 & -10.595 & 39.681 & 38.664 & -2.563 \\
\hline 440 & GLASSO22 & 64.406 & 37.819 & 39.253 & 3.792 & 39.061 & 37.661 & -3.584 \\
\hline 440 & GLASS023 & 64.406 & 35.510 & 35.227 & -.799 & 38.904 & 36.810 & -5.381 \\
\hline 440 & GLASS024 & 64.406 & 37.896 & 40.579 & 7.078 & 41.478 & 40.062 & -3.412 \\
\hline 440 & GLASS025 & 64.406 & 35.713 & 39.652 & 11.029 & 39.221 & 39.449 & .582 \\
\hline 440 & GLASS026 & 8.192 & 3.889 & 2.948 & -24.191 & 4.663 & 4.743 & 1.708 \\
\hline 440 & GLASS027 & 8.192 & 3.008 & 3.868 & 28.588 & 4.442 & 5.377 & 21.057 \\
\hline 440 & GLASS028 & 8.192 & 2.719 & 4.242 & 56.043 & 4.311 & 5.362 & 24.389 \\
\hline 440 & GLASSO29 & 8.192 & 3.908 & 4.091 & 4.680 & 4.626 & 5.405 & 16.828 \\
\hline 440 & GLASS030 & 8.192 & 3.263 & 3.514 & 7.695 & 4.614 & 5.489 & 18.964 \\
\hline 440 & GLASS031 & 8.192 & 2.696 & 3.570 & 32.415 & 4.551 & 5.338 & 17.305 \\
\hline 440 & GLASS032 & 4.096 & 2.701 & 1.903 & -29.548 & 2.412 & 2.957 & 22.608 \\
\hline 440 & GLASS033 & 4.096 & 1.062 & 2.439 & 129.759 & 2.242 & 3.146 & 40.343 \\
\hline 440 & GLASS034 & 4.096 & 1.912 & 2.743 & 43.430 & 2.369 & 3.074 & 29.747 \\
\hline 440 & GLASS035 & 4.096 & 2.325 & -.329 & -114.169 & 2.354 & 2.631 & 11.767 \\
\hline 440 & GLASS036 & 4.096 & 1.798 & 2.279 & 26.768 & 1.991 & 3.072 & 54.248 \\
\hline 440 & GLASS037 & 4.096 & .838 & .628 & -25.034 & 2.859 & 2.787 & -2.524 \\
\hline 440 & GLASS038 & 4.096 & 1.636 & 1.008 & -38.371 & 2.565 & 2.885 & 12.468 \\
\hline 440 & GLASS039 & 4.096 & .785 & .685 & -12.703 & 2.511 & 2.810 & 11.887 \\
\hline 440 & GLASSO40 & 4.096 & 3.753 & 2.290 & -38.974 & 2.544 & 3.111 & 22.296 \\
\hline 440 & GLASSO41 & 4.096 & .845 & 1.304 & 54.294 & 2.750 & 2.934 & 6.690 \\
\hline
\end{tabular}




\begin{tabular}{|c|c|c|c|c|c|c|c|c|}
\hline $\begin{array}{c}\text { Content } \\
\text { code }\end{array}$ & $\begin{array}{c}\text { Run } \\
\text { number }\end{array}$ & $\begin{array}{l}\text { Total Pu } \\
\text { mass in } \\
\text { drum } \\
\text { (grams) }\end{array}$ & $\begin{array}{c}\text { Actual } \\
\text { PAN short } \\
\text { gate result } \\
\text { (grams) }\end{array}$ & $\begin{array}{l}\text { Simulated } \\
\text { PAN short } \\
\text { gate result } \\
\text { (grams) }\end{array}$ & $\begin{array}{c}\text { Relative \% } \\
\text { error, } \\
\text { simulated vs. } \\
\text { actual short } \\
\text { gate result } \\
\text { (grams) }\end{array}$ & $\begin{array}{c}\text { Actual PAN } \\
\text { long gate } \\
\text { result } \\
\text { (grams) }\end{array}$ & $\begin{array}{c}\text { Simulated } \\
\text { PAN long } \\
\text { gate result } \\
\text { (grams) }\end{array}$ & $\begin{array}{l}\text { Relative \% } \\
\text { error, } \\
\text { simulated vs. } \\
\text { actual long } \\
\text { gate result } \\
\text { (grams) }\end{array}$ \\
\hline 440 & GLASSO42 & 4.096 & .671 & .678 & 1.096 & 2.590 & 2.827 & 9.122 \\
\hline 440 & GLASS043 & 2.048 & -.115 & -.834 & 624.794 & 1.303 & 1.382 & 6.083 \\
\hline 440 & GLASS044 & 2.048 & .936 & -.287 & -130.623 & 1.161 & 1.495 & 28.712 \\
\hline 440 & GLASSO45 & 2.048 & 1.133 & -2.005 & -276.938 & 1.406 & 1.238 & -11.924 \\
\hline 442 & RASC01 & 12.288 & 6.600 & 5.472 & -17.091 & 8.234 & 8.966 & 8.899 \\
\hline 442 & RASC02 & 12.288 & 7.135 & 5.657 & -20.719 & 7.924 & 8.465 & 6.828 \\
\hline 442 & RASC03 & 12.288 & 6.813 & 7.903 & 16.003 & 8.274 & 9.308 & 12.501 \\
\hline 442 & RASC04 & 12.288 & 7.655 & 8.474 & 10.707 & 8.090 & 9.011 & 11.387 \\
\hline 442 & RASC05 & 10.240 & 7.448 & 4.650 & -37.571 & 6.530 & 7.103 & 8.776 \\
\hline 442 & RASC06 & 10.240 & 6.364 & 5.419 & -14.861 & 6.344 & 7.078 & 11.577 \\
\hline 442 & RASC07 & 10.240 & 5.647 & 6.652 & 17.783 & 6.791 & 7.601 & 11.925 \\
\hline 442 & RASC08 & 10.240 & 6.574 & 6.261 & -4.750 & 6.699 & 7.516 & 12.200 \\
\hline 442 & RASC09 & 10.240 & 7.263 & 6.462 & -11.026 & 6.732 & 7.375 & 9.549 \\
\hline 442 & RASC 10 & 10.240 & 8.462 & 5.074 & -40.034 & 6.973 & 7.418 & 6.393 \\
\hline 442 & RASC11 & 9.216 & 5.325 & 4.510 & -15.297 & 5.849 & 6.353 & 8.617 \\
\hline 442 & RASC12 & 8.192 & 3.875 & 5.767 & 48.810 & 4.588 & 5.943 & 29.522 \\
\hline 442 & RASC13 & 7.168 & 3.845 & 5.446 & 41.622 & 4.146 & 5.257 & 26.798 \\
\hline 442 & RASC14 & 6.144 & 2.076 & 1.674 & -19.372 & 3.509 & 3.983 & 13.516 \\
\hline 442 & RASC15 & 5.120 & 2.682 & 1.446 & -46.087 & 2.837 & 3.163 & 11.502 \\
\hline 442 & RASC16 & 4.096 & 3.978 & 1.710 & -57.023 & 2.713 & 3.303 & 21.763 \\
\hline 442 & RASC 17 & 3.072 & 1.705 & 2.124 & 24.594 & 2.200 & 2.627 & 19.424 \\
\hline 442 & RASC18 & 3.072 & 1.486 & -.562 & -137.835 & 2.307 & 2.303 & -.154 \\
\hline 442 & RASC19 & 3.072 & 1.775 & -.830 & -146.742 & 2.381 & 2.567 & 7.788 \\
\hline 442 & RASC20 & 4.096 & 2.610 & 1.000 & -61.710 & 2.860 & 3.023 & 5.700 \\
\hline 442 & RASC21 & 6.144 & 2.081 & 2.721 & 30.769 & 4.060 & 4.895 & 20.577 \\
\hline 442 & RASC22 & 193.100 & 131.971 & 121.932 & -7.607 & 117.920 & 124.567 & 5.637 \\
\hline 442 & RASC23 & 160.950 & 98.102 & 108.277 & 10.372 & 98.718 & 106.680 & 8.065 \\
\hline 442 & RASC24 & 128.700 & 79.081 & 91.610 & 15.844 & 78.545 & 86.666 & 10.339 \\
\hline 442 & RASC25 & 96.560 & 56.212 & 65.958 & 17.339 & 57.561 & 62.133 & 7.944 \\
\hline 442 & RASC26 & 32.190 & 18.209 & 21.190 & 16.369 & 19.816 & 20.746 & 4.692 \\
\hline 442 & RASC27 & 64.370 & 45.331 & 45.427 & .212 & 44.559 & 42.873 & -3.783 \\
\hline
\end{tabular}


Table C-2. PAN system measurement results for the simulated glass waste drums.

\begin{tabular}{|c|c|c|c|c|}
\hline Drum number & $\begin{array}{l}\text { True Pu mass } \\
\text { (grams) }\end{array}$ & $\begin{array}{l}\text { PAN Pu Mass } \\
\text { (grams) }\end{array}$ & Relative \% error & $\begin{array}{l}\text { Short/long gate } \\
\text { mass selected }^{1}\end{array}$ \\
\hline 1 & 1 & .4 & -55.0 & \\
\hline 2 & 1 & 1.1 & 8.0 & \\
\hline 3 & 1 & 1.0 & 3.8 & \\
\hline 4 & 1 & 1.0 & -4.8 & \\
\hline 5 & 1 & .2 & -80.5 & \\
\hline 6 & 1 & 1.2 & 16.4 & \\
\hline 7 & 1 & 2.5 & 151.0 & \\
\hline 8 & 1 & -3.0 & -396.5 & * \\
\hline 9 & 1 & .3 & -70.3 & \\
\hline 10 & 1 & -3.8 & -481.0 & * \\
\hline 11 & 3 & 2.3 & -24.3 & \\
\hline 12 & 3 & 1.8 & -41.6 & \\
\hline 13 & 3 & .8 & -73.0 & \\
\hline 14 & 3 & 2.8 & -6.8 & \\
\hline 15 & 3 & 2.4 & -20.2 & \\
\hline 16 & 3 & 1.8 & -40.9 & \\
\hline 17 & 3 & 2.5 & -15.8 & \\
\hline 18 & 3 & 1.3 & -57.8 & \\
\hline 19 & 3 & 2.3 & -23.1 & \\
\hline 20 & 3 & 4.3 & 42.6 & \\
\hline 21 & 5 & 2.7 & -45.4 & \\
\hline 22 & 5 & 3.6 & -27.4 & \\
\hline 23 & 5 & 3.7 & -25.5 & \\
\hline 24 & 5 & 5.7 & 13.2 & \\
\hline 25 & 5 & 3.4 & -32.2 & \\
\hline 26 & 5 & 4.0 & -20.6 & \\
\hline 27 & 5 & 2.9 & -42.0 & \\
\hline 28 & 5 & 5.3 & 6.6 & \\
\hline 29 & 5 & 2.4 & -52.0 & \\
\hline 30 & 5 & 4.8 & -4.9 & \\
\hline 31 & 10 & 13.6 & 35.5 & \\
\hline 32 & 10 & 9.6 & -3.7 & \\
\hline 33 & 10 & 9.0 & -10.5 & \\
\hline 34 & 10 & 8.7 & -12.8 & \\
\hline 35 & 10 & 7.3 & -27.0 & \\
\hline 36 & 10 & 6.5 & -35.3 & \\
\hline 37 & 10 & 8.5 & -14.5 & \\
\hline 38 & 10 & 9.5 & -5.2 & \\
\hline 39 & 10 & -6.5 & -164.7 & * \\
\hline 40 & 10 & 10.4 & 3.5 & \\
\hline 41 & 20 & 14.0 & -29.8 & \\
\hline 42 & 20 & 11.9 & -40.3 & \\
\hline 43 & 20 & 17.8 & -11.2 & \\
\hline 44 & 20 & 11.0 & -44.9 & \\
\hline 45 & 20 & 5.0 & -74.8 & \\
\hline 46 & 20 & 8.4 & -58.1 & \\
\hline 47 & 20 & 21.2 & 5.8 & \\
\hline 48 & 20 & 16.1 & -19.7 & \\
\hline 49 & 20 & 17.0 & -15.2 & \\
\hline 50 & 20 & 12.3 & -38.3 & \\
\hline 51 & 30 & 32.5 & 8.4 & \\
\hline 52 & 30 & 27.8 & -7.5 & \\
\hline 53 & 30 & 23.6 & -21.2 & \\
\hline
\end{tabular}




\begin{tabular}{|c|c|c|c|c|}
\hline Drum number & $\begin{array}{l}\text { True Pu mass } \\
\text { (grams) }\end{array}$ & $\begin{array}{l}\text { PAN Pu Mass } \\
\text { (grams) }\end{array}$ & Relative \% error & $\begin{array}{l}\text { Short/long gate } \\
\text { mass selected }^{1}\end{array}$ \\
\hline 54 & 30 & 25.0 & -16.8 & \\
\hline 55 & 30 & 19.5 & -35.0 & \\
\hline 56 & 30 & 20.2 & -32.8 & \\
\hline 57 & 30 & 30.0 & .0 & \\
\hline 58 & 30 & 25.2 & -15.9 & \\
\hline 59 & 30 & 18.2 & -39.3 & \\
\hline 60 & 30 & 18.8 & -37.5 & \\
\hline 61 & 40 & 24.2 & -39.4 & \\
\hline 62 & 40 & 32.8 & -17.9 & \\
\hline 63 & 40 & 38.7 & -3.3 & \\
\hline 64 & 40 & 41.2 & 3.0 & \\
\hline 65 & 40 & 39.7 & -.7 & \\
\hline 66 & 40 & 25.9 & -35.3 & \\
\hline 67 & 40 & 38.2 & -4.6 & \\
\hline 68 & 40 & 23.4 & -41.5 & \\
\hline 69 & 40 & 33.3 & -16.8 & \\
\hline 70 & 40 & 29.4 & -26.6 & \\
\hline 71 & 60 & 69.4 & 15.6 & \\
\hline 72 & 60 & 39.7 & -33.8 & \\
\hline 73 & 60 & 35.8 & -40.4 & \\
\hline 74 & 60 & 49.5 & -17.6 & * \\
\hline 75 & 60 & 42.9 & -28.4 & \\
\hline 76 & 60 & 36.4 & -39.4 & * \\
\hline 77 & 60 & 49.6 & -17.3 & \\
\hline 78 & 60 & 38.5 & -35.8 & \\
\hline 79 & 60 & 47.8 & -20.4 & * \\
\hline 80 & 60 & 40.9 & -31.9 & \\
\hline 81 & 80 & 71.0 & -11.2 & * \\
\hline 82 & 80 & 29.2 & .63 .6 & \\
\hline 83 & 80 & 34.4 & -57.0 & \\
\hline 84 & 80 & 67.8 & -15.3 & \\
\hline 85 & 80 & 122.9 & 53.6 & * \\
\hline 86 & 80 & 38.9 & -51.4 & \\
\hline 87 & 80 & 67.0 & -16.3 & \\
\hline 88 & 80 & 67.0 & -16.2 & \\
\hline 89 & 80 & 57.7 & -27.9 & \\
\hline 90 & 80 & 49.0 & -38.8 & \\
\hline 91 & 100 & 40.0 & -60.0 & \\
\hline 92 & 100 & 133.3 & 33.3 & * \\
\hline 93 & 100 & 142.4 & 42.4 & * \\
\hline 94 & 100 & 45.2 & -54.8 & \\
\hline 95 & 100 & 45.5 & -54.5 & \\
\hline 96 & 100 & 82.6 & -17.4 & \\
\hline 97 & 100 & 95.1 & -4.9 & \\
\hline 98 & 100 & 67.8 & -32.2 & \\
\hline 99 & 100 & 68.4 & -31.6 & \\
\hline 100 & 100 & 65.4 & -34.6 & \\
\hline
\end{tabular}

1. Short gate coincidence mass chosen as PAN Pu mass. 